Florida International University

FIU Digital Commons

$5-18-2020$

\title{
Systematic Comparison of Pre-Treatment Parameters in Forensic Hair Analysis and Investigating Hair-drug Interactions
}

Jennett Chenevert Aijala

Florida International Universty, jchen076@fiu.edu

Follow this and additional works at: https://digitalcommons.fiu.edu/etd

Part of the Analytical Chemistry Commons, and the Other Chemistry Commons

\section{Recommended Citation}

Aijala, Jennett Chenevert, "Systematic Comparison of Pre-Treatment Parameters in Forensic Hair Analysis and Investigating Hair-drug Interactions" (2020). FIU Electronic Theses and Dissertations. 4521.

https://digitalcommons.fiu.edu/etd/4521

This work is brought to you for free and open access by the University Graduate School at FIU Digital Commons. It has been accepted for inclusion in FIU Electronic Theses and Dissertations by an authorized administrator of FIU Digital Commons. For more information, please contact dcc@fiu.edu. 


\title{
FLORIDA INTERNATIONAL UNIVERSITY \\ Miami, Florida
}

\section{SYSTEMATIC COMPARISON OF PRE-TREATMENT PARAMETERS IN FORENSIC HAIR ANALYSIS AND INVESTIGATING HAIR-DRUG INTERACTIONS}

\author{
A dissertation submitted in partial fulfillment of \\ the requirements for the degree of \\ DOCTOR OF PHILOSOPHY \\ in \\ CHEMISTRY \\ by \\ Jennett Chenevert Aijala
}

2020 
To: Dean Michael R. Heithaus

College of Arts, Sciences and Education

This dissertation, written by Jennett Chenevert Aijala, and entitled Systemic Comparison of Pre-treatment Parameters in Forensic Hair Analysis and Investigating Hair-Drug Interactions, having been approved in respect to style and intellectual content, is referred to you for judgment.

We have read this dissertation and recommend that it be approved.

$\begin{array}{r}\hline \text { John Berry } \\ \hline \text { Bruce McCord } \\ \hline \text { Kevin O'Shea } \\ \hline \text { Deodutta Roy } \\ \hline \text { Anthony P. DeCaprio, Major Professor }\end{array}$

Date of Defense: May 18, 2020

The dissertation of Jennett Chenevert Aijala is approved.

Dean Michael R. Heithaus College of Arts, Sciences and Education

Andrés G. Gil Vice President for Research and Economic Development and Dean of the University Graduate School

Florida International University, 2020 


\section{(C) Copyright 2020 by Jennett Chenevert Aijala}

All rights reserved by the author with the exception of Figures 1, 2, 3, 4, 13, 15, 16, 17,

18, and 19, Appendix 1, and Tables 1, 22, and 23. These figures, tables, and appendices have been included with the permission of the respective publishers. 


\section{DEDICATION}

This dissertation is dedicated to the three groups of people my name is comprised of: my loving and supportive grandmother, Margaret Hawes, née Jennett, my family, the Chenevert's, and to my in-law's, the Aijala's. Without your love and support, I would not be the human I am today. 


\section{ACKNOWLEDGMENTS}

First, I would like to acknowledge my Principal Investigator, Dr. Anthony P. DeCaprio; with your guidance, I have developed stronger research, writing, and presentation skills. Next, I would like to thank my committee members: Dr. John Berry, Dr. Bruce McCord, Dr. Kevin O’Shea, and Dr. Deodutta Roy. Thank you for consistently providing constructive feedback and words of advice. All of you have helped me to become a better scientist throughout my graduate school experience, and I am grateful.

I would like to thank the National Institute of Justice (NIJ) and the Center for Advanced Research in Forensic Science (CARFS) for funding provided to my research and funding my travel to national conferences. I would also like to thank the Florida International University Department of Chemistry for providing funding through a teaching assistantship prior to receiving NIJ funding.

Those who have been around me in the last five years have heard me say jokingly, "Grad school is sad school". While there was sometimes more truth to this statement than other times, there are a few individuals that I must acknowledge for making me a better scientist and a better human throughout this process. First, I owe the biggest THANK YOU I can give to Chiara Deriu. Thank you for helping me revise and edit this document, and all the others; thank you for studying with me at the kitchen table until it was much too late; thank you for the morale boosts when graduate school got me down; thank you for being an incredible roommate and an even more incredible friend. Chiara, I respect you deeply and I am grateful to have you in my life.

Next, I want to thank all of my lab-mates, past and present, for your contributions to my development. Dr. Melanie Eckberg and Dr. R. Allen Gilliland, thank you for having 
patience with me. Dr. Ashley Kimble, thank you for always being the person I can go to for advice in the lab. I enjoyed the time we all got to work together, and I am grateful to you all for your support and guidance as I transitioned into graduate school, and as I became one of the more senior members of the group. William Morrison IV, thank you for your direct and constructive criticism on my work and on my NFL affiliations. Brianna Spear, thank you for taking an interest in this work and taking on the task of moving this research forward; I trust that you will make this project your own, and I know you will do great things. Thank you Dr. Anamary Tarifa for your support in the Forensic Analytical Toxicology Facility. Ludmyla Tavares, Rebecca Smith, Meena Swaminathan, and Leo Maya, thank you for listening to my presentations and giving thoughtful feedback.

Finally, I want to thank my friends and family; without your love and support, I would not have been able to accomplish all that I have. Thank you to my parents, and I mean all of you, for helping to guide me through life to make good decisions and follow through on my commitments. Thank you to my younger brothers, Vinny and Alex, for always inspiring me to be my best for you. Thank you, Marc, for always being a patient listener, a supporting husband, and the love of my life. Thank you, Erin, for being one of my people, for sharing the joys of your life with me, and for inspiring me to be the best version of myself. Thank you, Quentin, for being such a down-to-earth presence in my life throughout this process. Thank you, Michelle, for always being equally down to study or go dancing. Thank you, Jeff and Will, for watching the Patriots games with me.

I honestly do not have enough words to express the amount of gratitude I feel because of the opportunities I have been afforded and the wonderful humans who have helped me accomplish my goals. 


\begin{abstract}
OF THE DISSERTATION
SYSTEMATIC COMPARISON OF PRE-TREATMENT PARAMETERS IN

FORENSIC HAIR ANALYSIS AND INVESTIGATING HAIR-DRUG
\end{abstract}

INTERACTIONS

\author{
by \\ Jennett Chenevert Aijala \\ Florida International University, 2020 \\ Miami, Florida \\ Anthony P. DeCaprio, Major Professor
}

Forensic toxicological analysis for the purposes of protecting human health and supporting criminal justice activities can be achieved, in part, by means of analysis of human head hair for detection of abused substances. Hair is considered an alternative sample matrix (compared to blood and urine) because of persistent interpretive challenges caused by a lack of standardization in analytical approaches and poor understanding of the biases resulting from physicochemical interactions between major hair components (e.g., melanin) and drugs of abuse. Some of the uncertainty regarding optimal techniques for the pre-treatment of hair specimens results from the unknown nature of the interactions between drugs and hair components, in particular melanin. Thus, the second focus of this work was to study these interactions with select drugs and melanin by UV/Visible spectroscopy. Another source of uncertainty is attributed to the lack of standardization in the analytical approach to hair testing. As a complex solid sample matrix, hair requires pretreatment measures including decontamination, homogenization, and extraction to remove drug from the hair components to allow for analysis. Optimizing these pre-treatment 
parameters is essential for accurate toxicological analysis of this matrix. Thus, a major focus of this work was to conduct a comprehensive comparison of the efficacy of decontamination and extraction variables by means of statistical design of experiments (DoE).

Major findings of this work were a minimum of four consecutive washes were necessary for the decontamination of hair and solvent swelling of the matrix resulted in the highest extraction of the five analytes under study. The other major conclusion of this work was the interaction between eumelanin and drugs is a result of ionic interactions and van der Waals forces. Additionally, association constants between drugs and eumelanin were determined to provide additional insight into the strength of interactions between drugs with different physicochemical properties and eumelanin.

These findings will impact the forensic science community by contributing to a body of research aimed at determining the most effective method(s) for decontaminating hair samples for forensic analysis, the subsequent isolation of drugs from hair, and by investigating the interactions between melanin and select drugs of abuse. 
CHAPTER

PAGE

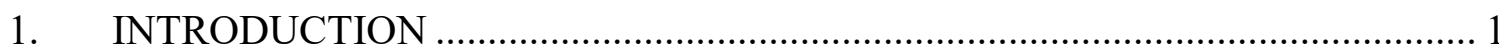

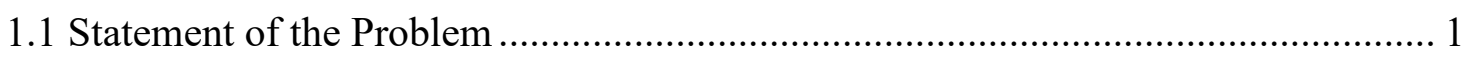

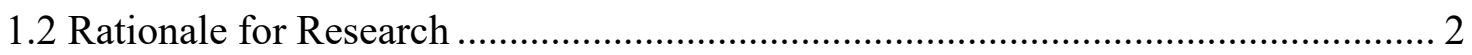

1.2.1 Task 1 - Preparation of incorporated/entrapped, single analyte hair reference material (HRM). ..................................................................................... 3

1.2.2 Task 2 - Systematic comparison of hair decontamination parameters via $2^{4}$

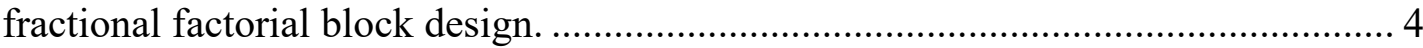

1.2.3 Task 3 - Systematic comparison of extraction parameters in forensic hair analysis via a $2^{3}$ full factorial design.

1.2.4 Task 4 - Probing physicochemical interactions between drugs of abuse and eumelanin.

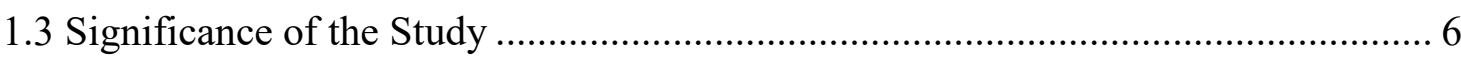

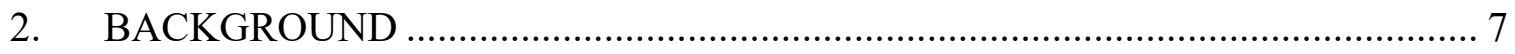

2.1 Cellular and molecular components of hair fibers ................................................ 7

2.2 Incorporation mechanisms of drugs of abuse into hair ........................................ 13

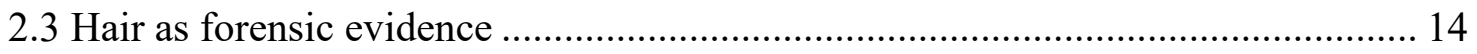

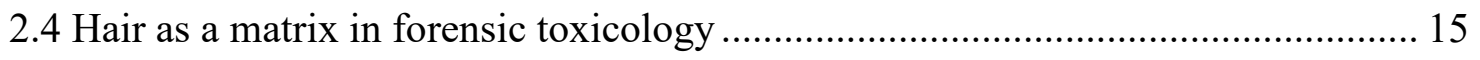

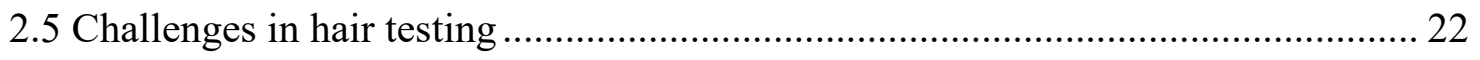

2.6 Introduction to Statistical Design of Experiments …………............................... 26

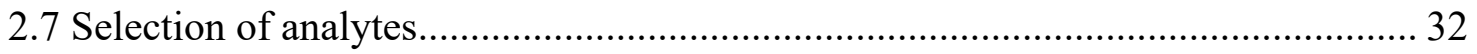

3. PREPARATION OF INCORPORATED/ENTRAPPED, SINGLE ANALYTE

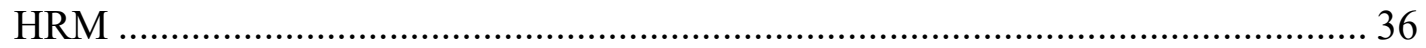

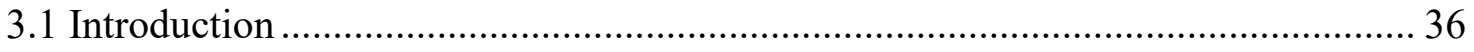

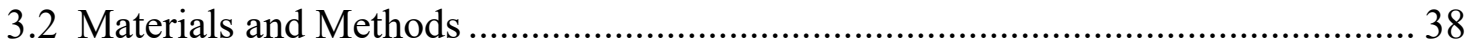

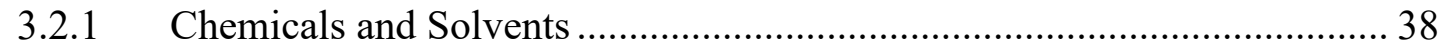

3.2.2 HRM Production by Wet Chemistry-Based Incorporation........................... 39

3.2.3 Isolation of Drugs from Human Head Hair ................................................. 40

3.2.4 Solid Phase Extraction .......................................................................... 41

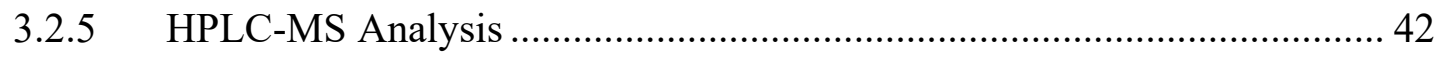




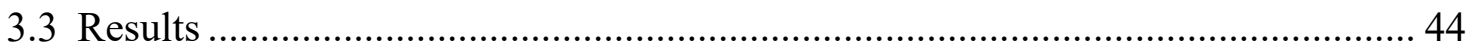

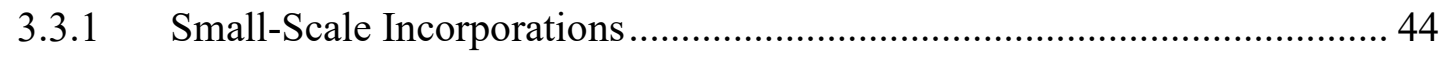

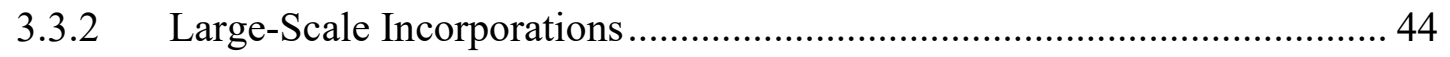

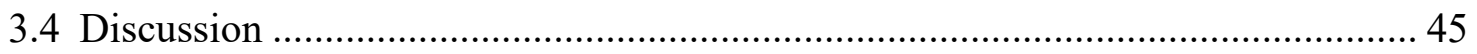

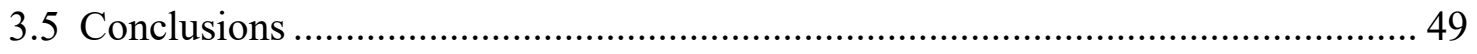

4. SYSTEMATIC COMAPRISON OF HAIR DECONTAMINATION PARAMETERS VIA $2^{4}$ FRACTIONAL FACTORIAL BLOCK DESIGN............ 50

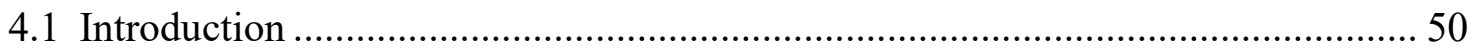

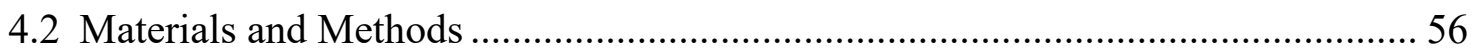

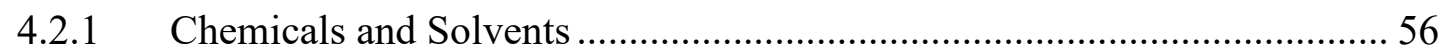

4.2.2 $\quad 2^{4}$ Fractional Factorial Design for Comparing Decontamination

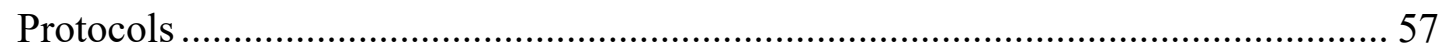

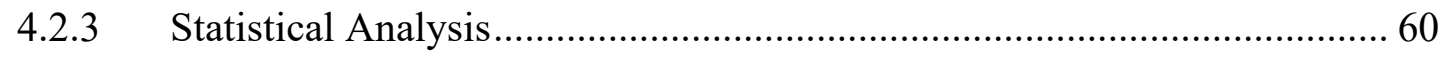

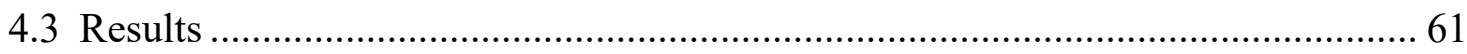

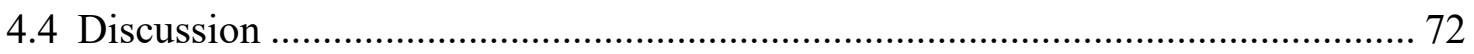

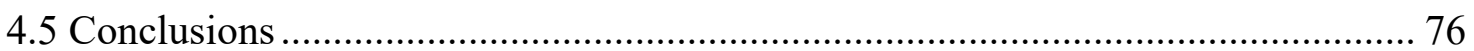

5. SYSTEMATIC COMPARISON OF EXTRACTION PARAMETERS IN FORENSIC HAIR ANALYSIS VIA $2^{3}$ FULL FACTORIAL DESIGN ................. 77

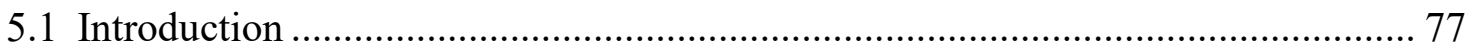

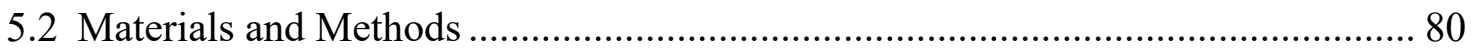

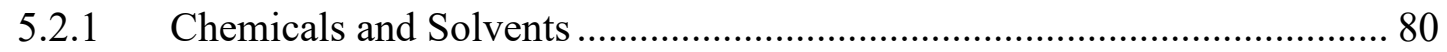

5.2.2 $\quad 2^{3}$ Design Matrix for Comparison of Extraction Technique ....................... 80

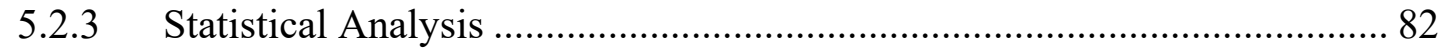

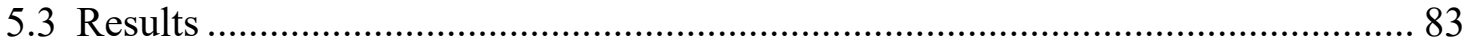

5.3.1 Comparison of Extraction Technique ......................................................... 83

5.3.2 DoE Comparison of Extraction Conditions ..................................................... 84

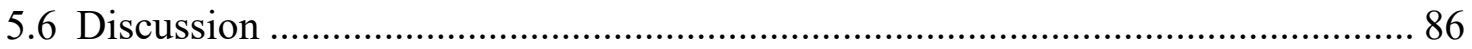

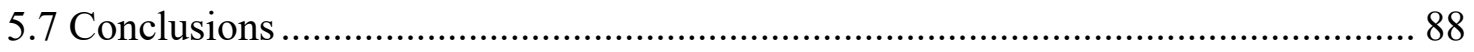

6. PROBING INTERACTIONS BETWEEN DRUGS OF ABUSE AND

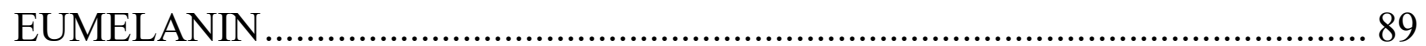

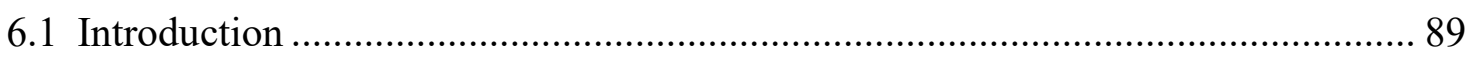

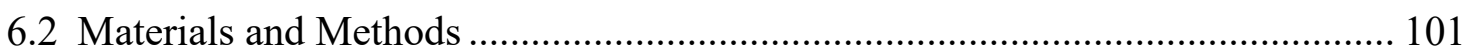

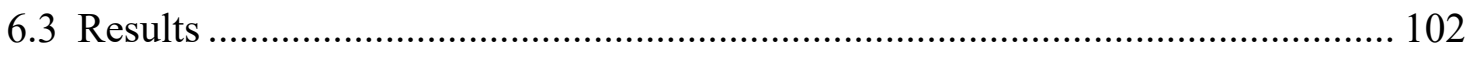




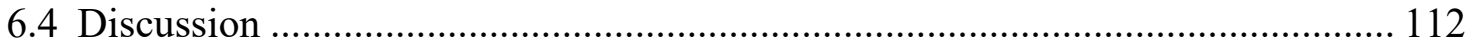

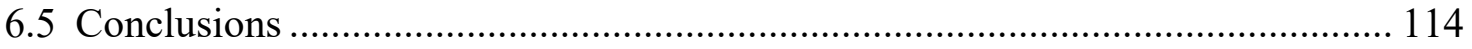

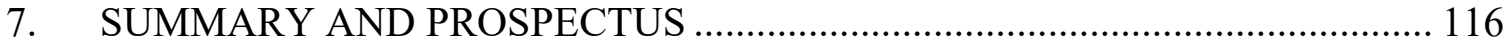

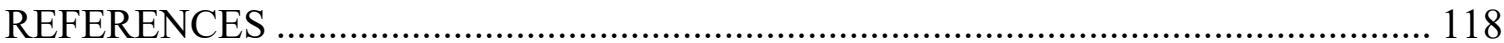

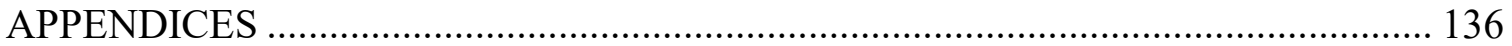

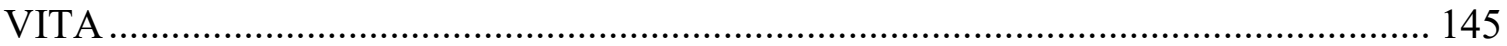




\section{LIST OF TABLES}

TABLE

PAGE

Table 1: Accepted screening and confirmatory cut-off values as reported by the EWDTS. Cut-off values have been approximately agreed upon among regulatory bodies, such as the SoHT, EWDTS, and the substance abuse and mental health services administration (SAMSHA). ${ }^{38,52,53}$

Table 2: Structures and physiochemical properties of the drugs of abuse incorporated into blank hair.

Table 3: MRM transitions selected for LC -MS method.

Table 4: Average recovered drug from small-scale incorporated/entrapped HRM under various buffer conditions.

Table 5: Average recovered drug from large-scale incorporation/entrapment of drugs of abuse in HRM.

Table 6: Augmented $2^{4}$ fractional factorial block design for comparison of decontamination parameters.

Table 7: Definitions of factors and levels of these factors under study in the $2^{4}$

fractional factorial block design. 58

Table 8: MRM transitions used for identification and quantitation of analytes via HPLC-MS.

Table 9: ANOVA for $2^{4}$ fractional factorial block design comparing decontamination parameters for AMP.

Table 10: List of parameters for the design points resulting in the highest recovery of AMP.

Table 11: ANOVA for $2^{4}$ fractional factorial block design comparing decontamination parameters for DZP.

Table 12: List of parameters for the design points resulting in the highest recovery of DZP.

Table 13: ANOVA for $2^{4}$ fractional factorial block design comparing decontamination parameters for HER 
Table 14: List of parameters for the design points resulting in the highest recovery of HER.

Table 15: ANOVA for $2^{4}$ fractional factorial block design comparing decontamination parameters for COC

Table 16: List of parameters for the design points resulting in the highest recovery of COC.

Table 17: ANOVA for $2^{4}$ fractional factorial block design comparing

decontamination parameters for THC 70

Table 18: List of parameters for the design points resulting in the highest recovery of $\Delta^{9}$-THC.

Table 19: Summary of the parameters resulting in the highest average recovery of analytes. 72

Table 20: $2^{3}$ Full Factorial Design for the Comparison of Extraction Parameters 79

Table 21: Definition of factors and factor levels for each experimental design. 82

Table 22: Linearized Langmuir fit parameters and calculated melanin affinities for AMP ephedrine, octopamine, norepinephrine, atropine, and COC. ${ }^{16}$ 95

Table 23: Association constants between eumelanin and drugs according to Borges et al. ${ }^{4}$ 97

Table 24: Recorded $\lambda_{\max }$ values for Drugs of Abuse 103

Table 25: Comparison of association constants for eumelanin and drugs.....

Table 26: Accuracy and Precision Measures

Table 27: Recovery of Analytes Using the Bond Elut Certify Method.

Table 28: Matrix effects observed for each analyte under enzymatic degradation and solvent extraction conditions.

Table 29: MRM Transitions Selected for LC-QqQ Method. 143 


\section{LIST OF FIGURES}

\section{FIGURE}

PAGE

Figure 1: The Morphology of Hair Fibers. ${ }^{18}$ In the upper right portion of the figure, the overall structure of hair is demonstrated, denoting the cuticle, cortex, and the medulla. In the cortex, the presence of a macrofibril within cortical cells, which have a dark outline, is shown. The overall structure of the macrofibril is indicated in the center of the figure, which shows a cluster of intermediate filaments surrounded by intermicrofibrillar matrix. The left portion of the figure shows the structures that make up the intermediate fibril. The $\alpha$-keratins are named as such because their secondary structure is a right-handed $\alpha$-helix. Four $\alpha$-keratins form a two stranded left-handed coiled-coil which, along with other coiled coils and matrix, organize into the left-handed protofibril, which is also known as the intermediate fibril.

Figure 2: Histological longitudinal section of the hair follicle in anagen. ${ }^{2}$

Figure 3: Chemical Structures of Melanin Subtypes (adapted from Ito et al., 2008). ${ }^{21}$ The eumelanin (A) and pheomelanin (B) subtypes of melanin stack on themselves to produce the unique pigments seen in human head hair. The ratios in which these melanin building blocks associate have been suggested to be specific to groups of individuals with the same biogeographical origin. ${ }^{20}$

Figure 4: Scheme of Forensic Hair Analysis (adapted from Vogliardi et al. ${ }^{6}$ ). The process of forensic hair analysis begins with the selection of the segment of hair relevant to the time frame to be investigated. Next, drug superficially associated with the hair surface as a result of external contamination must be removed through a series of decontamination washes. The hair sample is then homogenized and exposed to an extraction solution. The extract is often purified before undergoing qualitative and/or quantitative analysis.

Figure 5: Visual demonstration of ANOVA. ANOVA is capable of determining whether group mean(s) are significantly different from the overall population mean. Subsequent, post-hoc analysis allows for the description of the direction and magnitude of that difference.

Figure 6: General Schematic of Incorporation Technique Employed to Prepare HRM.

A: Drug-free hair is obtained. B: Drug-free hair is soaked in an aqueous solvent, causing the cuticle to swell and partially expose the cortex of the hair shaft.

$\mathrm{C}$ (longitudinal cross-section): After spiking the aqueous solvent with the drug, simple diffusion allows drug to move between the swollen cuticle scales into the cortex of the hair. D: Aqueous solvent is evaporated, allowing the cuticle to smooth back over the cortex. This results in the incorporation of drug into the cortex of the hair and/or entrapment of the drug between cuticle scales. 
Figure 7: Depiction of small-scale and large-scale incorporation schemes.

Figure 8: Analytical scheme for sample preparation for the decontamination DoE studies.

Figure 9: Plot of percent recovery of (A) AMP, (B) DZP, (C) HER, (D) COC, and (E) $\Delta^{9}$-THC detected in the wash solutions for each design point $(\mathrm{n}=3)$. The $y$-axis is the percent recovery of the drug; the $\mathrm{x}$-axis is the design point. Points highlighted in red represent design points with highest recovery. Parameters associated with each design point are summarized in Table 6 .

Figure 10: Schematic of sample preparation for extraction DoE studies.

Figure 11: Comparison of extracted drug with different extraction techniques. Points represent each sample extracted using the designated technique $(n=48)$, with different pre-treatment protocols as outlined in the DoE matrix. The outlines in green represent the quantiles of the data. Solid, central, horizontal line represents the average extracted quantity for each technique for AMP (A), DZP (B), and $\mathrm{THC}(\mathrm{C})$.

Figure 12: Comparing the effect of the variation in extraction parameters on the amount of extracted drug for AMP (A), DZP (B), and THC (C). 86

Figure 13: Monomeric unit of eumelanin. ${ }^{21}$ The carboxylic acid moieties (highlighted in blue) have a $\mathrm{pK}_{\mathrm{a}} \sim 5.0$, and have been hypothesized to interact with cationic drug functionalities under neutral and alkaline conditions (i.e., $\mathrm{pH} \geq 7$ ), in which these carboxylic acids would be deprotonated, and therefore anionic.

Figure 14:Structure of ephedrine. The protons of interest for the study of the dissociation constant with melanin were the N-methyl protons implicit in the blue highlighted area.

Figure 15:Spectra of ephedrine in the absence of melanin (bottom spectrum) and in the presence of melanin (top two spectra). The assignments of some spectral peaks are noted at the top of the figure. The N-methyl peaks, at $2.57 \mathrm{ppm}$, were noted to broaden in the presence of melanin and remain broad after $48 \mathrm{~h}$ and $144 \mathrm{~h} .^{124}$

Figure 16: Fitted trendline for the change in the line width of 2.57 ppm peak of ephedrine titrated into melanin. ${ }^{124}$

Figure 17: Linearized Langmuir isotherms of AMP, ephedrine, octopamine, COC, and norepinephrine. ${ }^{16}$

Figure 18: UV/Vis spectra of AMP with increasing concentrations of melanin. ${ }^{15}$ 96 
Figure 19: MS spectra of AMP with oxidized catechol. A: Single stage scan of mixture showing the characteristic peak of AMP at $135.9 \mathrm{~m} / \mathrm{z}$ and a peak corresponding to the mass of AMP + catechol at $350.1 \mathrm{~m} / \mathrm{z}$. B: Product ion scan with Q1 fixed on the $\mathrm{m} / \mathrm{z}$ of the potential adduct $(350 \mathrm{~m} / \mathrm{z})$. C: Neutral loss scan for a loss of a $214 \mathrm{~m} / \mathrm{z}$ neutral fragment to account for the mass differential between AMP and thepotential adduct. ${ }^{4}$

Figure 20: Energy diagram of electronic transitions that may occur in organic molecules. The two lowest energy transitions (i.e., $\mathrm{n} \rightarrow \pi^{*}$ and $\pi \rightarrow \pi^{*}$ ) are the most probable. These transitions are achievable through irradiation with light ranging from $200-800 \mathrm{~nm}$ (ultraviolet and visible light).

Figure 21: Comparison of spectra for solutions containing $1 \times 10-4 M$ A: AMP, B: COC, C: THC, D: DZP, E: MOR in the absence of melanin (black) and in the presence of $3.0 \mu \mathrm{g} / \mathrm{mL}$ melanin (red). Significant increases in the absorbance at the $\lambda_{\max }$ were observed for AMP, COC, and THC in the presence of melanin, while no significant changes were seen in the spectra of MOR and DZP in the presence of melanin when compared with spectra of these drugs in the absence of melanin.

Figure 22: Linear regressions of the change in $\lambda_{\max }$ values for A: AMP, B: COC, $\mathrm{C}$ : THC with the addition of eumelanin.

Figure 23: Langmuir fit of change in the intensity of absorbance at $208 \mathrm{~nm}$ with the addition of melanin to solutions with AMP at pH 7.4 (red) and 5.5 (blue). 108

Figure 24: Langmuir fit of change in the intensity of absorbance at $208 \mathrm{~nm}$ with the addition of melanin to solutions with AMP at pH 5.5 in $1 \mathrm{X}$ PBS buffer (red) and 10X PBS buffer (blue).

Figure 25: Langmuir fit of change in the intensity of absorbance at the $\lambda_{\max }$ of cocaine with the addition of melanin to solutions with $\mathrm{COC}$ at $\mathrm{pH} 7.4$ (red) and 5.5 (blue)..... 110

Figure 26: Langmuir fit of change in the intensity of absorbance at $214 \mathrm{~nm}$ with the addition of melanin to solutions with $\mathrm{COC}$ at $\mathrm{pH} 7.4$ in $1 \mathrm{X}$ PBS buffer (red) and 10X PBS buffer (blue).

Figure 27: LC separation (from left to right) of morphine, amphetamine, cocaine, heroin, diazepam, and $\Delta 9$-tetrahydrocannabinol at $75 \mathrm{ng} / \mathrm{mL}$. The MRM spectrum of the six separated substances, showing two unique transitions for each and labeled with their respective retention times. 


\section{LIST OF ABBREVIATIONS, ACRONYMS, AND SYMBOLS}

$\begin{array}{ll}\mu \mathrm{g} & \text { micrograms } \\ \mu \mathrm{L} & \text { microliter } \\ \text { AMP } & \text { Amphetamine } \\ \text { ANOVA } & \text { Analysis of variance } \\ \text { COC } & \text { Cocaine } \\ \text { CTS } & \text { Connective tissue sheath } \\ \text { DFC } & \text { Drug-facilitated crime } \\ \text { DHI } & \text { 5,6-dihydroxyindole } \\ \text { DHICA } & \text { 5,6-dihydroxyindole-2-carboxylic acid } \\ \text { DoE } & \text { Design of experiments } \\ \text { DP } & \text { Dermal papilla } \\ \text { DTT } & \text { 1,4-Dithiothreitol } \\ \text { DZP } & \text { Diazepam } \\ \text { EtG } & \text { Ethyl glucuronide } \\ \text { EWDTS } & \text { European Workplace Drug Testing Society } \\ \text { h } & \text { hours } \\ \text { HER } & \text { Heroin } \\ \text { HOMO } & \text { Highest occupied molecular orbital } \\ \text { HPLC-QqQ } & \text { Hair reference material } \\ \text { HRM } & \end{array}$




\begin{tabular}{|c|c|}
\hline HSD & Honestly significant difference \\
\hline IF & Intermediate filament \\
\hline IRS & Inner root sheath \\
\hline $\mathrm{Ka}$ & Association constant \\
\hline $\mathrm{Kd}$ & Dissociation constant \\
\hline $\mathrm{KL}$ & Langmuir constant \\
\hline LC-MS & Liquid chromatography coupled to mass spectrometry \\
\hline LLE & Liquid/liquid extraction \\
\hline LUMO & Lowest unoccupied molecular orbital \\
\hline M & Matrix \\
\hline$m / z$ & Mass-to-charge ratio \\
\hline M-1 & Inverse molar \\
\hline MALDI & Matrix-assisted laser desorption ionization \\
\hline MDA & 3,4-methylenedioxyamphetamine \\
\hline MDMA & 3,4-methylenedioxymethamphetamine \\
\hline $\mathrm{mg}$ & milligram \\
\hline $\mathrm{mL}$ & milliliter \\
\hline MOR & Morphine \\
\hline MRM & Multiple reaction monitoring \\
\hline $\mathrm{n}=$ & number or replicates \\
\hline $\mathrm{N} 2$ & Diatomic nitrogen gas \\
\hline OFAT & One factor at a time \\
\hline ORS & Outer root sheath \\
\hline
\end{tabular}




\begin{tabular}{|c|c|}
\hline PBS & Phosphate buffered saline \\
\hline pg & picograms \\
\hline $\mathrm{ppb}$ & parts per billion \\
\hline ppm & parts per million \\
\hline $\mathrm{S}$ & seconds \\
\hline SAMSHA & Substance abuse and mental health services administration \\
\hline SDS & sodium dodecyl sulfate \\
\hline SoHT & Society of Hair Testing \\
\hline SPE & Solid phase extraction \\
\hline STR & Short tandem repeat \\
\hline $\mathrm{T}$ & Temperature \\
\hline $\mathrm{THC}$ & $\Delta^{9}$-Tetrahydrocannabinol \\
\hline TPSA & Total polar surface area \\
\hline $\mathrm{UV} / \mathrm{Vis}$ & Ultra violet/visible \\
\hline v. & Version \\
\hline$\alpha$ & alpha \\
\hline$\triangle \mathrm{ABS}$ & change in absorbance \\
\hline$\lambda \max$ & wavelength of maximum absorbance \\
\hline$\pi$ & pi \\
\hline
\end{tabular}




\section{INTRODUCTION}

\subsection{Statement of the Problem}

Human head hair has been used as an alternative matrix in forensic toxicology for several decades to overcome some of the challenges and limitations associated with the use of blood and urine for monitoring an individual's exposure to use or abuse of xenobiotic substances. Hair testing is an excellent method for determining drug use history because the window of detection is significantly longer than in blood and urine, allowing for the detection of drugs when traditional testing may fail. While clearly useful for long-term drug detection, hair analysis does have technical and interpretive challenges. As a complex solid sample matrix, hair requires specific pre-treatment measures, including washing, cutting and/or pulverization, and some type of extraction procedure to remove the drug from the hair matrix components to allow for analysis. Optimizing the pre-treatment steps in forensic toxicological analysis of hair and the subsequent extraction of analytes is essential to accurately identify and quantify drugs present in a hair sample. The Society of Hair Testing (SoHT), the only governing body for hair testing to date, has made recommendations on protocols, although they provide only general guidelines for analysis. While numerous methods and a large literature base exists for forensic hair testing, such methods have not been systematically compared with regard to performance. Thus, a major aim of the present work was to develop optimized methods for the pre-treatment and extraction of hair samples to support recommendations toward the standardization of forensic toxicological hair analysis.

Hair is considered an alternative matrix in the field of forensic toxicology since it is not as well studied and understood as more classic matrices like blood and urine, especially in 
regard to the mechanisms by which drugs become associated with the matrix and subsequently held there. Nevertheless, a consensus has emerged that there are three routes of exposure of hair to xenobiotics. ${ }^{1,2,11-13,3-10}$ The first is direct incorporation into hair from blood that contains the drug as it grows from the follicle. Alternatively, substances can become incorporated into hair through contact with drug-containing sweat or sebum on the scalp. Finally, drug-containing aerosols or smoke in an individual's environment can lead to the deposition of drug onto the surface of the hair with subsequent incorporation. While there is a consensus regarding routes of exposure, the exact mechanism by which xenobiotic substances remain on and/or in the hair has not been studied extensively and is poorly understood. The ambiguity of the nature and mechanism of the association between drug and hair components has resulted in criticisms of the routine use of hair as a biological matrix for toxicological analysis. ${ }^{7}$ Therefore, the second major aim of the present work was to study the nature of the forces responsible for the interactions between components of the hair matrix and select drugs of abuse.

\subsection{Rationale for Research}

Contemporary forensic toxicology places high value on validated analytical methods for the qualitative and quantitative determination of drugs of abuse in various matrices. At present, hair is considered an alternative matrix as there are still significant methodological and interpretive challenges that must be addressed. Thus it is important to establish methods for the analysis of drugs of abuse in hair under conditions that maximize the amount of drug that can be isolated from the hair matrix for analysis without also including any amount of drug that is superficially associated with hair structures as a result of external

contamination. Current methods may not be effective in removing all trace of drugs and 
their metabolites from the surface of hair before extracting drug from the matrix nor in extracting the total drug content of the cortex, as the analytes may be associated with the biopolymers present in hair, necessitating a re-evaluation of current techniques. Therefore, more extensive knowledge is required regarding pre-treatment parameters in hair analysis and the mode of interaction between drugs and the components of the hair matrix. Each subtask of the present research was designed to address a specific challenge associated with using hair as a matrix for toxicological analysis.

1.2.1 Task 1 - Preparation of incorporated/entrapped, single analyte hair reference material (HRM).

Several varieties of reference material have been developed for use in hair analysis, the two most common being incorporated/entrapped HRM and authentic HRM. Incorporated/ entrapped HRM is prepared by soaking blank hair in an analyte-containing aqueous buffer. Authentic HRM is derived from individuals who have been exposed to an analyte and whose hair has been decontaminated and tested with a quantitative, validated analytical method. While authentic HRM has the advantage of representing real-world samples, these materials often contain more than one analyte and there is no means to control the concentration along the length of the strands, which may vary as a result of inconsistent use by the individual. Incorporated/entrapped HRM is advantageous because the amount of drug contained in the material is dependent on the known quantity of drug added to the incorporation buffer. In contrast, in authentic HRM the amount of drug in the material is dependent on the variable quantities of drug actively used by the group of individuals sampled for its preparation. In the present work, incorporated/entrapped HRM was used as the amount of drug in the matrix is better controlled, and this this material was prepared to 
contain a single analyte at a time for amphetamine, cocaine, morphine, diazepam, and $\Delta^{9}$ tetrahydrocannabinol.

1.2.2 Task 2 - Systematic comparison of hair decontamination parameters via $2^{4}$ fractional factorial block design.

Hair requires several pre-treatment steps before it can be analyzed for its drug content; these steps include segmentation, decontamination, homogenization, extraction, and purification. ${ }^{6}$ While the approaches to pre-treatment of hair for forensic toxicological analysis are highly variable, the process of decontaminating the surface of hair includes a host of variables in itself. Thus, in the present research, additional attention was given to parameters that often differ between decontamination protocols, including the identities of the organic and aqueous solvent/solutions employed to wash the hair, the number of consecutive washes applied to each sample, the amount of time the hair is exposed to the wash solvent/ solution, and the order of washes applied. Discrete levels of these parameters were applied in a systematic way, using a $2^{4}$ fractional factorial block design. Subsequent ANOVA and Tukey's Honestly Significant Difference (HSD) testing were used to indicate if there were any significant differences in the efficacy of the wash parameters and which wash parameters correlated to those differences, respectively.

1.2.3 Task 3 - Systematic comparison of extraction parameters in forensic hair analysis via a $2^{3}$ full factorial design.

In addition to the decontamination parameters under study, it was important to consider the effect of other pre-treatment steps on the efficacy of the preparation of hair for toxicological analysis. These parameters include the size of the homogenized hair particles, 
the amount of extraction agent the hair samples are exposed to, and the amount of time the hair samples are exposed to the extraction solvent. A $2^{3}$ full factorial design was used to systematically compare discrete levels of these parameters to determine if any factor, or combination of factors, had a significant effect on the extraction efficiency.

1.2.4 Task 4 - Probing physicochemical interactions between drugs of abuse and eumelanin.

Although hypotheses have emerged regarding the means by which drugs become incorporated, entrapped, or associated with the hair matrix, little is known about the mechanisms associated with the retention of drugs in the matrix. The physical and chemical properties of drug substances, specifically lipophilicity, alkalinity, chemical structure, and affinity to major hair components such as melanin, have been suggested to influence their incorporation and retention in hair. ${ }^{3}$ Additional properties of the matrix itself, such as variable rates of growth, porosity, melanin content, chemical treatment, and external contamination, can influence drug incorporation and retention. ${ }^{14}$ Incorporation and retention of substances in hair may, in theory, be the result of non-covalent interaction, such as ionic binding, H-bonding, van der Waals forces, and hydrophobic interactions, and/or to covalent binding with hair components. ${ }^{4,15,16}$ An understanding of these interactions would provide insight into the incorporation mechanisms of drugs into hair, hair color biases that may exist in current testing schemes, and effective methodology for extracting these substances for analysis. Probing of these interactions was conducted using UV/Vis spectroscopy in the present work. 


\subsection{Significance of the Study}

Forensic toxicologists routinely examine blood and urine samples as reliable sources of information about an individual's drug use for testimony given in legal proceedings. However, drugs of abuse have a relatively short window of detection; typically a few hours up to a few days. ${ }^{3}$ Hair can be used to provide a longer window of detection and a temporal profile of drug use for an individual, because it can be segmented to represent different growth periods. While hair testing is useful for historical information regarding drug use, it is necessary to address major challenges in hair testing to improve the consistency of results across laboratories by standardizing hair pre-treatment conditions and to provide additional insight into the mechanisms by which drugs are retained in hair to understand how to reliably recover analytes for routine analysis.

The present research compared the impact of various pre-treatment parameters commonly used in forensic toxicological analysis in human head hair on the recovery of the isolated substances in order to make recommendations for standardized methodology. Additionally, the research investigated the physicochemical mode of interaction between eumelanin and select drugs of abuse by spectroscopic approaches. Consequently, the potential for bias may be minimized as standardized methods are established and the mode

of interaction between hair matrix components and analytes is elucidated. Investigation of the challenges associated with toxicological analysis of human head hair can lead to more accurate techniques that, with time, can prove to be reproducible and therefore be reliable evidence in the courtroom. 


\section{BACKGROUND}

\subsection{Cellular and molecular components of hair fibers}

Hair is a fibrous material with an approximately cylindrical morphology comprised of proteins, lipids, water, melanin, and trace minerals. Melanin accounts for $0.2-1.5 \%$ of hair components, while proteins, mainly keratin, are responsible for $65-95 \%$ of hair composition. ${ }^{17}$ Hair is comprised of two to three subunits: the cuticle and cortex, and, in some cases, the medulla. The outermost unit of hair is the cuticle, consisting of dead and flattened cells that overlay such that they resemble roofing tiles. The edges of these overlaid scale-like cells point toward the terminus of the hair fiber. The cuticle can be up to $1 \mu \mathrm{m}$ thick and serves to protect the cells within the cortex. The cortex contains ortho-, para-, and meso-cortical cells, which differ in cysteine content. ${ }^{18}$ Each of these cells contains $5-$ 8 macrofibrils, which in turn contain $500-800$ microfibrils. These microfibrils consist of type I (acidic) and type II (basic) intermediate filaments (IFs), which are $\alpha$-keratins that combine to form heterodimers (Figure 1). Additionally, the cortex consists of keratin associated proteins, which, along with cytoplasmic and nuclear remnants, serve as the intermicrofibrillar matrix. Coarser and darker hair fibers have a third subunit, the medulla, which is comprised of hollow cells, amorphous proteins, and fine filaments. ${ }^{18}$ 


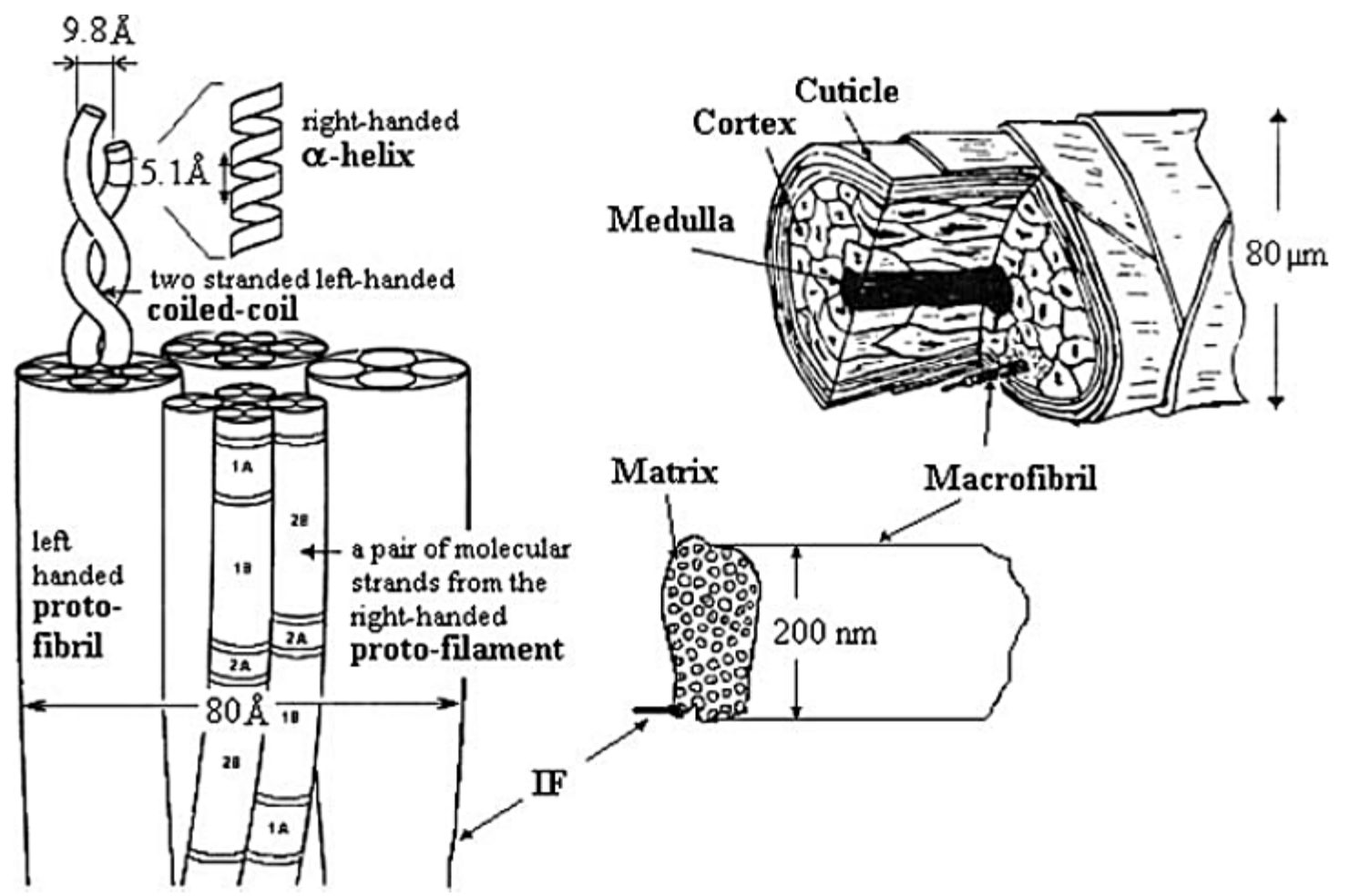

Figure 1: The Morphology of Hair Fibers. ${ }^{18}$ In the upper right portion of the figure, the overall structure of hair is demonstrated, denoting the cuticle, cortex, and the medulla. In the cortex, the presence of a macrofibril within cortical cells, which have a dark outline, is shown. The overall structure of the macrofibril is indicated in the center of the figure, which shows a cluster of intermediate filaments surrounded by intermicrofibrillar matrix. The left portion of the figure shows the structures that make up the intermediate fibril. The $\alpha$ keratins are named as such because their secondary structure is a right-handed $\alpha$-helix. Four $\alpha$-keratins form a two stranded left-handed coiled-coil which, along with other coiled coils and matrix, organize into the left-handed protofibril, which is also known as the intermediate fibril.

Hair fibers form as cells differentiate and organize in the hair follicle, which is an active mini-organ embedded in the epidermis and dermis of the scalp. The hair shaft that is seen outside the scalp is comprised of differentiated, dead keratinocytes that originated from follicular stem cells. Each hair follicle cycles throughout the growth of the hair strand. The cyclic growth of human head hair has three phases, which are anagen, catagen, and telogen. ${ }^{1}$ Anagenesis is the growth phase of hair, when cell division occurs rapidly due to increased metabolic activity. ${ }^{1}$ The intermediate phase of hair growth is the catagen phase, 
where the hair is neither growing nor quiescent, but instead undergoing keratinization. ${ }^{1}$ Blood supply begins to decrease during catagenesis, and is completely halted during telogenesis. The hair follicle is very short during telogenesis, and is easily displaced by arising follicular growth. ${ }^{1}$ At any given time, about $85 \%$ of human head hair will be undergoing anagenesis, $15 \%$ will be in the telogenic phase, and only a small proportion of hair will be in catagenesis. ${ }^{1}$ Specific processes occur in different regions of the hair follicle during each phase of hair growth, leading to the differentiation and subsequent keratinization of stem cells.

There are permanent and non-permanent structures of the hair follicle that are essential for proper hair growth, as can be seen in Figure 2A. The permanent structures are the infundibulum, which is the portion of the follicle that extends from the sebaceous gland to the epidermal surface, and the isthmus, which is the middle part of the hair follicle that is connected to the basal membrane. ${ }^{2}$ The non-permanent structures are present in the hair follicle during anagenesis and include the bulb and the suprabulbular region. The bulb is the thickened end of the hair follicle that contains rapidly proliferating, undifferentiated matrix cells, melanocytes, and outer root sheath cells. ${ }^{2}$ The bulge is comprised of the dermal papilla (DP), matrix (M), the connective tissue sheath (CTS), the inner root sheath (IRS), and the outer root sheath (ORS), as seen in Figure 2C. The tip of the DP is a small, dense cluster of fibroblasts that determines the bulb size, hair shaft diameter and length, and the duration of anagenesis. The DP is surrounded by the matrix, which contains differentiated keratinocytes from the DP and melanocytes, which are rapidly proliferating. The connective tissue sheath is a loose accumulation of collagen and stromal cells that helps keep the bulb in place upon the basement membrane. The outer root sheath contains 
hair follicle stems cells and is directly fed by the matrix. During anagenesis, the isthmus is home to the hair bulge, which is a protrusion of the outer root sheath in the inner-most permanent structure of the follicle, which contains hair follicle stem cells (Figure 2B). The bulge is where differentiated, keratinized cells become organized into the fibrous structures in Figure 1. ${ }^{2}$

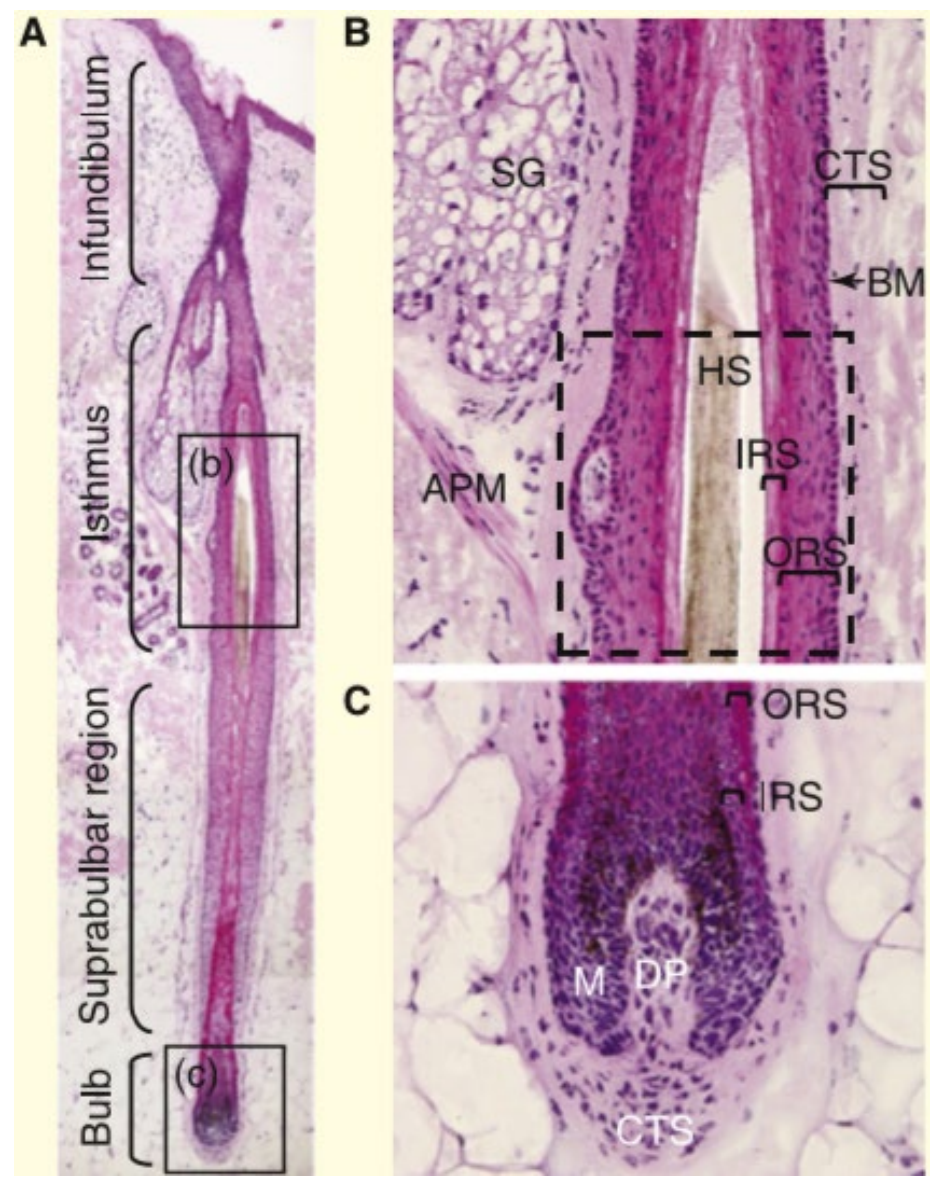

Figure 2: Histological longitudinal section of the hair follicle in anagen. ${ }^{2}$

Keratinization begins at the bulb of the hair root, where alpha helical intercellular structures, called tonofibrils, form via disulfide bond linkage between the keratin proteins of adjacent cells that are attached to intramembrane structures, called desmosomes. ${ }^{19} \mathrm{Next}$, the cells move away from the dermal papillae toward the hair matrix material, containing 
sulfur-rich proteins that allow for rigid structures to form between cells. ${ }^{19}$ Finally, the keratinocytes begin degrading intracellular structures, take on squamous morphology, and move toward the surface of the epidermal layer to grow out of the follicle..$^{2,19}$

As the cells in the follicle differentiate, they are pushed out of the follicle and carry with them granules of the pigment melanin, which is what gives color to human hair and skin. ${ }^{2}$ The pigment molecules are produced in melanocytes during the anagen phase, and become incorporated into the hair structures during keratinization. ${ }^{20}$ Melanin pigment granules are spherical aggregates of oligomeric stacks of melanins, which form after the pigment molecules are synthesized in melanosomes - lysosome-related organelles of the melanocyte which synthesize melanins from tyrosine. ${ }^{2}$

Human hair contains two main subclasses of melanin, eumelanin and pheomelanin, both derived from the biotransformation of tyrosine to dopaquinone and ultimately eumelanin or pheomelanin (Appendix 1). ${ }^{21}$ Eumelanin is a brown/black pigment that contains a high concentration of 5,6-dihydroxyindole-2-carboxylic acid (DHICA). ${ }^{22}$ Pheomelanin, the reddish-brown hair pigment, undergoes a different biosynthetic process resulting in a relatively high concentration of 5,6-dihydrocyindole (DHI). ${ }^{21}$ Additional hair pigments include oxyeumelanin and oxypheomelanin, which are oxidative products of the main melanin subtypes. ${ }^{23}$ Additionally, the different biosynthesis processes involved in producing eumelanin and pheomelanin result in vastly different indolequinone structures, as seen in Figure 3. 
A<smiles></smiles>

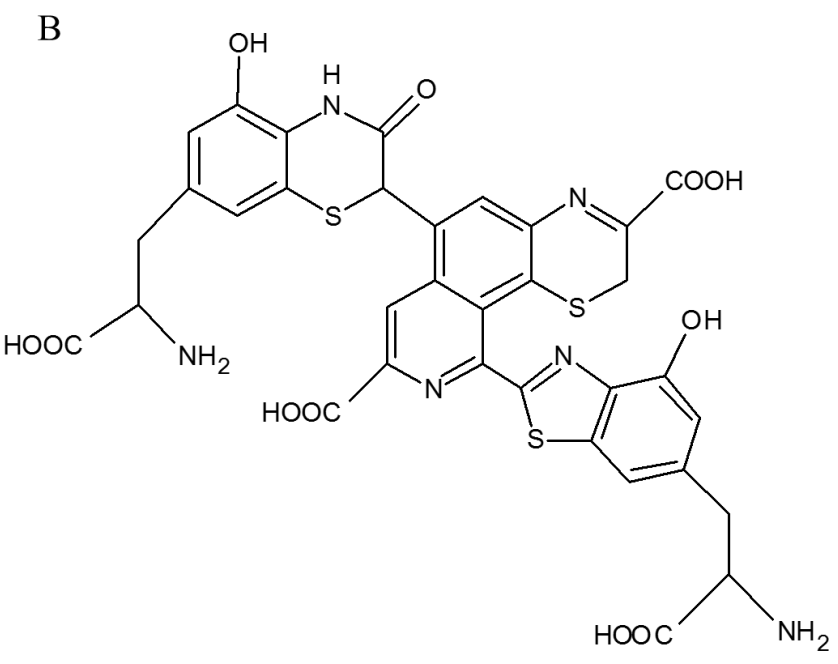

Figure 3: Chemical Structures of Melanin Subtypes (adapted from Ito et al., 2008). ${ }^{21}$ The eumelanin (A) and pheomelanin (B) subtypes of melanin stack on themselves to produce the unique pigments seen in human head hair. The ratios in which these melanin building blocks associate have been suggested to be specific to groups of individuals with the same biogeographical origin. ${ }^{20}$

The most significant structural difference between pheomelanin and eumelanin is the backbone structure. Eumelanin is comprised of indolequinone units, where pheomelanin is comprised of benzothiazine, benzothiazole, and isoquinolone units. Another important structural difference between the two types of melanin found in human hair is the position of the carboxylic acid moieties, as some hypotheses state that these functionalities contribute to melanin affinity for certain molecules. ${ }^{21}$ In the structure of eumelanin, the carboxylic acids are free from steric hindrance and positioned near the secondary amines of the indolequinone backbone. In contrast, many of the carboxylic acid functions in pheomelanin are within two bond lengths of a primary amine, since the amine may donate electron density to stabilize the carboxylic acid in its anionic form, reducing its reactivity. 


\subsection{Incorporation mechanisms of drugs of abuse into hair}

A consensus has emerged that three incorporation mechanisms contribute to drug levels in hair. ${ }^{1,2,3,24,25,4-8,10-12}$ The first involves the systemic circulation of drug-containing blood to the scalp, where simple diffusion causes drug to move from the blood stream into the cells in the hair follicle. The second mechanism involves the secretion of compounds of interest that are dissolved in sweat and sebum onto the scalp, where they can be subsequently deposited on a growing hair shaft. ${ }^{6}$ The third mechanism is deposition of drug onto the surface of hair by exposure to drug-containing aerosols in the surrounding environment; this is a common route of exposure for smoked or insufflated drugs of abuse. ${ }^{6}$

While the routes of exposure that lead to the incorporation of drug into hair have been largely agreed upon, it is still unclear what mechanisms allow for the retention of drugs in the hair shaft. Several theories regarding the physicochemical mode of interaction between drug and the hair matrix have been proposed, ${ }^{4,15,16}$ but none have yet been systematically studied. Retention of drugs and other xenobiotics in hair may be a result of interactions between analytes and the components of hair, such as melanin and keratin, through covalent or ionic binding, van der Waals forces, hydrophobic interactions, or a combination thereof. The interactions between drugs and hair are likely to be specific to the physical and chemical properties of the analyte. It has been suggested that basic drugs of abuse may have covalent interactions with melanin, while acidic drugs may have interactions with other hair proteins, whether covalent or otherwise. ${ }^{6,13}$ It is theorized that analytes that are cationic at physiological $\mathrm{pH}$ interact with the anionic carboxylic acids of the melanin polymer; these electrostatic interactions are further strengthened by van der Waals forces between aromatic rings in the drug molecules and the aromatic indole rings of melanin. ${ }^{23}$ 
While it has been demonstrated that there are different binding modes of analytes with different physical and chemical properties, the nature of these associations are not well understood.

\subsection{Hair as forensic evidence}

Human head hair has historically been studied as evidence in several contexts. For example, hair has been examined under the lens of fiber analysis to study the structures of the cuticle, medulla, and cortex to determine its biological origin, as these structures differ among mammalian species. ${ }^{26}$ Additionally, the microscopic evaluation of hair structures allows for the classification of a hair fiber according to its corporeal location or origin. Head hair is documented to have an intermediate thickness, $60-80 \mu \mathrm{m}$, where pubic and axillary hair tend to be thicker and coarser than hair on the scalp, and vellus hair is thinner and less coarse than both scalp and pubic/ axillary hair. ${ }^{27}$ Additional measures that can be used to differentiate the corporeal origin of a hair fiber including the presence/ absence and thickness of the medulla as it has been observed that pubic and axillary hair is more medullated than scalp hair and vellus hair. ${ }^{28}$ Also, the medullary index, or the ratio of the thicknesses of the medulla as compared to the thickness of the whole strand, has been shown to be greater in pubic and axillary hair than in scalp hair. ${ }^{28}$

In addition to species and corporeal provenance, hair specimens can undergo DNA extraction and subsequent genotyping to trace the sample to a subpopulation or even an individual. When hair strands are pulled from their source, the follicle may be removed with the hair strand; since the follicular cells are not yet keratinized, nuclear DNA is still intact and can be extracted for genotype analysis to attribute the profile to a single individual. ${ }^{29}$ At present, the lack of follicular material dictates that a sample will undergo 
mitochondrial DNA analysis, which can provide matrilineal information about the source, but cannot be used for short tandem repeat (STR) analysis, which the is benchmark technique for forensic DNA profiling to identify an individual. ${ }^{29}$ Recent developments have been reported to indicate that modification of the DNA extraction protocol and STR primer sets may allow for the identification of a single individual from rootless hair. ${ }^{29}$ While the provenance of hair is an important use of hair evidence, the focus of many recent developments in the last two hundred years regard the toxicological information that can be ascertained from this matrix.

\subsection{Hair as a matrix in forensic toxicology}

Forensic toxicological analysis for the purposes of protecting human health and supporting criminal justice activities can be achieved, in part, by means of analysis of human head hair for detection of abused substances. Forensic drug analysis in any biological matrix serves to monitor the exposure to a variety of substances, recognize prenatal drug exposure, evaluate abstinence from drug use, and detect exposure to drug facilitated crime substances. Blood and urine are the most commonly tested biological matrices for detecting the use or exposure to drugs, and other compounds of interest, because these matrices are well understood and standard operating procedures exist. ${ }^{1}$ Alternatives to blood and urine include hair, nail clippings, and oral fluid as biological matrices. ${ }^{1}$ These have been explored because their collection involves a less invasive process than collecting blood or urine from an individual. Additionally, some alternative matrices, like hair and nail clippings, provide information that blood and urine cannot. The longer window of detection in hair and nails is a result of growth on the body over time, 
which allows for the detection of substances at different times within the lifetime of the hair strand or nail via segmental analysis. ${ }^{3}$

The first report of toxicological data from human hair was by Hoppe in a German publication describing results of a study of suspected arsenic poisoning. ${ }^{30}$ The next notable publication regarding the detection of xenobiotics in hair occurred almost a century later; Goldblum et al. dosed guinea pigs with barbiturates and subsequently detected these drugs in the hair of guinea pigs. Drug testing in hair became of particular interest beginning in the 1980s. The surge began with Baumgartner et al. extracting opiates from hair. ${ }^{31} \mathrm{~A}$ year later, in Germany, Klug published a method to completely degrade the hair matrix with sodium hydroxide and analyze the codeine and morphine content by immunoassay and confirmed these results with chromatography, which made this method the first to fulfill the requirements of methods used in forensic toxicology.$^{30}$ Next, Valente et al. studied cocaine and its metabolites under different extraction conditions to determine which method resulted in the lowest detection limits. ${ }^{32}$ The methods established to this point had used alkaline or acidic degradation of the hair matrix to isolate drugs for subsequent analysis. In 1985, Haley and Hoffman introduced a new method that used acetone and ultrasonication to extract nicotine and cotinine from hair. ${ }^{33}$ Subsequently, protocols to degrade hair via proteolytic enzymes were established using pronase and proteinase $\mathrm{K}$ by Offidani et al. and Harkey et al., respectively. ${ }^{34,35}$ In the early 1990s, the isolation of drugs from hair by means of supercritical fluid extraction were reported. ${ }^{36,37}$

Over time, many of these influential contributors to early hair testing methods recognized the necessity of establishing quality assurance measures, which led to the establishment of the only governing body for forensic hair analysis: The SoHT. The SoHT 
has made recommendations on a general scheme of analysis. ${ }^{38}$ These recommendations provide an understanding of the steps that are minimally necessary for hair analysis, while suggesting that laboratories investigate and validate methods to fit within these guidelines.

Prior to forensic toxicological analysis, human head hair requires significant processing. Pre-treatment steps in the analysis of drugs in hair include decontamination and segmentation of the hair strand, extraction of the compounds of interest from the hair matrix, and often, purification of the isolate. ${ }^{6}$ Different mechanisms to recover drugs from hair are used with different extraction techniques, e.g., alkaline or enzymatic degradation of the hair proteins or solvent swelling of the hair structures.

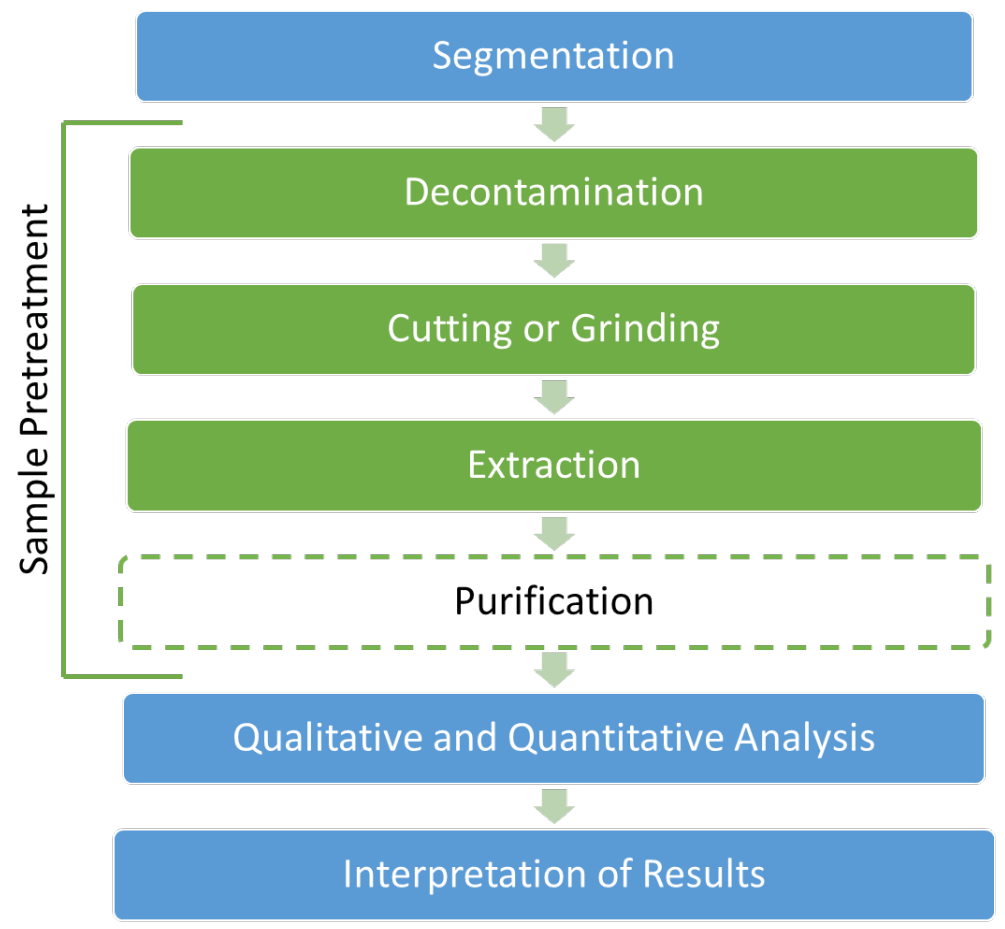

Figure 4: Scheme of Forensic Hair Analysis (adapted from Vogliardi et al. ${ }^{6}$ ). The process of forensic hair analysis begins with the selection of the segment of hair relevant to the time frame to be investigated. Next, drug superficially associated with the hair surface as a result of external contamination must be removed through a series of decontamination washes. The hair sample is then homogenized and exposed to an extraction solution. The extract is often purified before undergoing qualitative and/or quantitative analysis. 
Over time, a great variety of methods for hair analysis have been developed and validated, resulting in a practical challenge, as there is little reported work rigorously comparing the benefits and limitations of using one method over another. ${ }^{6,39-44}$ The process begins with the collection of the sample. Hair growth rate has the lowest intraindividual variation at the posterior vertex of the head, and thus is the ideal location for sample collection. ${ }^{38}$ While the SoHT does not make recommendations regarding adequate sample mass, literature reviews suggest that 10 to $200 \mathrm{mg}$ of hair should be sampled for subsequent analysis. ${ }^{6,39,45}$ It is important that the sample orientation be retained such that the proper segment of hair may be selected for analysis and correlated to an accurate time of exposure.

Once the segment of interest has been cut from the strand, it must undergo a decontamination process to remove any drug that may be superficially associated with the surface of the hair as a result of environmental contamination. $3,6,12,38,45-48$

The goal of the decontamination process is to remove contaminants from the surface of the hair fiber without extracting any of the analyte(s) contained within. The SoHT recommendations dictate that the hair be exposed to an aqueous solvent to remove polar contaminants and an organic solvent to eliminate nonpolar contaminants. ${ }^{38}$ It is common for surfactants, most commonly sodium dodecyl sulfate (SDS), to be added to water for the aqueous decontamination step. ${ }^{6,45,46}$ Common organic solvents used for the purpose of decontamination include protic solvents such as methanol and ethanol and aprotic solvents such as methylene chloride and acetone. ${ }^{39}$ Once the sample has been decontaminated, it must be dried and homogenized. Although the SoHT reports that cutting the hair into smaller segments or pulverizing the hair are both acceptable methods for the homogenization of the sample, it has been reported that pulverization results in 
significantly higher recovery of the drug contained within the hair, as smaller particles increase the surface area of the cortex exposed to the extraction agent. ${ }^{45,46,49,50}$ Extraction of analytes from within the hair has been achieved through several means, including techniques designed to degrade the keratinized sample matrix and those designed to swell the hair matrix, allowing for passive diffusion of the analytes out of the hair and into the extraction solution.

The SoHT recommends comparing different extraction techniques when developing a new protocol to determine the conditions under which analyte degradation is minimized and recovery is maximized. Some techniques, such as enzymatic, alkaline, and acidic conditions degrade the keratinized structures of the hair matrix. ${ }^{6,38,39,43,45,46}$ Alternatively, application of individual solvents, such as methanol and buffers, or mixtures of solvents has been used as a gentle and robust method for isolating drugs from the hair matrix by allowing the imbricate cuticle cells to flare away from the cortex. ${ }^{6,38,39,46,51}$ Although alkaline and acidic degradative techniques have been noted to have high extraction efficiencies for many drugs of abuse, these methods have also been known to cause hydrolysis of certain analytes, such as cocaine..$^{6,38,39,45}$ Enzymatic degradation of the keratin matrix has been reported to have high extraction efficiency under appropriate $\mathrm{pH}$ and temperature conditions to minimize the chemical modification of drugs and their metabolites. However the presence of enzyme, usually proteinase K, proteinase E, or pronase, may result in denaturation of antibodies in immunoassays, which are a common analytical technique for toxicological screening. ${ }^{6,46}$ Solvent-swelling techniques are useful for unknown screening to minimize the risk of chemically modifying analytes, but these typically result in lower extraction efficiency as compared to degradative techniques. ${ }^{6,38,46}$ 
In addition to the SoHT, the European Workplace Drug Testing Society (EWDTS) published guidelines for each step of hair testing. The EWDTS and SoHT have similar guidelines, ultimately stating that each testing lab must validate its own method. Like the SoHT, the EWDTS dictates that sample collection occur from posterior vertex of the head and the amount of hair collected should be about the thickness of a pencil $(0.5-0.5 \mathrm{~cm})$ in diameter with root end clearly marked. Decontamination is a necessary step and can be any combination of aqueous and organic solvents that is validated by the lab. A validated extraction protocol must be established and may involve drying, pulverizing, cutting into small pieces, or chemical disintegration of sample. Screening by immunoassay is recommended to eliminate negative samples; subsequently a validated GC- or LC-MS method may be used for confirmation of presumptive positives. To improve the signal-tonoise ratio in the MS based methods, clean-up of samples prior to analysis is recommended, which may include LLE or SPE. The EWDTS has specific guidelines for internal quality control including low and high control samples at the start and end of a batch analysis. The low level should be around the accepted cut-off value to indicate a positive result (Table 1). Additionally, it is recommended that each lab participate in annual proficiency tests as a form of external quality control. ${ }^{52}$ 
Table 1: Accepted screening and confirmatory cut-off values as reported by the EWDTS. Cut-off values have been approximately agreed upon among regulatory bodies, such as the SoHT, EWDTS, and the substance abuse and mental health services administration (SAMSHA). ${ }^{38,52,53}$

\begin{tabular}{lcc} 
& Screening $n g / m g$ & Confirmation $n g / m g$ \\
\hline Alcohol (segment 0-3) & - & EtG: 0.03 \\
& & FAEE: 0.5 \\
Amphetamines & 0.2 & \\
Amphetamine & & 0.2 \\
Methamphetamine & & 0.2 \\
MDMA & & 0.2 \\
MDEA & & 0.2 \\
MDA & 0.5 & 0.2 \\
Cocaine & - & 0.5 \\
Benzoylecgonine & - & 0.05 \\
Cocaethylene & - & 0.05 \\
Norcocaine & & 0.05 \\
Cannabinoids & 0.1 & \\
THC & - & 0.05 \\
THC-COOH & 0.2 & 0.0002 \\
Opiates & & \\
Morphine & & 0.2 \\
Codeine & & 0.2 \\
6-MAM & & 0.2 \\
Methadone & & 0.2 \\
EDDP & & 0.05 \\
Buprenorphine & 0.2 & 0.01 \\
Norbuprenorphine & & 0.01 \\
Ketamine & 0.01 & 0.5 \\
Norketamine & & 0.1 \\
Benzodiazepines/z-drugs & &
\end{tabular}

Once the analytes have been freed from the matrix, it is necessary to eliminate interferences and isolate analytes. Common clean-up methods for hair extracts include liquid/liquid extraction (LLE) and solid phase extraction (SPE). Clean-up via LLE exploits the differential solubility of an analyte in various solvents and is a widely accepted, reproducible sample preparation technique. Limitations to LLE include emulsion formation and the necessity of large solvent volumes. ${ }^{46}$ Alternatively, SPE uses less solvent 
than LLE and serves the combined purpose of separating analytes from matrix components while simultaneously preconcentrating the sample. However, the high surface area of typical SPE sorbents can result in low recovery of analytes present in trace amounts and a strong affinity for the sorbent. ${ }^{46}$ Once interferences and matrix components have been removed from the extracts, a number of qualitative and/or quantitative analytical techniques may be applied to identify and/or quantify the analytes present in the sample.

Common analytical techniques include immunoassay, GC-MS, and LC-MS. The most recent developments in hair testing have been applied to studying drug use history over the course of time through mass spectrometric imaging of hair strands from a single individual. These techniques predominantly employ matrix-assisted laser desorption ionization (MALDI)-MS and was first reported in 2011 for the analysis of cocaine and its metabolites. ${ }^{54-56}$ Analysis of the longitudinal cross-section of a single hair allows for the imaging of drug species over the length of the hair strand, which then can be correlated to a specific time in an individual's drug use history. Other similar methods have been developed for the detection of methamphetamine, cannabinoids, ketamine, zolpidem, and tilidine..$^{54,56,65,66,57-64}$ The selection of the appropriate analytical technique, or combination of techniques, should be chosen to fit the purpose of the analysis.

\subsection{Challenges in hair testing}

It is evident that each step in sample preparation and analysis can vary significantly among laboratories, resulting in a wide variety of approaches to toxicological hair analysis. In fact, as advances are made in separation sciences and spectroscopy, the analytical approaches to hair analysis may continue to evolve as well. In terms of the forensic application of toxicological hair analysis, it is important to recognize that the results of hair 
testing are often presented in court and have real implications in the lives of individuals. Therefore, it is necessary to establish specific standard practices for the toxicological analysis of hair such that it is clear what evidence is acceptable for use in the court of law.

In addition to the lack of standardization, other major challenges in forensic hair analysis are recognized, including a lack of reliable HRM and complex interpretation of results. A large literature base exists for a variety of analytical techniques and pretreatment steps in the analysis of head hair for its content of drugs of abuse. ${ }^{1,3,6,7,39,66-68}$ However, to date, there are no widely accepted, optimized methods for specific drugs or classes of drugs. More importantly, there has not been a rigorous statistical comparison of hair processing techniques with regard to performance., ${ }^{7,49}$

Additionally, the validated methodologies reported in the literature do not have a "ground truth" reference material with which the method can be tested for accuracy, precision, and to fully understand the matrix effects. Currently available HRM are generally prepared by external "incorporation" of the drug by soaking in a spiked aqueous solution, which is substantially different than the mechanism by which drugs are incorporated into the matrix in vivo. Thus, HRM are often inhomogeneous and poorly representative of real samples. Additionally, with current techniques for the preparation of reference material, it is difficult to incorporate metabolites of drugs because they are more polar than parent compounds and less likely to undergo passive diffusion from the incorporation buffer into the hair matrix. The difficulty incorporating metabolites into hair is a problem because metabolites are valuable as biomarkers of exposure to a substance. Unfortunately, at the present time, most xenobiotic substances relevant to forensic case work are not available as incorporated drug standards in human head hair. 
Finally, the interpretation of hair testing results often requires other information in addition to the analytical data. The concentration in hair is related to the dose of consumed drug, but this relationship is not as well understood as it is for blood and urine. ${ }^{14}$ Interindividual difference in drug metabolism contribute to the complexity in interpreting results. Also, the distance from the hair root that the sample was acquired is a complicating factor, as drug concentrations are typically lower farther away from the root, as a result of UV light exposure and regular hygiene practices like washing. In addition, the polarity of the drug and the hair color contribute to interindividual differences in detected drug concentrations. The proportions of hair in the anagen and telogen phases of growth will contain different concentrations of drug, as incorporation related to consumption only occurs while the hair follicle is in a growth phase and exposed to drug through systemic circulation. Finally, cosmetic treatments must be considered during interpretation of hair testing results, because cuticle damage can be caused by perming, straightening, dyeing excessive washing, bleaching, and UV exposure. These treatments also can increase hair porosity, allowing for drug to escape the matrix and increasing the potential for false negatives. $^{52}$

One of the greatest challenges and sources of uncertainty in the toxicological analysis of hair involves decontamination prior to extraction and analysis. Numerous reports exist evaluating decontamination procedures for removing external drug contamination and subsequent data interpretation to ensure that measured hair levels only reflect ingested drug transported via systemic circulation to the hair follicle capillaries. ${ }^{66,67,69-76}$ To achieve decontamination, drug superficially associated with the cuticle of the hair must be removed. External contamination may arise from environmental exposure to smoked or 
powdered drug or via surface contact with drug excreted in sweat or sebum. Important considerations for hair decontamination include choice of wash solution or solvent, the quantity of wash solution or solvent used relative to the amount of hair to be decontaminated, the number of consecutive washes to be performed, and the length of time hair is agitated in the decontamination wash solutions or solvents. ${ }^{69,70}$ The SoHT has endorsed the use of an aqueous wash and an organic wash. ${ }^{38}$ However, these recommendations are general, and thus a large number of specific wash procedures have emerged with a variety of efficacies.

To account for interpretive issues that arise from the external contamination, the EWDTS has additional confirmatory regulations. To confirm the consumption of $\mathrm{COC}$, at least two of the following must also be detected: benzoylecgonine, cocaethylene, and hydroxycocaines. Heroin consumption is confirmed by the presence of 6-acetylmorphine and morphine in addition to HER. The consumption of methamphetamine must be confirmed by the presence of AMP in addition to the parent drug. Cannabis consumption is confirmed by the presence of $\mathrm{THC}$ and $\mathrm{THC}-\mathrm{COOH}$. The presence of 3,4methylenedioxymethamphetamine (MDMA) and its metabolite 3,4methylenedioxyamphetamine (MDA) are necessary for confirmation of consumption. Likewise, the confirmation of ketamine requires the presence of its metabolite norketamine. Finally, the consumption of methadone consumption requires the additional presence of its metabolite EDDP. ${ }^{52}$ The FBI has recently adopted similar methods that require the identification of metabolites in addition to parent drug to qualify a positive result for cocaine. ${ }^{77}$ However, interindividual differences in drug metabolism, hair 
structures/texture, and the poor rates of incorporation into hair make quantitation of drugs and their metabolites unreliable. ${ }^{78}$

A large literature base serves to provide an analyst with a breadth of techniques that have been validated for the qualitative and quantitative detection of numerous drugs of abuse in hair while accounting for some of these challenges. Two approaches to method development are common threads amongst the many reports; the development of techniques designed to extract and identify/quantify as many analytes as possible and the development of a specific technique designed to extract and quantify a particular compound or class of compounds. The variation in analytical approaches is greatest in regard to the pre-treatment steps taken to prepare the specimen for quantitative/qualitative analyses. The present work aims to address the challenges of uncertainty regarding the mechanism by which drug is held in hair by probing these interactions via spectroscopy; addressing this challenge helps to account for interindividual differences in hair structure/color when interpreting results. The present work will also address the variability in pre-analytical methodology, with a special focus on decontamination, to account for external contamination and interlaboratory differences.

\subsection{Introduction to Statistical Design of Experiments}

Statistical Design of Experiment (DoE) is an alternative approach to analyzing the associations between independent and dependent variables that is commonly used for product design and process improvement in a multitude of scientific fields. Traditionally, the approach to determining the effect of each independent variable on the dependent variable is to vary the levels "One Factor at a Time" (OFAT) to assess their individual effects on the response, or dependent variable. Subsequently, another variable can be tested 
at different levels while holding the first variable and the others at constant values. The OFAT method of testing requires an abundance of time and materials, making it inefficient and often infeasible. Alternatively, DoE allows for the variation of all parameters simultaneously in order to observe the associations between changes in the dependent variable and the changes in each of the factors and combinations of factors (i.e., “interaction effects"). ${ }^{79,80}$

The theory behind DoE is derived from the statistical approach to hypothesis testing. The goal is to determine if a statement about a population parameter is true based on a random sample. In general, hypothesis testing, and thus DoE, includes testing a null hypothesis against an alternative hypothesis, with the intent of minimizing the probability of rejecting the null hypothesis when it is true (type I error). Minimizing type I error reduces the probability of false positives. Therefore, a significance level must be chosen such that the probability of type I error is minimized. Once this is done, an experiment can be conducted on a representative population of samples, and statistical analysis (such as a Student's t-test) can be conducted to compare the experimental parameter to the total population parameter. If there is no significant difference between these parameters at the chosen significance level then there is insufficient evidence to reject the null hypothesis.

When comparing more than one parameter, a more sophisticated statistical analysis must be used, such as analysis of variance (ANOVA). The ANOVA model, in its simplest form, can be seen in Equation 1, where $Y_{i j}$ is the continuous, numeric response of the dependent variable for the $\mathrm{j} t h$ observation of treatment $\mathrm{i} t h, \mu$ is the overall population mean of the response, $\tau_{\mathrm{i}}$ is the deviation of the group mean from the overall mean, and $\varepsilon_{\mathrm{ij}}$ is the deviation caused by random error. ${ }^{79}$ 


$$
Y_{i j}=\mu+\tau_{i}+\epsilon_{i j}
$$

Equation 1

The purpose of ANOVA is to identify if there is a source of variance in a data set associated with different treatments applied to groups of samples $(\tau)$, rather than random error $(\varepsilon)$. As seen in Figure 5, by plotting the variation of samples within treatment groups versus the overall population mean, the magnitude and direction of the variance resulting from treatment can be determined. ${ }^{79}$ Simply stated, ANOVA separates the variance in a data set associated with a treatment from the variance associated with random error. When the random error is "purified" in this way, the power of the analysis is increased because the F statistic becomes more accurate when sources of known error are teased out of the random error.

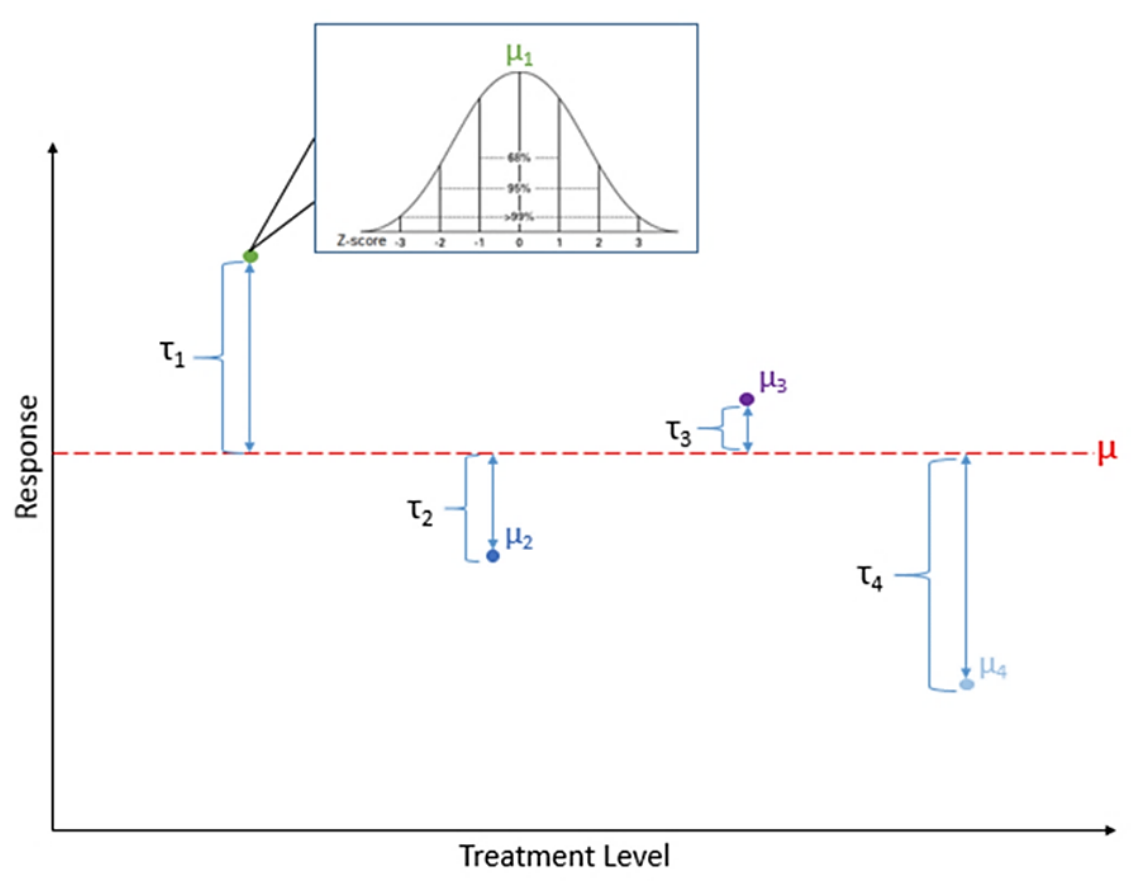

Figure 5: Visual demonstration of $A N O V A$. ANOVA is capable of determining whether group mean(s) are significantly different from the overall population mean. Subsequent, post-hoc analysis allows for the description of the direction and magnitude of that difference. 
An ANOVA F-test is subsequently used to determine if the mean of the treatment groups are significantly different than the population mean (i.e., the variance between the groups is larger than the variance within groups).$^{79}$ If the variation resulting from treatment is significant at a chosen significance level, then there is insufficient evidence to reject the null hypothesis that the treatment does not affect the outcome, on average.

The ANOVA F-test is only capable of determining if the treatment group mean is significantly different than the overall population mean; it is not capable of telling which groups are significantly different, or by how much. Instead, post-hoc analysis is applied for this purpose. When comparing the effect of several treatments, type I error is inflated by multiple-comparisons (i.e., multiple t-tests). Post-hoc testing adjusts the significance level to account for a family-wise type I error, thus minimizing the potential for overall type I error. Tukey's HSD is the best method for pair-wise comparisons while adjusting the overall error rate. ${ }^{79}$

There are a variety of DoE approaches, each with its own purpose. Factorial designs, which are used to consider two or more treatment factors, especially when the interactions among these factors are of interest, are especially useful in the beginning stages of experimental work involving many factors of interest. The purpose of a factorial design is to screen all possible combinations of the levels of the factors of interest, which are studied in each complete trial of the experiment while minimizing the number of runs. Factorial design types are defined by the number of levels of each factor under study and the number of main factors of interest, i.e., independent variables. Nuisance factors are any variables that are difficult to control and result in an inability to perform all the runs of an experiment under homogeneous conditions. In some cases, there are two or more nuisance factors; the 
result is such that two or more "blocking factors" must be used. Randomization of the run order is necessary to eliminate systematic error. Sometimes, it is not practical or feasible to completely randomize all the runs necessary to complete a trial of the experiment as a result of nuisance factors, necessitating the use of blocks within the design. In this case, it is necessary to confound the design; confounding a design involves arranging the complete design into blocks that are smaller than the total number of treatment groups. The consequence of confounding a design is information loss, usually from higher-order effects as these effects become indistinguishable from the those of the blocking factors. ${ }^{79}$ These types of designs are referred to as fractional factorial designs. Specific examples of factorial and fractional factorial designs are given in Chapters 4 and 5 as they relate to the research herein.

Applications of DoE include optimization of cell culture conditions and processes to increase the yield of useful cell products like biopharmaceuticals, optimization of synthetic conditions to result in high yield and desirable products, and the optimization of HPLC methods for the separation of analytes ${ }^{81-86}$ For example, Debrus et al. used a full factorial design to optimize an HPLC method for the analysis of pharmaceuticals considering the mobile phase $\mathrm{pH}$ and gradient times as the main factors. The HPLC method was developed using a $\mathrm{C}_{18}$ reverse-phase column, which has a $\mathrm{pH}$ tolerance from 2.6 to 10 ; five buffers were prepared with different $\mathrm{pH}$ values within this range for the DoE runs. Linear gradients from $95 \%$ aqueous to $5 \%$ aqueous were employed; three levels, or gradient times, ranging from 10 min to 30 min were used in the DoE runs. The dependent variable, or response factor, was the resolution of the peaks in the resultant spectra. The result of these experiments determined that the optimal resolution between the chromatographic peaks 
was obtained using a buffer at $\mathrm{pH} 3.1$ with a gradient time of $30 \mathrm{~min} \cdot{ }^{84}$ Similarly, De Backer et al. used a full factorial design to optimize HPLC chromatographic conditions with the aim of optimizing the resolution of the chromatographic peaks resulting from the reversephase separation of a variety of natural cannabinoids. The factors under study were the percent of organic mobile phase at the start of the gradient, the gradient time, and the $\mathrm{pH}$ of the aqueous mobile phase. The results indicated that the optimal resolution between the chromatographic peaks of the cannabinoids occurred when the gradient started with $68 \%$ organic mobile phase, followed by a 30 min linear gradient with an aqueous buffer at $\mathrm{pH}$ 5.2 .85

There are two types of multivariate approaches to analytical method optimization; these are screening designs and optimization designs. Screening designs are typically used as a first step in the DoE process to determine which, if any, of the parameters of interest have significant impact on the response. Subsequently, these parameters may be optimized. The designs commonly used for screening purposes are the Plackett-Burmann design and $2^{\mathrm{k}}$ factorial designs. ${ }^{81,84}$ Plackett-Burmann designs are limited in that they are not able to determine the significance of high-level interaction factors, where $2^{\mathrm{k}-}$ factorial designs can. ${ }^{50}$ Once the significant factors have been identified, they can be optimized through a full factorial, central composite, or D-optimal design. ${ }^{84}$ The full factorial design includes all combinations of all factors as design points. ${ }^{79}$ The central composite design places experiments around the design center in the design space, which indicate the optimum conditions. ${ }^{81}$ D-optimal designs are useful when the design space is constrained and the covariance of parameters is low..$^{84,87}$ 


\subsection{Selection of analytes}

Analytes of interest, specifically AMP, COC, MOR, and THC, were identified from commonly abused substances and from substances for which cut-off values have been established by the SoHT. ${ }^{38}$ Diazepam was chosen as an analyte because it is a widely used prescription drug that is also used in drug facilitated crimes (DFC), which is a common use of toxicological data derived from hair samples. ${ }^{43}$ These analytes have shown moderate to high abuse potential, which is defined in by studies in which individuals are given controlled doses of the drug and report the positive and negative effects they experience and their likelihood of using again based on those experiences. ${ }^{88-92}$ Forensic hair analysis may be performed looking for DFC cases, as was previously noted specifically for DZP, child custody cases, driver's license reissuing, and workplace drug testing. ${ }^{47,52,57,93}$

The physical and chemical properties of substances, including lipophilicity, acid-base properties, chemical structure, and affinity to melanin, have been cited to influence their incorporation and retention in hair. ${ }^{1,3,7,25,47}$ Therefore, the properties of select drugs of abuse were considered when choosing the analytes for this work. Lipophilicity can be understood through the $\log \mathrm{P}$ value of the substance, which is a partition coefficient that allows for the understanding of the neutral molecule's affinity for octanol and water, two immiscible phases. A $\log \mathrm{P}$ value greater than 1 indicates that the substance preferentially separates into the hydrophobic phase, and thus is increasingly more lipophilic as the $\log \mathrm{P}$ value increases. ${ }^{94}$ To understand the polarity of the substance, the topological polar surface area (TPSA) can be explored. A TPSA of $\sim 90 \AA^{2}$ indicates a relatively polar structure that is unlikely to cross the blood brain barrier, while a TPSA $>140 \AA^{2}$ indicates a substance 
that is very polar and will not cross the blood brain barrier. ${ }^{7}$ These properties for the analytes in this work are summarized in Table 1.

Amphetamine (AMP) is a basic drug with an unsubstituted phenyl ring, a two-carbon side chain between the phenyl ring, and a primary amino group. It is the prototypical structure, seen in Table 1, of the phenethylamine class of drugs. The $\log$ P of AMP is 1.8, which indicates that it is relatively lipophilic in its neutral form. The $\mathrm{pK}_{\mathrm{a}}$ of AMP is 9.9, indicating that it is a basic drug that will be protonated at physiological $\mathrm{pH}$. The TPSA of amphetamine is $24 \AA^{2}$, indicating that the drug is somewhat polar, but not so much as to prevent passage across the blood brain barrier. Amphetamine is sparingly soluble in water, and aqueous solutions are alkaline to litmus paper. The chemical structure of AMP does not include an acidic function or an alkyl or benzyl functional group on the nitrogen. ${ }^{95}$

Cocaine (COC) is an amphoteric alkaloid consisting of a benzene ring and a cycloheptane ring connected via ester linkage. The cycloheptane ring has a nitrogen bridge between position 1 and position 5 and esters on positions 2 and 3 . The $\log \mathrm{P}$ of COC is 2.3 , indicating that it is a lipophilic substance in its neutral form. The $\mathrm{pK}_{\mathrm{a}}$ of the tertiary amine is 8.6. The TPSA is $56 \AA^{2}$, suggesting that the structure is somewhat polar, but still capable of crossing the blood brain barrier. Cocaine is sparingly soluble in water, and aqueous solutions are alkaline to litmus paper. The structure of COC does not include an acidic function, and it does include $N$-alkyl groups, as the amine acts as a bridge between carbon atoms in a cycloheptane ring. ${ }^{95}$

Morphine (MOR) is a pentacyclic opiate alkaloid with an oxygen bridge between position 4 and position 5 , phenolic groups at position 3 and position 6 , and a tertiary amine between position 9 and position 16. The $\log \mathrm{P}$ of MOR is 0.8 , suggesting that it is 
hydrophilic in its neutral form. The $\mathrm{pK}_{\mathrm{a}}$ of the tertiary amine is 8.02 and the $\mathrm{pK}_{\mathrm{a}}$ of the hydroxyl group on position 3 is 9.85 . The TPSA is $53 \AA^{2}$, suggesting that the structure is somewhat polar, but still capable of crossing the blood brain barrier. The $\mathrm{pH}$ of a saturated solution of MOR in water is 8.5 , indicating its alkalinity in aqueous solution. The structure includes an acidic hydroxyl group and alkyl substitution of the amino group. ${ }^{95}$

$\Delta^{9}$-Tetrahydrocannibinol (THC) is a tricyclic cannabinoid with a hydroxyl group on position 1, a five-carbon alkyl chain on position 3, two methyl substitutions at position 6, and a methyl group at position 9 . The $\log \mathrm{P}$ of THCis 7 , which indicates that it is very lipophilic. The $\mathrm{pK}_{\mathrm{a}}$ of the hydroxyl group is 10.6 . The structure includes an acidic function (hydroxyl); however it is largely a neutral, hydrophobic structure. The TPSA is $30 \AA^{2}$, indicating that the structure is relatively non-polar. The structure does not include an amino group..$^{95}$

Diazepam (DZP) is a benzodiazepine with a tertiary amine at position 1 and an imine at position 4, a ketone at position 2, and a chlorine at position 7. The $\log \mathrm{P}$ of DZP is 2.9, indicating that in its neutral state it is very lipophilic. The $\mathrm{pK}_{\mathrm{a}}$ is 3.4. The TPSA of DZP is $33 \AA^{2}$, indicating that is it relatively non-polar. The structure of DZP does not include acidic hydrogen atoms, but it does include alkyl substitution of the amine. ${ }^{95}$ 
Table 2: Structures and physiochemical properties of the drugs of abuse incorporated into blank hair.

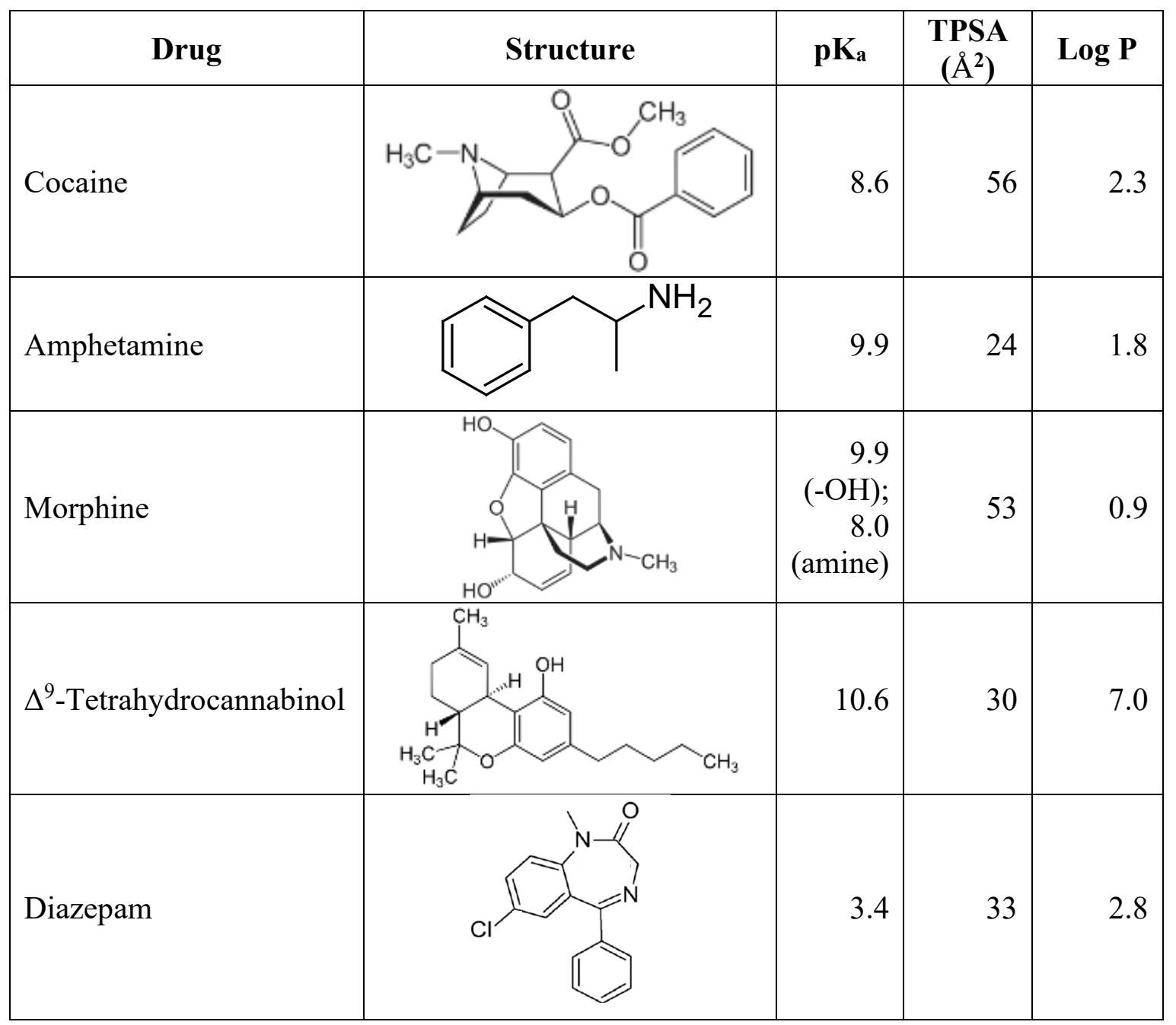




\section{PREPARATION OF INCORPORATED/ENTRAPPED, SINGLE ANALYTE HRM}

\subsection{Introduction}

Acquisition or preparation of HRM is essential for the development and validation of methodologies used in forensic hair analysis. There are generally three types of HRM: incorporated/entrapped HRM, externally contaminated HRM, and authentic HRM. Incorporated/entrapped HRM is prepared by exploiting the hydroscopic nature of hair to swell the cortex of the hair structure such that the scales of the cuticle are forced to move away from the one another, allowing the cortex to become exposed to the surrounding solution. Subsequently, any drug dissolved in the solution may enter the hair structure by means of passive diffusion. ${ }^{96}$ Externally contaminated HRM, which is used primarily for study of hair decontamination methods, has been prepared in a number of ways, but in general involves the application of drug to the surface of the hair structure either by rubbing the neat solid onto the hair or spraying a solution of the drug onto the hair. ${ }^{67,69,76}$ Authentic HRM is prepared by collecting specimen from individuals who self-reported as regular users of specific analytes and quantitating the self-reported substances in these specimen. At present, HRM containing selected xenobiotic substances are only available on a limited basis. Unfortunately, most xenobiotic substances relevant to forensic case work are not available as incorporated or authentic drug standards in human head hair. When HRM for a particular analyte is available for purchase, it is typically prepared using the same scheme as in this study, using an analyte-containing solvent mixture.

As previously discussed, a consensus has emerged that three primary incorporation mechanisms contribute to drug levels in hair. ${ }^{1,3,5,6,8,17,47,97-99}$ Exposure can be a result of drug-containing blood flow to the hair follicle, deposition of drug dissolved in sweat/sebum 
onto the hair shaft, and exposure to drugs from drug-containing aerosols in the environment. ${ }^{47}$ This knowledge is exploited to prepare HRM, where reference materials generally are prepared by soaking human head hair in xenobiotic-containing aqueous solution. The general concept of incorporation by exposure to a drug-containing solution is demonstrated in Figure 6. Mechanistically, this method most closely resembles the process of drug becoming incorporated in head hair after exposure to drug-containing sweat.

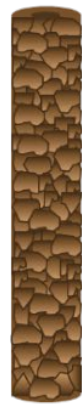

B

Aqueous solvent

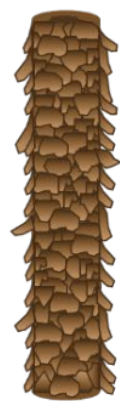

Addition of Drug Containing Solution to Aqueous Solvent

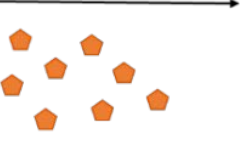

C

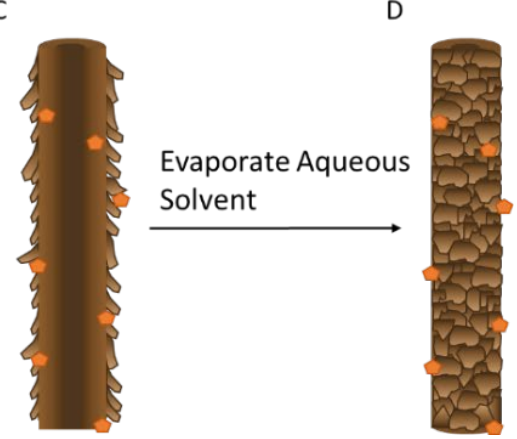

Figure 6: General Schematic of Incorporation Technique Employed to Prepare HRM. A: Drug-free hair is obtained. B: Drug-free hair is soaked in an aqueous solvent, causing the cuticle to swell and partially expose the cortex of the hair shaft. C (longitudinal crosssection): After spiking the aqueous solvent with the drug, simple diffusion allows drug to move between the swollen cuticle scales into the cortex of the hair. D: Aqueous solvent is evaporated, allowing the cuticle to smooth back over the cortex. This results in the incorporation of drug into the cortex of the hair and/or entrapment of the drug between cuticle scales.

The present work employed this incorporation technique to prepare HRM for amphetamine, cocaine, morphine, diazepam, and $\Delta^{9}$-tetrahydrocannabinol. It was hypothesized that the aqueous buffer system would need to be adjusted specifically for each substance to maximize the diffusion of drug into hair, as this process is predicted to have some dependence on the analyte's $\mathrm{pK}_{\mathrm{a}}$, lipophilicity, acid-base properties, chemical structure, and affinity to melanin. ${ }^{1,3,7,25,47}$ More polar compounds are less likely to be 
incorporated into hair because it is more difficult for these substances to cross cell membranes. Basic drugs are favorably incorporated into hair as a result of the $\mathrm{pH}$ gradient between blood $(\sim 7.4)$ and the cells in the hair follicle $(\sim 4.5-5.5))^{3,4,7,20}$ Additionally, protonated amino groups of basic drugs can interact with the free carboxyl groups on melanin. ${ }^{5,20}$ Additional factors noted to promote the incorporation of substances into hair are the presence of a nitrogen atom to facilitate association with melanin, the absence of an acidic function, and the presence of a long $N$-alkyl chain and/or $N$-substituted benzene ring. ${ }^{1,7}$

\subsection{Materials and Methods}

\subsubsection{Chemicals and Solvents}

Solvents and chemicals used for the preparation of mobile phases and sample preparation for LC-MS analysis (water, methanol, 2-propanol, dichloromethane, formic acid, ammonium formate) were of HPLC-grade and obtained from Fisher Scientific (Hampton, NH, USA).

Stock solutions of AMP, AMP-D11 DZP, HER, COC, DZP-D5, MOR-D3, and COCD3 were obtained at $1.0 \mathrm{mg} / \mathrm{mL}$ in methanol from Cayman Chemical (Ann Arbor, MI, USA). Solutions of $\Delta^{9}$-THC and $\Delta^{9}$-THC-D3 in acetonitrile, with concentrations of 10.0 and $1.0 \mathrm{mg} / \mathrm{mL}$, respectively, were purchased from Cayman Chemical. These stock solutions were used in the preparation of the wet chemistry-based incorporated HRM and calibration curves. 1,4-Dithiothreitol (DTT) was purchased from Sigma-Aldrich (St. Louis, MO, USA), and Proteinase-K was obtained from Invitrogen (Carlsbad, CA, USA). Three $\mathrm{kDa}$ molecular weight cut off PTFE spin filters were obtained from MilliporeSigma 
(Burlington, MA, USA). Single donor, unprocessed, natural black color human hair ( $\sim 300$

g) was obtained from a commercial source.

\subsubsection{HRM Production by Wet Chemistry-Based Incorporation}

Samples of human head hair $(800 \mathrm{mg})$ were soaked in $1 \mathrm{X}$ PBS, pH 6, spiked with drug stock solution at $1,500 \mathrm{pg} / \mathrm{mg}$ hair. Samples were incubated at $37^{\circ} \mathrm{C}$ while on an orbital shaker in a temperature-controlled oven. Aliquots of hair and spiked PBS solution were taken at $0,24,48,72,96$, and $120 \mathrm{~h}$ to monitor drug incorporation. The hair aliquots were washed three times with $1 \mathrm{X}$ PBS, $\mathrm{pH}$ 6, and once with 2-propanol for 1 min with agitation. Six aliquots of $30 \mathrm{mg}$ of washed hair were then pulverized using a Mini-BeadBeater 24 ball mill (Biospec; Bartlesville, OK, USA) at $3200 \mathrm{rpm}$ for $30 \mathrm{~s}$. Subsequently, three aliquots of pulverized hair was subjected to solvent extraction, followed by centrifugation, SPE, and LC-MS. The remaining three aliquots of hair were subjected to enzymatic extraction, followed by centrifugation, SPE, and LC-MS. Additionally, the spiked buffer solutions, extracted samples, and wash solutions were analyzed for their drug content by LC-MS. The total mass of hair was washed after incorporation with 3 volumes $(150 \mathrm{~mL}$ each) of 1X PBS followed by 1 volume of 2-propanol for 1 min each. The hair was dried at $40^{\circ} \mathrm{C}$ for $24 \mathrm{~h}$ in an oven, and then stored in aluminum foil at room temperature. This method was modified from Ropero-Miller, et al. ${ }^{96}$

Initially, small-scale incorporations were performed in triplicate at the $\mathrm{pK}_{\mathrm{a}}$ of the analyte, at two $\mathrm{pH}$ units above the $\mathrm{pK}_{\mathrm{a}}$, and at two $\mathrm{pH}$ units below the $\mathrm{pK}_{\mathrm{a}}$. The $\mathrm{pH}$ at which the incorporation of the analyte was highest was used for the large-scale, preparative 
incorporations, which were later used in the pre-treatment DoE studies. These methods can be understood in the schemes in Figure 7.

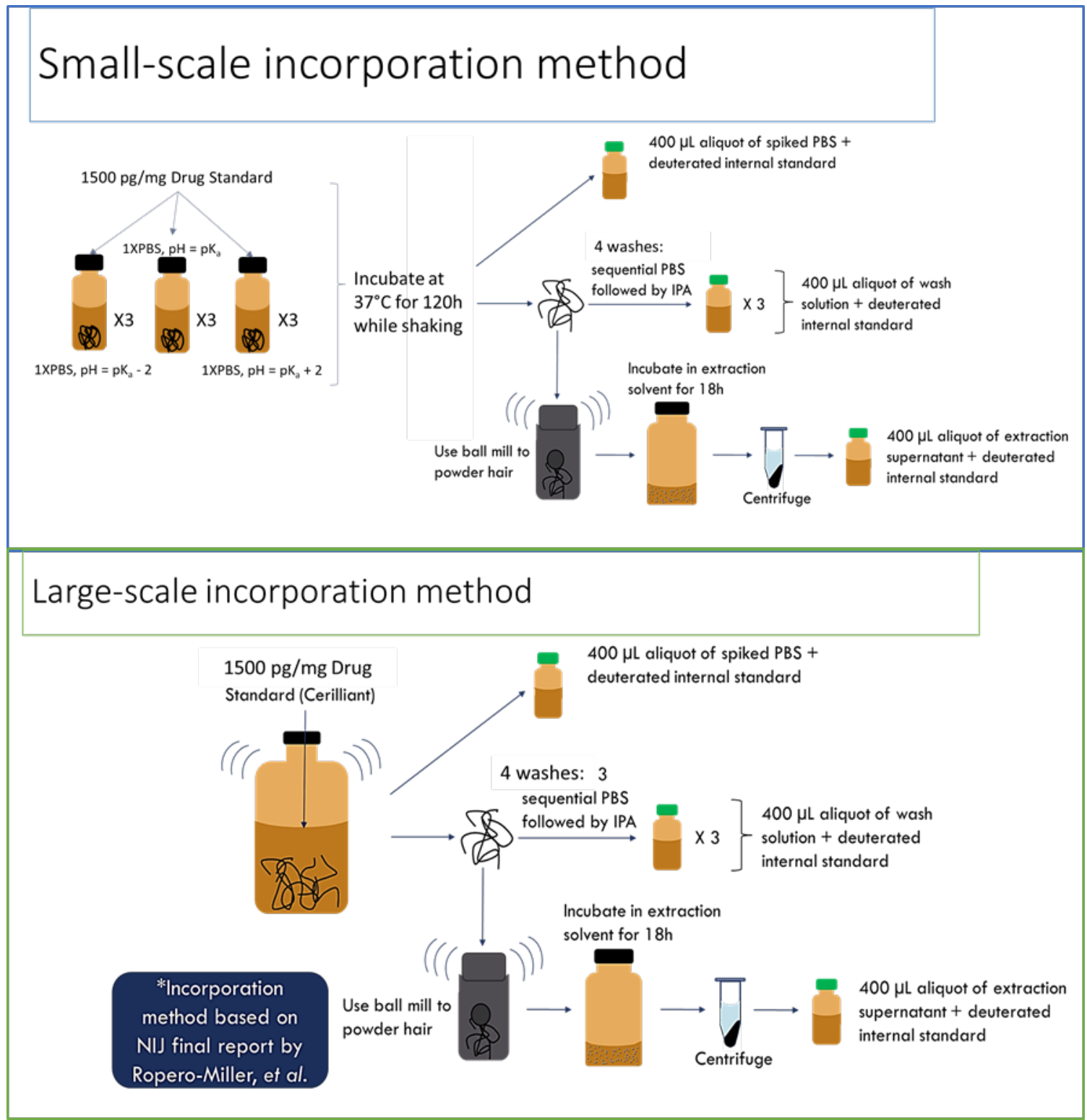

Figure 7: Depiction of small-scale and large-scale incorporation schemes.

\subsubsection{Isolation of Drugs from Human Head Hair}

Two extraction techniques were compared. Enzymatic degradation of the hair matrix was conducted by first incubating $30 \mathrm{mg}$ of hair with $12 \mathrm{mg} / \mathrm{mL}$ DTT for $2 \mathrm{~h}$ at $37^{\circ} \mathrm{C}$, then 
with $2 \mathrm{mg} / \mathrm{mL}$ proteinase $\mathrm{K}$ for $12 \mathrm{~h} \cdot{ }^{100}$ Solvent swelling of the hair matrix to allow for the extraction of drug held within the cortex was achieved by incubation of the hair in a mixture of methanol, acetonitrile, and $2 \mathrm{mM}$ ammonium formate $(25: 25: 50 \mathrm{v} / \mathrm{v})$ at $37{ }^{\circ} \mathrm{C} .{ }^{51}$ Samples were incubated for $24 \mathrm{~h}$ and subsequently centrifuged at $10,000 \mathrm{x}$ g to separate the hair particulates from the solution containing recovered drug. The supernatants from the enzymatically degraded samples were then centrifuged at $10,000 \times \mathrm{g}$ using a $3 \mathrm{kDa}$ molecular weight cut-off PTFE spin filter. The eluent solution was subjected to SPE cleanup and LC-MS analysis.

\subsubsection{Solid Phase Extraction}

For cleanup of hair extracts, Agilent Technologies (Santa Clara, CA, USA) Bond Elut Certify mixed mode cartridges were used following a method characterized for hair samples by Miller et al. ${ }^{101}$ The cartridges were installed on an SPE manifold with clean manifold liners. Under $3 \mathrm{~mm} \mathrm{Hg}$ vacuum, the cartridges were conditioned with two $1 \mathrm{~mL}$ volumes of methanol and then two $1 \mathrm{~mL}$ volumes of $1 \mathrm{X}$ phosphate buffered saline, $\mathrm{pH} 6$ (1X PBS). Next, the samples were spiked with a deuterated surrogate of the drug of interest, to serve as an internal standard. The samples were loaded to the cartridges at $1 \mathrm{~mL} / \mathrm{min}$ under $3 \mathrm{~mm} \mathrm{Hg}$ vacuum. The cartridges were then washed to eliminate matrix components with $1 \mathrm{~mL}$ of deionized water followed by $0.5 \mathrm{~mL}$ of $0.01 \mathrm{M}$ acetic acid. The cartridges were then dried under vacuum for $10 \mathrm{~min}$. A third wash was then conducted with $50 \mu \mathrm{L}$ of methanol, and the cartridges were dried again for 2 min. Elution of the compounds of interest was conducted by addition of $0.75 \mathrm{~mL}$ of ethyl acetate with $2 \%$ ammonium hydroxide at $1 \mathrm{~mL} / \mathrm{min}$ and subsequently with $0.75 \mathrm{~mL}$ of a mixture of dichloromethane, 
2-propanol, and 2\% aqueous ammonium hydroxide (78:20:2 v/v). The eluted samples were evaporated to dryness in a vacufuge for $1.5 \mathrm{~h}$ at $45^{\circ} \mathrm{C}$. Finally, the samples were reconstituted in $500 \mu \mathrm{L}$ of methanol for LC-MS analysis.

\subsubsection{HPLC-MS Analysis}

For drug analysis, $2 \mu \mathrm{L}$ of each isolate was injected onto a reversed-phase liquid chromatography column and subsequently analyzed by QqQ. The compounds were separated using an Agilent Technologies $1.8 \mu \mathrm{m}$ Zorbax Eclipse Plus $\mathrm{C}_{18}$ rapid resolution HD column $(2.1 \times 150 \mathrm{~mm} ; 1.8 \mu \mathrm{m})$. The aqueous mobile phase used was water with $0.1 \%$ formic acid and $5 \mathrm{mM}$ ammonium formate. The organic phase was $\mathrm{MeOH}$ with $0.1 \%$ formic acid. The separation was conducted using a gradient solvent system as follows: at 0 min the mobile phase was a $95 \%$ aqueous and $5 \%$ organic mixture, from 0 to $5 \mathrm{~min}$ the gradient adjusted to $100 \%$ organic mobile phase, from 5 to 8 min the gradient was held at $100 \%$ organic to flush the column. The mobile phase flow rate was $0.3 \mathrm{~mL} / \mathrm{min}$.

An LC-QqQ multiple reaction monitoring (MRM) method was used for the identification of analytes using an Agilent 6470 triple quadrupole mass spectrometer operated in positive ESI mode. The cycle time was $500 \mathrm{~ms}$. The cell accelerator voltage was $4 \mathrm{~V}$. The drying gas $\left(\mathrm{N}_{2}\right)$ was heated at $350^{\circ} \mathrm{C}$, and had a flow rate of $12 \mathrm{~L} / \mathrm{min}$. The sheath gas was also heated to $350^{\circ} \mathrm{C}$ and had a flow rate of $11 \mathrm{~mL} / \mathrm{min}$. The peaks were confirmed by MRM transitions corresponding to the integrated chromatographic peak. The MRM peaks were specified in the LC-MS method using the two most abundant transitions, one as a quantifier and the other as a qualifier. These transitions were identified by Agilent's MassHunter Optimizer program and are listed in Table 3. The data were acquired 
in Agilent MassHunter Qualitative software v.8. The spectral peak areas were integrated, and the data files were then imported into the MassHunter Quantitative software v. 7 for quantitation of the analytes.

Table 3: MRM transitions selected for LC -MS method.

\begin{tabular}{|c|c|c|}
\hline \multirow[t]{2}{*}{ Compound } & \multicolumn{2}{|c|}{ MRM Transition(s) } \\
\hline & $\begin{array}{l}\text { Precursor } \\
\text { Ion }(m / z)\end{array}$ & $\begin{array}{c}\text { Product } \\
\text { Ion }(m / z)\end{array}$ \\
\hline AMP & 136 & $\begin{array}{r}119 \\
91\end{array}$ \\
\hline AMP-D11 & 147 & $\begin{array}{r}130 \\
98\end{array}$ \\
\hline Cocaine & 304 & $\begin{array}{r}182 \\
82 \\
\end{array}$ \\
\hline Cocaine-D3 & 307 & $\begin{array}{r}185 \\
85\end{array}$ \\
\hline Diazepam & 285 & $\begin{array}{l}222 \\
193\end{array}$ \\
\hline Diazepam-D5 & 290 & $\begin{array}{l}198 \\
154 \\
\end{array}$ \\
\hline Morphine & 286 & $\begin{array}{l}201 \\
155 \\
\end{array}$ \\
\hline Morphine-D3 & 289 & $\begin{array}{r}126 \\
61 \\
\end{array}$ \\
\hline$\Delta^{9}-\mathrm{THC}$ & 315 & $\begin{array}{l}193 \\
123 \\
\end{array}$ \\
\hline$\Delta^{9}$-THC-D3 & 318 & $\begin{array}{r}196 \\
93\end{array}$ \\
\hline
\end{tabular}

A calibration curve was generated using drug standards with a $1 \mathrm{mg} / \mathrm{mL}$ concentration and the corresponding deuterated compound. The drug standards were diluted in $\mathrm{MeOH}$ to a final volume of $500 \mu \mathrm{L}$ to generate calibration solutions with $1,5,15,35,75,150,200$, and $250 \mathrm{ng} / \mathrm{mL}$ concentrations. Each calibrator also included $100 \mathrm{ng} / \mathrm{mL}$ of deuterated internal standard. 


\subsection{Results}

\subsubsection{Small-Scale Incorporations}

The results of the small-scale incorporations can be seen in Table 4 . The highest recoveries for $\mathrm{COC}$ and $\mathrm{THC}$ were observed at the $\mathrm{pK}_{\mathrm{a}}$ of the analytes, which are 8.6 and 10.6, respectively. There was no observable difference in the incorporation of MOR when the buffer was adjusted to different $\mathrm{pH}$. Large-scale incorporations were performed at the $\mathrm{pK}_{\mathrm{a}}$ of COC, MOR, and THC in accordance with these results. Small-scale incorporations were not performed for AMP and DZP.

Table 4: Average recovered drug from small-scale incorporated/entrapped HRM under various buffer conditions.

\begin{tabular}{|c|c|c|}
\hline Drug & $\begin{array}{c}\text { Incorporation } \\
\text { Condition }\end{array}$ & $\begin{array}{c}\text { Average amount of } \\
\text { Extracted Drug } \\
\text { (pg/mg) }\end{array}$ \\
\hline \multirow{3}{*}{ Cocaine } & $\mathrm{pH} 6.6$ & nd \\
\hline & $\mathrm{pH} 8.6$ & $40 \pm 24$ \\
\hline & pH 11.6 & nd \\
\hline \multirow{3}{*}{ Morphine } & $\mathrm{pH} 6.2$ & $2 \pm 0$ \\
\hline & $\mathrm{pH} 8.2$ & $2 \pm 0$ \\
\hline & $\mathrm{pH} 11.2$ & $2 \pm 0$ \\
\hline \multirow{4}{*}{$\Delta^{9}-\mathrm{THC}$} & $\mathrm{pH} 8.6$ & $24 \pm 10$ \\
\hline & $\mathrm{pH} 10.6$ & $30 \pm 5$ \\
\hline & $\mathrm{pH} 12.6$ & $10 \pm 8$ \\
\hline & $\begin{array}{l}90 \% 1 \mathrm{X} \mathrm{PBS}, \\
\mathrm{pH} 8: 10 \% \\
\mathrm{MeOH}\end{array}$ & $15 \pm 9$ \\
\hline
\end{tabular}

nd - none detected.

\subsubsection{Large-Scale Incorporations}

The various analytes were found to incorporate into natural black color human hair to different extents (Table 5). For AMP, 91\% of the drug spiked into the buffer was later extracted and detected after the incorporation was completed. The highest amount of 
incorporated and recovered drug, among the analytes studied, was detected for AMP with $1,369 \pm 414 \mathrm{pg} / \mathrm{mg}$ of drug detected. The next highest extent of incorporation and recovery of drug through extraction was observed for DZP; $315 \pm 51 \mathrm{pg} / \mathrm{mg}$ of drug was extracted from hair after incorporation, which accounted for $21 \%$ of the original $1,500 \mathrm{pg} / \mathrm{mg}$ spiked into the incorporation buffer. THC, MOR and COC had low levels of incorporation and subsequent extraction. The amount of THC extracted from incorporated hair was $118 \pm 29$ $\mathrm{pg} / \mathrm{mg}$, which accounted for only $8 \%$ of the drug originally spiked into solution for incorporation. The extent of incorporation of MOR and COC was $<5 \%$.

Table 5: Average recovered drug from large-scale incorporation/entrapment of drugs of abuse in HRM.

\begin{tabular}{|c|c|c|c|}
\hline Drug & $\begin{array}{l}\text { Spiked } \\
\text { (pg/mg) }\end{array}$ & $\begin{array}{c}\text { Extracted } \\
(\mathbf{p g} / \mathbf{m g})\end{array}$ & $\%$ Incorporated \\
\hline Amphetamine & \multirow{5}{*}{1,500} & $1,369 \pm 414$ & 91 \\
\hline Diazepam & & $315 \pm 51$ & 21 \\
\hline$\Delta^{9}-\mathrm{THC}$ & & $118 \pm 29$ & 8 \\
\hline Morphine & & $78 \pm 34$ & 5 \\
\hline Cocaine & & $57 \pm 21$ & 4 \\
\hline
\end{tabular}

\subsection{Discussion}

The amount of drug that was incorporated into the HRM was some portion of a known concentration that was intentionally added to the buffer solution used to swell the cuticle of the hair. The drug under study was then able to enter the hair strand by passive diffusion. Subsequently, the amount of drug that became incorporated was analyzed, and the material served as a reference for authentic specimen in subsequent experiments.

The ability of some compounds to associate more readily with hair has been hypothesized to be influenced by a number of factors, including the lipophilicity, $\mathrm{pK}_{\mathrm{a}}$, 
structure, size, protein binding capacity, and melanin affinity, with each drug having unique mechanisms by which it is held in hair. ${ }^{47}$ Incorporation of substances into hair following either systemic or direct external exposure may be due to covalent or ionic binding between hair components and the compounds of interest, van der Waals forces, hydrophobic interactions, or a combination thereof. It has been demonstrated in numerous studies that drugs can associate with melanin. . $^{2,4103,104,11,18,20-22,47,99,102}$ The nature of these associations is not well understood. However, it has been suggested that basic, lipophilic drugs may have a greater affinity for binding/interacting with melanin. ${ }^{3}$

The physical and chemical properties of the drugs under study in the present research are different, which likely contributes to the differences in their extent of incorporation. Amphetamine had the highest levels of incorporation by swelling the cuticle of the hair and allowing for passive diffusion of the drug from the solution into the hair. Amphetamine is the only drug included in this study that has a primary amine, which may allow for ionic, covalent, or polar interactions between AMP and the carboxylic acid moieties of eumelanin, a hypothesis that will be discussed more fully in Chapter 6 . The next most extensive incorporation was observed for DZP. The structure of DZP is such that there is a benzene ring on a rotatable bond, which may allow the drug to more freely participate in $\pi-\pi$ interactions with the aromatic portions of melanins. Finally, THC was observed to incorporate at lower, but still detectable amounts. The low incorporation of THC may be a result of the high lipophilicity of the drug, which would allow for the association with lipophilic regions in keratin and keratin-associated proteins and/or the 18-methyl eicosanoic acid in the cell membrane complex of the cuticle of the hair. Cocaine and MOR did not incorporate extensively into hair by means of passive diffusion. Morphine is a 
relatively large, multi-cyclic drug with ionizable hydroxyl moieties and a tertiary amine. The low incorporation of MOR may be a result of its large, relatively polar structure. Cocaine is an amphoteric drug with an ionizable tertiary amine. The TPSA of COC is relatively high, indicating that it is somewhat polar, which like MOR, may be the cause of its low incorporation into hair. Additionally, variation in the incorporation of different analytes under different $\mathrm{pH}$ conditions may be a result of differences in the charge state of the drug and/or components of hair.

Ropero-Miller et al. were able to incorporate AMP, COC, MOR and THC using the same method employed in the present work. While the amount of AMP incorporated in the present work was comparable to concentrations observed by Ropero-Miller et al. with the same, relatively high, variability, COC, THC, and MOR were incorporated at much lower concentrations in the present work than previously reported. ${ }^{96}$ The high levels of variability were attributed to using whole strands of hair, which limits the surface area of the cortex, where drug is believed to interact with hair components for their subsequent retention in the material, exposed to the incorporation buffer. ${ }^{96}$ These authors were also able to incorporate MOR in multi-analyte HRM; in the present study, MOR was incorporated at low amounts with high variability. ${ }^{96}$ These differences may be a result of differences in diffusion rates of MOR into hair when multiple analytes are in solution, and in the RoperMiller et al. method, when compared to a single analyte solution, as was used in this work. To date, incorporates/entrapped HRM containing DZP has not been reported. RoperoMiller et al. also reported that high variability in concentration in the incorporated/entrapped HRM lead to analytical uncertainty. ${ }^{96}$ While 
incorporated/entrapped HRM is still available, it has become less available as authentic HRM use for method development and validation has become favorable.

Using incorporated HRM for DoE studies has both benefits and limitations. The greatest advantage of incorporated HRM over other types of reference material, such as authentic user hair, is that it can be prepared to contain either single analytes or multiple drugs with adequate concentrations for subsequent extraction and quantitation, as was demonstrated by Ropero-Miller et al. ${ }^{96}$ The concentration of AMP incorporated in the present study was consistent with that reported by Ropero-Miller et al. using the same incorporation method. However, incorporation of COC, MOR, and THC was significantly less than reported previously. For DZP, previous reports of reference concentrations following incorporation are unavailable. ${ }^{96}$ A major limitation of using incorporated HRM is that the exact mechanism by which drugs are held within the hair matrix is not yet fully understood; therefore drug incorporated into hair by means of diffusion from a buffer solution may not perfectly represent drug incorporated by means of blood flow to the hair follicle, as in authentic specimens.

Finally, it is important to consider the applicability of these reference materials, as they do not necessarily represent authentic specimens. Amphetamine and COC are two of the most widely studied drugs of abuse in hair, and it is well known that both incorporate into hair readily after systemic use of the drug. ${ }^{90,92}$ Here it was seen that AMP incorporated extensively by means of swelling the cuticle of the hair away from the cortex with a mixture of aqueous and organic solvents, but $\mathrm{COC}$ did not incorporate well by these means. Nevertheless, these incorporated HRM did allow for a proof of concept study of the pre- 
treatment and extraction parameters in a controlled manner using the DoE approach outlined in Chapter 4.

\subsection{Conclusions}

Incorporated/entrapped HRM was successfully prepared for use in subsequent experiments for AMP, THC, and DZP. The extent of incorporation of MOR and COC was too low to for repeatable experimental results, and thus not carried into the DoE studies. The low incorporation rates of MOR and COC is likely due to their relatively high polarity as compared to the other analytes in this study. 


\section{SYSTEMATIC COMAPRISON OF HAIR DECONTAMINATION PARAMETERS}

\section{VIA $2^{4}$ FRACTIONAL FACTORIAL BLOCK DESIGN}

\subsection{Introduction}

One of the greatest challenges and sources of uncertainty in the toxicological analysis of hair involves decontamination prior to extraction and analysis. The purpose of decontamination is to remove oils, lipids, and cosmetic products and to eliminate any analyte that may be superficially associated with the surface of the hair as a result of contact with contaminated surfaces or drug vapors and residues that may exist in the environment of the individual being sampled. ${ }^{69}$ Important considerations for the decontamination of hair samples include the choice of solvent/solution used to wash the hair, the volume of that solvent/solution used in relation to the amount of hair being decontaminated, and the length of exposure of the hair to these solvents/solutions. ${ }^{69,70}$

Removal of external contamination from hair is not necessarily a simple process. For example, a recent study by Cuypers et al. reported that aqueous wash procedures, rather than removing surface contaminated drug, can actually move it deeper into the hair matrix. ${ }^{66}$ A decontamination procedure for a lipophilic drug, such as THC, may be ineffective for polar drugs such as cocaine. ${ }^{105}$ The SoHT has endorsed the use of an aqueous wash step followed by an organic wash. ${ }^{38}$ However, these recommendations are general, and thus a large number of analyte-specific wash procedures have emerged with a variety of effectiveness. ${ }^{35,74,76,101,105-108}$

Studies have been conducted to compare some of the more commonly employed techniques for decontamination as complete protocols or individual variables involved in the wash protocol. For example, Stout et al. compared decontamination protocols 
originally reported by Cairnes et al. and Romano et al. to study their efficacy for removing COC from the surface of hair, finding that neither method was sufficient for removing all of the surface contamination, a result consistent with the findings of the original authors of each technique. ${ }^{69,72,74}$ Ropero-Miller et al. and Morris-Kukoski et al. also observed that the Cairnes et al. decontamination protocol was insufficient for removing COC from the surface of hair. ${ }^{106,109}$ Duvivier et al. compared complete protocols for the decontamination of THC, finding that a multi-step wash with a combination of methanol and SDS was most effective. ${ }^{76}$ Mantineiks et al. compared the efficacy of several solvents in regard to removing COC and methamphetamine contamination, finding again that none resulted in the complete removal of the drug. ${ }^{75}$

Although, as described above, reports do exist that compare selected hair decontamination procedures for specific drugs, to date there has been no comprehensive, statistically rigorous, side-by-side evaluation of decontamination approaches for multiple classes of drugs. Considering the lack of systematic comparative analysis of decontamination protocols for a wider set of commonly abused drugs, the present study employed a DoE approach to assess a limited set of variables in the decontamination process for AMP, DZP, THC, COC, and HER. Instead of including MOR in this study, HER was chosen as it is a commonly smoked drug, where MOR is more likely to be injected intravenously $;{ }^{95}$ as discussed in Chapter 1 , smoked drugs pose a greater risk for external contamination than drugs that are introduced via different routes of exposure. These drugs were chosen because they pose high potential for external contamination. Powders can be aerosolized and deposited on hair; HER, COC, DZP, and AMP are often purchased by users as powders or pulverized into a powder by users before use. ${ }^{48,95}$ Another 
means by which drugs are aerosolized and subsequently may cause external contamination is through smoking, as is a common route of administration of THC. ${ }^{95}$

As an example of DoE, a $2^{4}$ fractional factorial design allows for the determination of the effects of four factors, each with two levels (high and low), and the combinations of these factors on the dependent variable. The factors are denoted with upper-case letters (A, B, C, D, etc.). Each factor has a high and a low level. The $2^{\mathrm{k}}$ notation refers to the number of combinations of factor levels, with $k$ being the number of factors under study. These combinations of levels are called design points. Each design point is denoted with lower case letters, such that if the letter is listed, the factor is held at the high level in the design point. Since none of the columns of the main factors (A, B, C, D, etc.) in the design are exactly the same nor opposite, the columns are said to be linearly independent, allowing for the ANOVA to be solved as a linear model.

Often, there are factors in an experiment that are known to have a predictable effect on the dependent variable, but these factors are not of interest, therefore they are called nuisance factors (also known as blocking factors). When only one replicate of each design point is available, it is impossible to observe all interactions of factors. By using nuisance factors to block the design points, the unobservable interactions can be controlled. The design intentionally allows the block to be completely the same or opposite of only one higher order interaction (i.e., 2, 3, 4, etc.-factor interactions); the two are said to be completely confounded, meaning that ANOVA will not be able to separate the variance in the data set resulting from the block from the variance that is resulting from the confounded interaction. Thus, we cannot observe the direct effect of the block nor the interaction on the dependent variable. The term which is allowed to be completely confounded with the 
block is called the defining contrast. The defining contrast is chosen by predicting which interactions may not be significant or of interest and is usually chosen to be a higher level interaction. $^{79}$

In $2^{\mathrm{k}}$ factorial designs, such as the one chosen for this work (Table 5), each factor of interest has 2 levels (i.e., high and low) for $k$ number of factors. Therefore, there are $2^{\mathrm{k}}$ combinations of factor levels, which make up the list of design points to be studied. ${ }^{79}$ If the factor is listed in the design point, then that factor is held at the high level; if it is not listed, then it is at the low level. As an example, design point "bc" had the high levels of factor B and factor $\mathrm{C}$ applied to it; while factor $\mathrm{A}$ and factor $\mathrm{D}$ were at the low levels (Table 6). 
Table 6: Augmented $2^{4}$ fractional factorial block design for comparison of decontamination parameters.

\begin{tabular}{|c|c|c|c|c|c|c|c|c|c|c|c|c|c|c|c|c|c|c|}
\hline Block $(1,2)$ & Design Point & A & B & C & D & $A B$ & $A C$ & BC & $A D$ & BD & CD & ABD & ACD & BCD & $A B C$ & $A B C D$ & Block 1 & Block 2 \\
\hline \multirow{4}{*}{ (low, low) } & 1 & - & - & - & - & + & + & + & + & + & + & - & - & - & - & + & even & even \\
\hline & bc & - & + & + & - & - & - & + & + & - & - & + & + & - & - & + & even & even \\
\hline & $a d b$ & + & + & - & + & + & - & - & + & + & - & + & - & - & - & - & even & even \\
\hline & acd & + & - & + & + & - & + & - & + & - & + & - & + & - & - & - & even & even \\
\hline \multirow{4}{*}{ (low, high) } & ac & + & - & + & - & - & + & - & - & + & - & + & - & + & + & + & even & odd \\
\hline & $a b$ & + & + & - & - & + & - & - & - & - & + & - & + & + & + & + & even & odd \\
\hline & bcd & - & + & + & + & - & - & + & - & + & + & - & - & + & + & - & even & odd \\
\hline & d & - & - & - & + & + & + & + & - & - & - & + & + & + & + & - & even & odd \\
\hline \multirow{4}{*}{ (high, low) } & bd & - & + & - & + & - & + & - & - & + & - & - & + & - & - & + & odd & even \\
\hline & $\mathrm{cd}$ & - & - & + & + & + & - & - & - & - & + & + & - & - & - & + & odd & even \\
\hline & a & + & - & - & - & - & - & + & - & + & + & + & + & - & - & - & odd & even \\
\hline & $a b c$ & + & + & + & - & + & + & + & - & - & - & - & - & - & - & - & odd & even \\
\hline \multirow{4}{*}{ (high, high) } & b & - & + & - & - & - & + & - & + & - & + & + & - & + & + & - & odd & odd \\
\hline & c & - & - & + & - & + & - & - & + & + & - & - & + & + & + & - & odd & odd \\
\hline & ad & + & - & - & + & - & - & + & + & - & - & - & - & + & + & + & odd & odd \\
\hline & abcd & + & + & + & + & + & + & + & + & + & + & + & + & + & + & + & odd & odd \\
\hline
\end{tabular}


In an augmented design, the interaction effects are determined by considering the product of the individual factors. Using design point "bc" as the example again, the interaction effect for $\mathrm{AB}$ is determined by understanding the product of $\mathrm{A} \cdot \mathrm{B}$. In this case, $\mathrm{A}$ is low and B is high. When a negative is multiplied by a positive, the result is negative, which is indicated in the $\mathrm{AB}$ interaction column for design point "bc."

However, some interaction effects are not observable. Generally, in an augmented design, no two columns are the exact same or exact opposite of one another, which means the factors are linearly independent, thus a linear model, such as ANOVA, may be used to analyze the variance in the resultant data set. ${ }^{79}$ In Table 6, the columns for "AD," "BCD," "ABC," and "block" contradict this rule and are said to be completely confounded as a result. These columns are said to be completely confounded with each other, and thus the variance in the dataset attributed to these factors cannot be understood with the $2^{4}$ fractional factorial block design. These confounding factors are also called the defining contrasts and are chosen within the block design.

When a $2^{\mathrm{k}}$ design is broken into $2^{\mathrm{m}}$ blocks, the blocks are of the size $2^{k} / 2^{m}$ or $2^{\mathrm{k}-\mathrm{m}}$, where $\mathrm{m}$ is the number of blocking factors. Each blocking factor has 2 levels. Each blocking factor has a corresponding defining contrast, with which it is completely confounded. These defining contrasts are usually chosen to be high-level interactions, i.e., three- and four-factor interactions. Once selected, design points are designated as odd or even, depending on the number of letters in common with the chosen defining contrast, distinguishing their level within each block. ${ }^{79}$ In the specific design chosen for the present work, $\mathrm{ABC}$ was chosen as the defining contrast for block $1, \mathrm{BCD}$ was chosen as the defining 
contrast for block 2, and as a result $\mathrm{AD}$ was completely confounded with the interaction effect between block 1 and 2 .

To date, only limited work using multivariate statistical approaches such as DoE to compare protocols associated with the pre-treatment of hair has been reported. Those efforts were focused on examining selected pre-treatment parameters for analysis of the ethanol consumption biomarker ethyl glucuronide in hair. ${ }^{50,110}$ The present chapter describes the application of DoE in the systematic comparison of pre-treatment parameters for a variety of drugs of abuse. Separate DoE studies were conducted for decontamination protocols and for all other pre-treatment parameters, including two approaches for final drug extraction from the hair matrix, which will be described in Chapter V. In the decontamination study, a $2^{4}$ fractional factorial block design together with ANOVA and post-hoc testing were employed with the hypothesis that this approach would demonstrate the potential utility of DoE to facilitate optimization of pre-treatment parameters in forensic hair analysis.

\subsection{Materials and Methods}

\subsubsection{Chemicals and Solvents}

Solvents and chemicals used for the preparation of mobile phases and sample preparation for LC-MS analysis (water, methanol, 2-propanol, dichloromethane, formic acid, ammonium formate) were of HPLC-grade and obtained from Fisher Scientific (Hampton, NH, USA). 
Stock solutions of AMP, AMP-D11 DZP, HER, COC, DZP-D5, MOR-D3, and COCD3 were obtained at $1.0 \mathrm{mg} / \mathrm{mL}$ in methanol from Cayman Chemical (Ann Arbor, MI, USA). Solutions of $\Delta^{9}$-THC and $\Delta^{9}$-THC-D3 in acetonitrile at 10.0 and $1.0 \mathrm{mg} / \mathrm{mL}$ concentrations, respectively, were purchased from Cayman Chemical. These stock solutions were used in the preparation of calibration curves. Neat solids of D-AMP sulfate, DZP, HER, and COC for intentional contamination of hair were purchased from Cayman Chemical (Ann Arbor, MI, USA). Solids of 1,4-Dithiothreitol (DTT) and Proteinase-K were purchased from Sigma-Aldrich (St. Louis, MO, USA) Invitrogen (Carlsbad, CA, USA), respectively. Three kDa molecular weight cut off PTFE spin filters were obtained from MilliporeSigma (Burlington, MA, USA). Single donor, unprocessed, natural black color human hair $(\sim 300 \mathrm{~g})$ was obtained from a commercial source.

\subsection{2 $\quad 2^{4}$ Fractional Factorial Design for Comparing Decontamination Protocols}

A $2^{4}$ fractional factorial block design was implemented to understand how the parameters listed in Table 7 affect the recovery of drug intentionally applied to blank hair under different decontamination protocols. 
Table 7: Definitions of factors and levels of these factors under study in the $2^{4}$ fractional factorial block design.

\begin{tabular}{|c|c|c|}
\hline Factor & Decontamination Design & \\
\hline \multirow{2}{*}{ A } & \multirow{2}{*}{$\begin{array}{c}\text { Aqueous decontamination } \\
\text { solvent }\end{array}$} & $(+) 1 \% \mathrm{SDS}$ \\
\hline & & (-) HPLC water \\
\hline \multirow{2}{*}{$\mathrm{B}$} & \multirow{2}{*}{$\begin{array}{c}\text { Organic decontamination } \\
\text { solvent }\end{array}$} & $(+)$ dichloromethane \\
\hline & & (-) methanol \\
\hline \multirow{2}{*}{$\mathrm{C}$} & \multirow{2}{*}{$\begin{array}{c}\text { Number of consecutive } \\
\text { aqueous washes }\end{array}$} & $(+) 3$ \\
\hline & & $(-) 1$ \\
\hline \multirow{2}{*}{$\mathrm{D}$} & \multirow{2}{*}{$\begin{array}{c}\text { Number of consecutive } \\
\text { organic washes }\end{array}$} & $(+) 3$ \\
\hline & & $(-) 1$ \\
\hline \multirow{2}{*}{ Block 1} & \multirow{2}{*}{ Sequence of washes } & $(+)$ Organic before aqueous \\
\hline & & (-) Aqueous before organic \\
\hline \multirow{2}{*}{ Block 2} & \multirow{2}{*}{ Wash time } & (+) $30 \mathrm{~min}$ \\
\hline & & $(-) 30 \mathrm{~s}$ \\
\hline
\end{tabular}

The application of these parameters to hair samples was dictated by the design matrix (Table 6) and is represented visually in Figure 8. Initially, $30 \mathrm{mg}$ aliquots of blank hair were externally contaminated with $1 \mathrm{mg}$ of neat solid drug for each replicate $(\mathrm{n}=3)$ of each design point when analyzing for DZP, HER, and COC. The hair and neat solid drug were added to steel milling jars and shaken in a Mini-BeadBeater 24 ball mill (Biospec; Bartlesville, OK, USA), without milling beads, for $30 \mathrm{~s}$ at 3,200 rpm to homogeneously coat the hair with drug. Contaminated hair strands were then removed from the milling jar and transferred to a fresh container for subsequent procedures. The application of THC was performed by pipetting $1 \mathrm{~mL}$ of a $1 \mathrm{mg} / \mathrm{mL}$ solution of drug in acetonitrile onto a $30 \mathrm{mg}$ aliquot of hair. Following vortexing, solvent was evaporated in an Eppendorf Vacufuge Plus (Hauppauge, NY, USA) under the aqueous vacuum conditions at $45^{\circ} \mathrm{C}$ for $45 \mathrm{~min}$. Samples were decontaminated by adding $1 \mathrm{~mL}$ of the decontamination solvent/solution and 
agitating for $1 \mathrm{~min}$ on an orbital shaker, as specified in the design matrix (Table 6). The hair samples were then dried overnight.

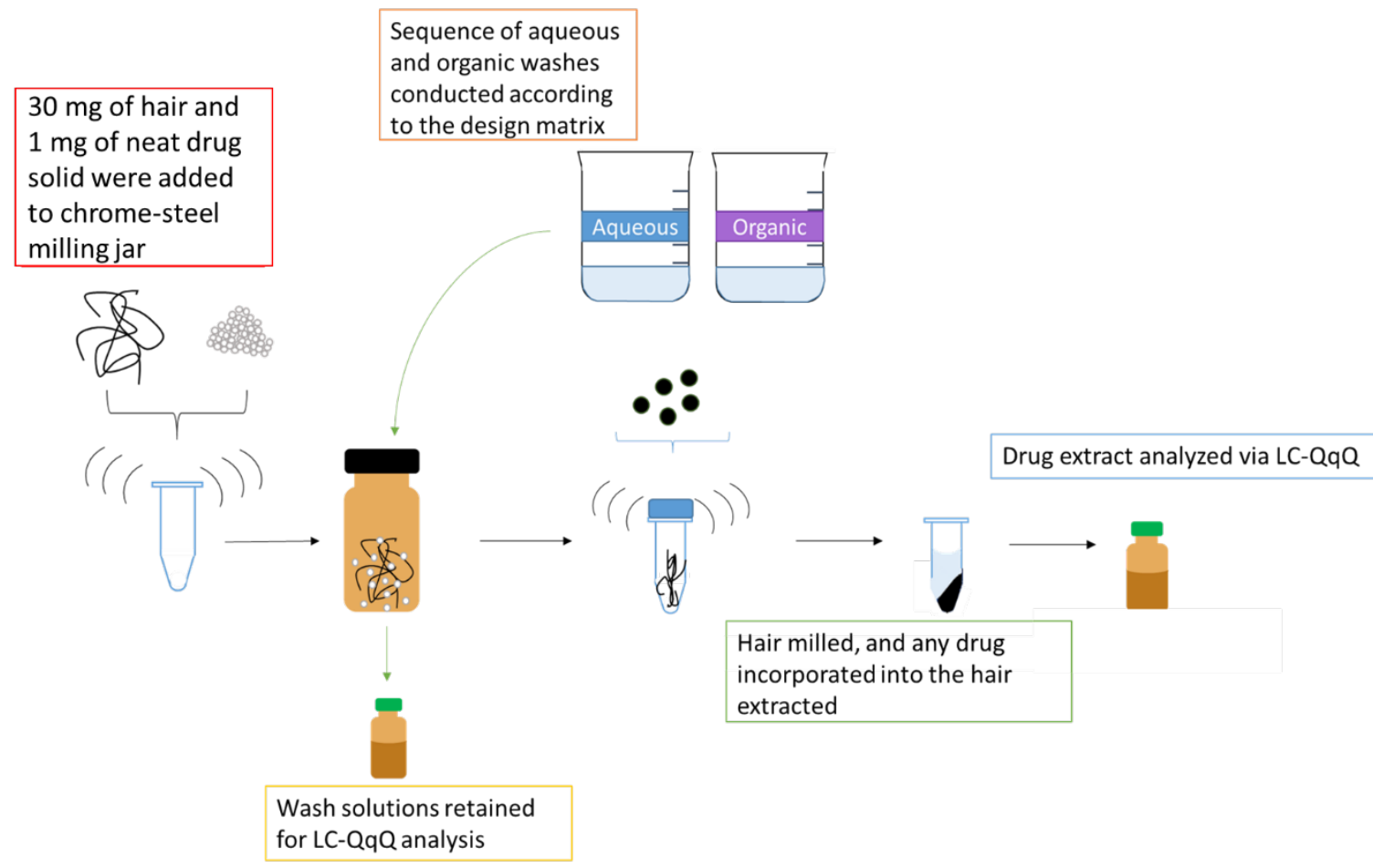

Figure 8: Analytical scheme for sample preparation for the decontamination DoE studies.

Drug that may have been incorporated deeper into the hair matrix was also determined following extraction by means of enzymatic degradation of the matrix. Extracted samples and wash solutions were purified by spin filter and SPE before LC-MS analysis, as previously described and analysis performed by high performance liquid chromatography coupled to triple quadrupole mass spectrometry (HPLC-QqQ). The MRM transitions used for quantitative analysis of are listed in Table 8. A randomized list of design points was used to determine the order in which samples were prepared and analyzed, with three replicate samples for each design point. Quantitation of the drug detected in each sample allowed for subsequent statistical analysis by ANOVA F-Test and Tukey's HSD to 
determine which, if any, parameters or combination(s) of parameters resulted in significant differences in the recovery of drug from the washes. Drug recovery from wash solutions was calculated by summing the amount of drug detected in the sequential washes and dividing by the difference in the amount of drug left in the milling jar after transfer from the initial mass of drug initially applied to the hair.

Table 8: MRM transitions used for identification and quantitation of analytes via HPLCMS.

\begin{tabular}{|l|c|c|}
\hline \multirow{2}{*}{ Compound Name } & \multicolumn{2}{|c|}{ MRM Transition(s) } \\
\cline { 2 - 3 } Cocaine & Precursor Ion $(\boldsymbol{m} / \mathbf{z})$ & Product Ion $(\boldsymbol{m} / \mathbf{z})$ \\
\hline \multirow{2}{*}{ Cocaine-D3 } & 304 & $\begin{array}{c}182 \\
82\end{array}$ \\
\hline \multirow{2}{*}{ Diazepam } & 307 & $\begin{array}{c}185 \\
85\end{array}$ \\
\hline \multirow{2}{*}{ Diazepam-D5 } & 285 & 222 \\
& 290 & 193 \\
\hline \multirow{2}{*}{ Heroin } & \multirow{2}{*}{370} & 154 \\
\hline \multirow{2}{*}{ Morphine-D3 } & 289 & 211 \\
\hline \multirow{2}{*}{$\Delta^{9}$-THC } & \multirow{2}{*}{315} & 165 \\
\hline \multirow{2}{*}{$\Delta^{9}$-THC-D3 } & \multirow{2}{*}{318} & 126 \\
\hline
\end{tabular}

\subsubsection{Statistical Analysis}

The LC -MS analysis allowed for the quantification of drug from all of the samples analyzed. The quantity of drug detected in the triplicate extracts, using the technique with the highest recovery, was then employed to find the statistical parameters necessary to complete an ANOVA table comparing the parameters under study in the DoE matrix. If a 
significant difference was observed between treatment groups (i.e., design points) by means of the ANOVA F-test, Tukey's HSD post-hoc analysis was conducted to determine which parameters or combinations of parameters resulted in this difference. JMP software $\left(13^{\text {th }}\right.$ ed.) was used for all statistical analyses.

\subsection{Results}

Figure 9 shows the plots of the percent recovery of each analyte for each design point in triplicate. In general, a limited number of design points for each analyte were found to produce the highest total recovery of drug in the wash solutions. The parameters that were applied to the samples within these treatment groups were tabulated to draw conclusions about the most effective decontamination protocols for each analyte. The similarities between design points with the highest recoveries were used to make a consensus statement about which of the two levels of each parameter was most effective for removing each of the analytes from the surface of hair. 

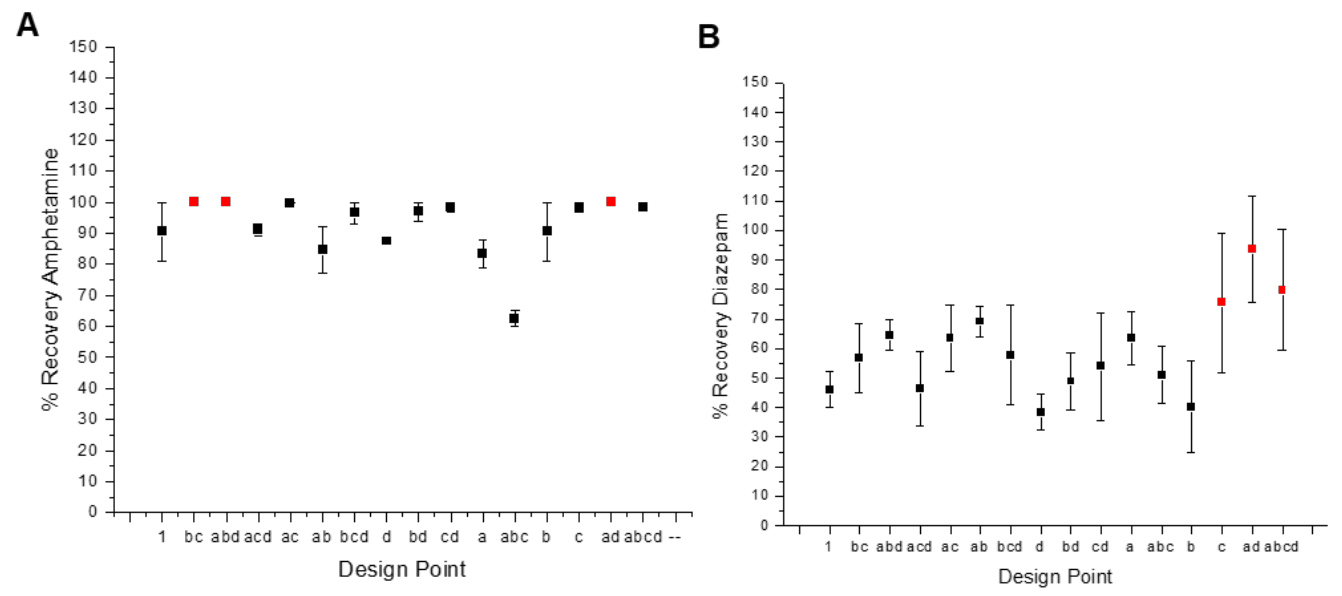

C
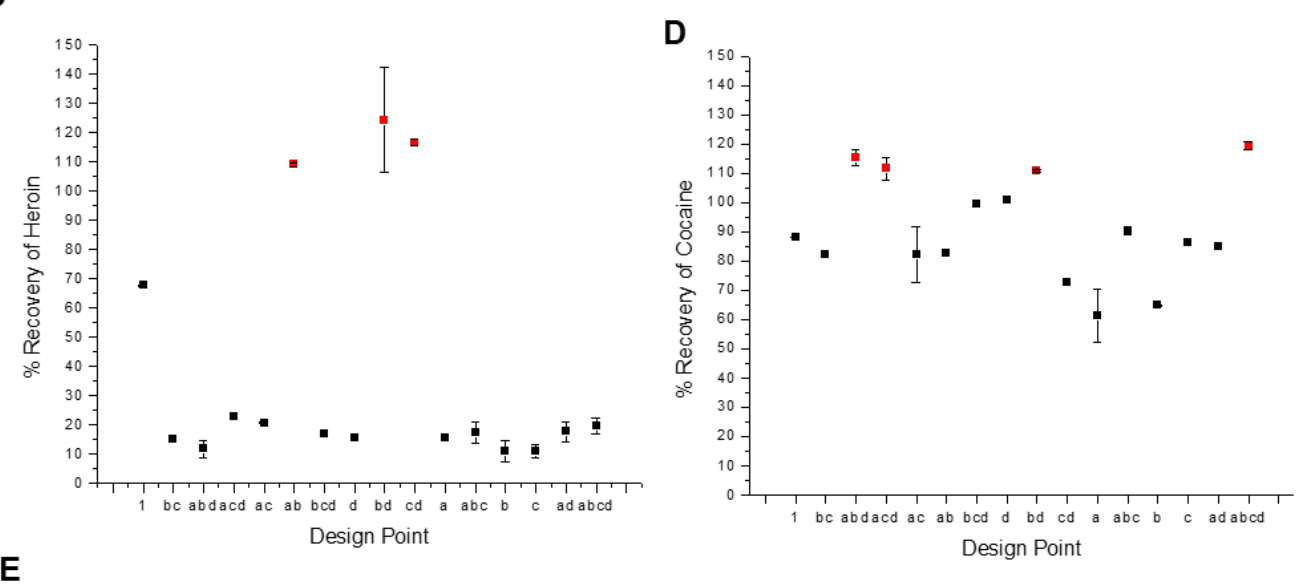

E

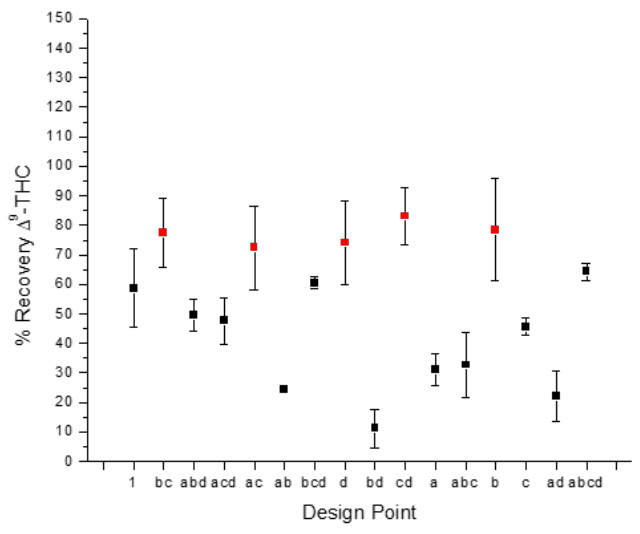

Figure 9: Plot of percent recovery of (A) AMP, (B) DZP, (C) HER, (D) COC, and (E) $\Delta^{9}$-THC detected in the wash solutions for each design point $(n=3)$. The $y$-axis is the percent recovery of the drug; the x-axis is the design point. Points highlighted in red represent design points with highest recovery. Parameters associated with each design point are summarized in Table 6. 
The ANOVA comparison of the factors and combinations of factors (Table 9) in removal of externally contaminated AMP from hair was conducted, resulting in p-values less than the $\alpha$-level (0.05) for all factors except B (particle size of homogenized hair) and $\mathrm{C}$ (aqueous decontamination solvent).

Table 9: ANOVA for $2^{4}$ fractional factorial block design comparing decontamination parameters for $A M P$.

\begin{tabular}{|c|c|c|c|c|c|}
\hline Source & $\begin{array}{l}\text { Sum of } \\
\text { Squares }\end{array}$ & $\begin{array}{l}\text { Degrees } \\
\text { Freedom }\end{array}$ & Mean Squares & F Ratio & $\begin{array}{c}\text { p- } \\
\text { value }\end{array}$ \\
\hline $\mathrm{A}$ & 0.45 & 1 & $4.54 \times 10^{-1}$ & 1.81 & 0.19 \\
\hline $\mathrm{B}$ & 0.11 & 1 & $1.13 \times 10^{-1}$ & 0.45 & 0.51 \\
\hline $\mathrm{C}$ & 0.01 & 1 & $7.00 \times 10^{-3}$ & 0.03 & 0.86 \\
\hline $\mathrm{D}$ & 0.33 & 1 & $3.34 \times 10^{-1}$ & 1.33 & 0.26 \\
\hline $\mathrm{AB}$ & 0.63 & 1 & $6.32 \times 10^{-1}$ & 2.52 & 0.38 \\
\hline $\mathrm{AC}$ & 0.65 & 1 & $6.48 \times 10^{-1}$ & 2.58 & 0.12 \\
\hline $\mathrm{BD}$ & 0.59 & 1 & $5.92 \times 10^{-1}$ & 2.36 & 0.14 \\
\hline $\mathrm{CD}$ & 0.47 & 1 & $4.68 \times 10^{-1}$ & 1.87 & 0.18 \\
\hline $\mathrm{BC}$ & 0.13 & 1 & $1.26 \times 10^{-1}$ & 0.50 & 0.48 \\
\hline CDA & 1.27 & 1 & 1.27 & 5.05 & 0.03 \\
\hline DAB & 1.16 & 1 & 1.16 & 4.62 & 0.04 \\
\hline $\mathrm{ABCD}$ & 1.58 & 1 & 1.58 & 6.32 & 0.02 \\
\hline Number of organic washes & 0.06 & 1 & $5.75 \times 10^{-2}$ & 0.23 & 0.63 \\
\hline Number of aqueous washes & 0.00 & 1 & $4.06 \times 10^{-3}$ & 0.02 & 0.88 \\
\hline error & 4.51 & 18 & $2.51 \times 10^{-1}$ & & \\
\hline total & 5.16 & 32 & $1.61 \times 10^{-1}$ & & \\
\hline
\end{tabular}

Figure 9A shows the recovery of AMP in the combined aqueous and organic washes for each design point. Average recovery was highest for design points ad, bc, and abd at $100 \%$. The extracts of these samples were analyzed for AMP content, all indicating concentrations below the limit of detection. The conditions under which recovery was highest are summarized in Table 10. Design points ad and abd shared several decontamination conditions including the aqueous decontamination solvent ( $1 \% \mathrm{SDS})$, the 
number of aqueous and organic washes applied to the samples ( 1 and 3, respectively), the order in which the washes were conducted (aqueous before organic), and the length of washes (30 s). Design points bc and abd were similar in that they both employed dichloromethane as the organic decontamination solvent.

Table 10: List of parameters for the design points resulting in the highest recovery of AMP.

\begin{tabular}{|l|c|c|c|}
\hline \multirow{2}{*}{\multicolumn{1}{c|}{ Wash Parameter }} & \multicolumn{3}{c|}{ Design Point } \\
\cline { 2 - 4 } & ad & bc & abd \\
\hline A: Aqueous decontamination solvent & $1 \% \mathrm{SDS}$ & HPLC water & $1 \% \mathrm{SDS}$ \\
\hline B: Organic decontamination solvent & $\mathrm{MeOH}$ & DCM & DCM \\
\hline C: Number of aqueous washes & 1 & 3 & 1 \\
\hline D: Number of organic washes & 3 & 1 & 3 \\
\hline Block 1: Sequence of washes & Aqueous first & Organic first & Aqueous first \\
\hline Block 2: Wash time & $30 \mathrm{~s}$ & $30 \mathrm{~min}$ & $30 \mathrm{~s}$ \\
\hline Drug Recovered in Washes & $100 \pm 5 \%$ & $100 \pm 8 \%$ & $100 \pm 12 \%$ \\
\hline
\end{tabular}

The ANOVA F-Test results for the decontamination of DZP (Table 11) indicated that significant individual and combinatorial factors (i.e. p-value $<\alpha$-level, 0.05) included aqueous decontamination solvent/solution (A), the combinatorial effect of aqueous solvent/solution and the organic decontamination solvent (AB), the combinatorial effect between aqueous solvent/solution and number of consecutive aqueous washes (AC), the combinatorial effect of organic solvent and number of organic washes (BD), the combinatorial effect of aqueous solvent/solution, organic solvent, and number of consecutive aqueous washes (ACD), the combinatorial effect of aqueous solvent/solution, organic decontamination solvent, and number of consecutive organic washes (ABD), and the combinatorial effect of all main factors (ABCD). 
Table 11: ANOVA for $2^{4}$ fractional factorial block design comparing decontamination parameters for DZP.

\begin{tabular}{|l|r|r|c|r|c|}
\hline \multicolumn{1}{|c|}{ Source } & $\begin{array}{c}\text { Sum of } \\
\text { Squares }\end{array}$ & $\begin{array}{c}\text { Degrees } \\
\text { Freedom }\end{array}$ & $\begin{array}{c}\text { Mean } \\
\text { Squares }\end{array}$ & F Ratio & p-value \\
\hline A & 2416.16 & 1 & $2.42 \times 10^{3}$ & 8.61 & 0.006139 \\
\hline B & 29.15 & 1 & $2.91 \times 10^{1}$ & 0.10 & 0.753883 \\
\hline C & 76.07 & 1 & $7.61 \times 10^{1}$ & 0.27 & 0.606908 \\
\hline D & 59.34 & 1 & $5.93 \times 10^{1}$ & 0.21 & 0.649864 \\
\hline AB & 3211.56 & 1 & $3.21 \times 10^{3}$ & 11.44 & 0.001911 \\
\hline AC & 5223.76 & 1 & $5.22 \times 10^{3}$ & 18.62 & 0.000143 \\
\hline BD & 1329.12 & 1 & $1.33 \times 10^{3}$ & 4.74 & 0.036956 \\
\hline CD & 366.39 & 1 & $3.66 \times 10^{2}$ & 1.31 & 0.260884 \\
\hline BC & 223.27 & 1 & $2.23 \times 10^{2}$ & 0.80 & 0.377772 \\
\hline CDA & 6134.08 & 1 & $6.13 \times 10^{3}$ & 21.86 & 0.000051 \\
\hline DAB & 3741.10 & 1 & $3.74 \times 10^{3}$ & 13.33 & 0.000923 \\
\hline ABCD & 10121.44 & 1 & $1.01 \times 10^{4}$ & 36.07 & $<0.00001$ \\
\hline $\begin{array}{l}\text { Number of organic } \\
\text { washes }\end{array}$ & 770.01 & 1 & $7.07 \times 10^{2}$ & 2.74 & 0.107641 \\
\hline $\begin{array}{l}\text { Number of aqueous } \\
\text { washes }\end{array}$ & 1406.87 & 1 & $1.41 \times 10^{3}$ & 5.01 & 0.032287 \\
\hline error & 8979.51 & 32 & $2.81 \times 10^{2}$ & & \\
\hline total & 19100.95 & 47 & $4.06 \times 10^{2}$ & & \\
\hline
\end{tabular}

Recoveries of DZP in wash solutions ranged from $40 \pm 15 \%$ to $94 \pm 18 \%$, with the highest recoveries noted for design points $\mathrm{c}$, ad, and abcd (Figure 9B). Decontamination parameters for these optimal design points are summarized in Table 12. Blocking factors 1 and 2 were just the same for all of the samples in the treatment groups associated with these design points, employing the organic wash before the aqueous wash and decontaminating for 30 min during each wash step. Design points ad and abcd had in common the use of $1 \%$ SDS as the aqueous decontamination solution. Additionally, three sequential organic washes were applied to samples for design points ad and abcd. Design points $\mathrm{c}$ and ad had in common the use of methanol as the organic decontamination solvent. 
Design points $\mathrm{c}$ and abcd both utilized three consecutive washes with the aqueous decontamination solvent/solution.

Table 12: List of parameters for the design points resulting in the highest recovery of DZP.

\begin{tabular}{|l|c|c|c|}
\hline \multirow{2}{*}{\multicolumn{1}{c|}{ Wash Parameter }} & \multicolumn{3}{c|}{ Design Point } \\
\cline { 2 - 4 } & $\mathbf{c}$ & ad & abcd \\
\hline A: Aqueous decontamination solvent & $\mathrm{HPLC}$ Water & $1 \%$ SDS & $1 \%$ SDS \\
\hline B: Organic decontamination solvent & $\mathrm{MeOH}$ & $\mathrm{MeOH}$ & $\mathrm{DCM}$ \\
\hline C: Number of aqueous washes & 3 & 1 & 3 \\
\hline D: Number of organic washes & 1 & 3 & 3 \\
\hline Block 1: Sequence of washes & Organic first & Organic first & Organic first \\
\hline Block 2: Wash time & $30 \mathrm{~min}$ & $30 \mathrm{~min}$ & $30 \mathrm{~min}$ \\
\hline Drug Recovered in Washes & $76 \pm 24 \%$ & $94 \pm 18 \%$ & $80 \pm 20 \%$ \\
\hline
\end{tabular}

The ANOVA F-Test results for the decontamination of HER (Table 13) indicated that all individual factors and combinations of factors resulted in p-values less than the $\alpha$-level $(0.05)$ and thus were significant. 
Table 13: ANOVA for $2^{4}$ fractional factorial block design comparing decontamination parameters for HER.

\begin{tabular}{|l|r|r|c|r|l|}
\hline \multicolumn{1}{|c|}{ Source } & $\begin{array}{c}\text { Sum of } \\
\text { Squares }\end{array}$ & $\begin{array}{c}\text { Degrees } \\
\text { Freedom }\end{array}$ & $\begin{array}{c}\text { Mean } \\
\text { Squares }\end{array}$ & F Ratio & p-value \\
\hline A & 3885.39 & 1 & $3.89 \times 10^{3}$ & 107.65 & $<0.00001$ \\
\hline B & 273.60 & 1 & $2.74 \times 10^{2}$ & 7.58 & $<0.00001$ \\
\hline C & 3276.92 & 1 & $3.28 \times 10^{3}$ & 90.79 & $<0.00001$ \\
\hline D & 1135.55 & 1 & $1.14 \times 10^{3}$ & 31.46 & $<0.00001$ \\
\hline AB & 8668.40 & 1 & $8.67 \times 10^{3}$ & 240.17 & $<0.00001$ \\
\hline AC & 7203.75 & 1 & $7.20 \times 10^{3}$ & 199.59 & $<0.00001$ \\
\hline BD & 28098.67 & 1 & $2.81 \times 10^{4}$ & 778.52 & $<0.00001$ \\
\hline CD & 8388.59 & 1 & $8.39 \times 10^{3}$ & 232.42 & $<0.00001$ \\
\hline BC & 14513.94 & 1 & $1.45 \times 10^{4}$ & 402.13 & $<0.00001$ \\
\hline CDA & 25506.01 & 1 & $2.55 \times 10^{4}$ & 706.69 & $<0.00001$ \\
\hline DAB & 26032.72 & 1 & $2.60 \times 10^{4}$ & 721.28 & $<0.00001$ \\
\hline ABCD & 76318.13 & 1 & $7.63 \times 10^{4}$ & 2114.53 & $<0.00001$ \\
\hline $\begin{array}{l}\text { Number of organic } \\
\text { washes }\end{array}$ & 533.91 & 1 & $5.34 \times 10^{2}$ & 14.79 & $<0.00001$ \\
\hline $\begin{array}{l}\text { Number of aqueous } \\
\text { washes }\end{array}$ & 5439.08 & 1 & $5.44 \times 10^{3}$ & 150.70 & $<0.00001$ \\
\hline error & 1154.95 & 32 & $3.61 \times 10^{1}$ & & \\
\hline total & 77473.08 & 47 & $1.65 \times 10^{3}$ & & \\
\hline
\end{tabular}

Recoveries of HER in wash solutions ranged from $12 \pm 3 \%$ _t $124 \pm 18 \%$, with design points ab, bd, and cd demonstrating the highest total recoveries of HER (Figure 9C). Decontamination parameters for these design points are summarized in Table 14. While the variation within these design points is high, it is clear that they resulted in the highest recoveries of HER and allowed for the common variables to be noted for design points bd and cd included HPLC water as the aqueous decontamination solvent, three consecutive organic washes before the aqueous wash, and decontamination for $30 \mathrm{~s}$ during each wash step. Commonalities between design points $a b$ and bd included DCM as the decontamination solvent and only one aqueous wash applied to the hair. 
Table 14: List of parameters for the design points resulting in the highest recovery of HER.

\begin{tabular}{|l|c|c|c|}
\hline \multirow{2}{*}{\multicolumn{1}{|c|}{ Wash Parameter }} & \multicolumn{3}{c|}{ Design Point } \\
\cline { 2 - 4 } & ab & bd & cd \\
\hline A: Aqueous decontamination solvent & $1 \% \mathrm{SDS}$ & HPLC water & HPLC water \\
\hline B: Organic decontamination solvent & DCM & DCM & MeOH \\
\hline C: Number of aqueous washes & 1 & 1 & 3 \\
\hline D: Number of organic washes & 1 & 3 & 3 \\
\hline Block 1: Sequence of washes & Aqueous first & Organic first & Organic first \\
\hline Block 2: Wash time & 30 min & $30 \mathrm{~s}$ & $30 \mathrm{~s}$ \\
\hline Drug Recovered in Washes & $109 \pm 1 \%$ & $124 \pm 18 \%$ & $116 \pm 1 \%$ \\
\hline
\end{tabular}

The ANOVA F-Test results for the decontamination of COC (Table 15) indicated that all individual factors and combinations of factors resulted in p-values less than the $\alpha$-level (0.05), and therefore had a significant effect.

Table 15: ANOVA for $2^{4}$ fractional factorial block design comparing decontamination parameters for $C O C$.

\begin{tabular}{|l|r|r|c|r|c|}
\hline \multicolumn{1}{|c|}{ Source } & $\begin{array}{c}\text { Sum of } \\
\text { Squares }\end{array}$ & $\begin{array}{c}\text { Degrees } \\
\text { Freedom }\end{array}$ & $\begin{array}{c}\text { Mean } \\
\text { Squares }\end{array}$ & F Ratio & p-value \\
\hline A & 331.70 & 1 & $3.32 \times 10^{2}$ & 17.93 & $<0.00001$ \\
\hline B & 1120.58 & 1 & $1.12 \times 10^{3}$ & 60.56 & $<0.00001$ \\
\hline C & 237.46 & 1 & $2.37 \times 10^{2}$ & 12.83 & $<0.00001$ \\
\hline D & 5854.62 & 1 & $5.85 \times 10^{3}$ & 316.38 & $<0.00001$ \\
\hline AB & 3316.10 & 1 & $3.32 \times 10^{3}$ & 179.20 & $<0.00001$ \\
\hline AC & 1867.88 & 1 & $1.87 \times 10^{3}$ & 100.94 & $<0.00001$ \\
\hline BD & 7967.76 & 1 & $7.97 \times 10^{3}$ & 430.58 & $<0.00001$ \\
\hline CD & 6622.32 & 1 & $6.62 \times 10^{3}$ & 357.87 & $<0.00001$ \\
\hline BC & 1358.04 & 1 & $1.36 \times 10^{3}$ & 73.39 & $<0.00001$ \\
\hline CDA & 1341.63 & 1 & $1.34 \times 10^{3}$ & 72.50 & $<0.00001$ \\
\hline DAB & 10046.67 & 1 & $1.00 \times 10^{4}$ & 542.92 & $<0.00001$ \\
\hline ABCD & 13754.42 & 1 & $1.38 \times 10^{4}$ & 743.28 & $<0.00001$ \\
\hline $\begin{array}{l}\text { Number of organic } \\
\text { washes }\end{array}$ & 979.19 & 1 & $9.79 \times 10^{2}$ & 52.91 & $<0.00001$ \\
\hline $\begin{array}{l}\text { Number of aqueous } \\
\text { washes }\end{array}$ & 27.76 & 1 & $2.78 \times 10^{1}$ & 1.50 & 0.10108 \\
\hline error & 592.16 & 32 & $1.85 \times 10^{1}$ & & \\
\hline total & 14346.58 & 47 & $3.05 \times 10^{2}$ & & \\
\hline
\end{tabular}


Recoveries of COC in wash solutions ranged from $61 \pm 9 \%$ to $119 \pm 1 \%$, with the highest recoveries observed for design points bd, abd, acd, and abcd (Figure 9D). Decontamination parameters for these design points are summarized in Table 19. Each of these design points employed three consecutive organic washes. Design points bd, abd, and acd each used a single aqueous decontamination wash for $30 \mathrm{~s}$. The aqueous decontamination solution applied to samples in treatment groups abd, acd, and abcd was $1 \%$ SDS. The organic decontamination solvent applied to samples in treatment groups bd, abd, and abcd was DCM. Design points abd and acd represented treatment with the aqueous decontamination solvent/solution first, whereas bd and abcd utilized organic solvent first.

Table 16: List of parameters for the design points resulting in the highest recovery of COC.

\begin{tabular}{|l|c|c|c|c|}
\hline \multirow{2}{*}{ Wash Parameter } & \multicolumn{4}{c|}{ Design Point } \\
\cline { 2 - 5 } & bd & abd & acd & abcd \\
\hline $\begin{array}{l}\text { A: Aqueous decontamination } \\
\text { solvent }\end{array}$ & $\begin{array}{c}\text { HPLC } \\
\text { water }\end{array}$ & $1 \%$ SDS & $1 \%$ SDS & $1 \%$ SDS \\
\hline $\begin{array}{l}\text { B: Organic decontamination } \\
\text { solvent }\end{array}$ & DCM & DCM & MeOH & DCM \\
\hline C: Number of aqueous washes & 1 & 1 & 1 & 3 \\
\hline D: Number of organic washes & 3 & 3 & 3 & 3 \\
\hline Block 1: Sequence of washes & $\begin{array}{c}\text { Organic } \\
\text { first }\end{array}$ & $\begin{array}{c}\text { Aqueous } \\
\text { first }\end{array}$ & $\begin{array}{c}\text { Aqueous } \\
\text { first }\end{array}$ & $\begin{array}{c}\text { Organic } \\
\text { first }\end{array}$ \\
\hline Block 2: Wash time & $30 \mathrm{~s}$ & $30 \mathrm{~s}$ & $30 \mathrm{~s}$ & $30 \mathrm{~min}$ \\
\hline Drug Recovered in Washes & $111 \pm 1 \%$ & $115 \pm 3 \%$ & $112 \pm 4 \%$ & $119 \pm 1 \%$ \\
\hline
\end{tabular}

The ANOVA F-Test results for decontamination of THC (Table 17) indicated that individual factors $\mathrm{A}$ and $\mathrm{B}$, along with all combinations of factors, resulted in p-values less than the $\alpha$-level (0.05). 
Table 17: ANOVA for $2^{4}$ fractional factorial block design comparing decontamination parameters for THC.

\begin{tabular}{|l|r|r|r|r|r|}
\hline \multicolumn{1}{|c|}{ Source } & $\begin{array}{c}\text { Sum of } \\
\text { Squares }\end{array}$ & $\begin{array}{c}\text { Degrees } \\
\text { Freedom }\end{array}$ & $\begin{array}{c}\text { Mean } \\
\text { Squares }\end{array}$ & F Ratio & p-value \\
\hline A & 2623 & 1 & 2623 & 15 & 0.00135 \\
\hline B & 171 & 1 & 171 & 1 & 0.33220 \\
\hline C & 2262 & 1 & 2262 & 13 & 0.00237 \\
\hline D & 9 & 1 & 9 & 0 & 0.82151 \\
\hline AB & 2914 & 1 & 2914 & 17 & 0.00080 \\
\hline AC & 5147 & 1 & 5147 & 30 & 0.00005 \\
\hline BD & 3761 & 1 & 3761 & 22 & 0.00025 \\
\hline CD & 2767 & 1 & 2767 & 16 & 0.00103 \\
\hline BC & 2444 & 1 & 2444 & 14 & 0.00178 \\
\hline CDA & 6867 & 1 & 6867 & 40 & 0.00001 \\
\hline DAB & 10056 & 1 & 10056 & 59 & $<0.00001$ \\
\hline ABCD & 978 & 1 & 978 & 6 & 0.02170 \\
\hline Number of organic washes & 318 & 1 & 318 & 2 & 0.17646 \\
\hline Number of aqueous washes & 1149 & 1 & 1149 & 7 & 0.01762 \\
\hline error & 2742 & 16 & 171 & & \\
\hline total & 18391 & 31 & 593 & & \\
\hline
\end{tabular}

Recoveries of THC in wash solutions ranged from $22 \pm 9 \%$ to $83 \pm 10 \%$, with highest recoveries detected for design points bc, ac, $d, c d$, and b (Figure 9E). Decontamination parameters for these design points are summarized in Table 18. Design points bc, d, cd, and $\mathrm{b}$ utilized HPLC water as the aqueous decontamination solvent, and design points bc, ac, and d included application of the aqueous wash prior to the organic. Design points bc, ac, and cd had in common the use of three consecutive aqueous washes, while design points bc, ac, and b all employed a single organic wash. A 30 min was utilized for design points ac, $d$, and $b$. 
Table 18: List of parameters for the design points resulting in the highest recovery of $\Delta^{9}$ THC.

\begin{tabular}{|c|c|c|c|c|c|}
\hline \multirow[b]{2}{*}{ Wash Parameter } & \multicolumn{5}{|c|}{ Design Point } \\
\hline & bc & ac & d & cd & $\mathbf{b}$ \\
\hline $\begin{array}{l}\text { A: Aqueous } \\
\text { decontamination } \\
\text { solvent }\end{array}$ & $\begin{array}{l}\text { HPLC } \\
\text { Water }\end{array}$ & $1 \% \mathrm{SDS}$ & $\begin{array}{l}\text { HPLC } \\
\text { Water }\end{array}$ & $\begin{array}{l}\text { HPLC } \\
\text { Water }\end{array}$ & $\begin{array}{l}\text { HPLC } \\
\text { Water }\end{array}$ \\
\hline $\begin{array}{l}\text { B: Organic } \\
\text { decontamination } \\
\text { solvent }\end{array}$ & DCM & $\mathrm{MeOH}$ & $\mathrm{MeOH}$ & $\mathrm{MeOH}$ & DCM \\
\hline $\begin{array}{l}\text { C: Number of } \\
\text { aqueous washes }\end{array}$ & 3 & 3 & 1 & 3 & 1 \\
\hline $\begin{array}{l}\text { D: Number of } \\
\text { organic washes }\end{array}$ & 1 & 1 & 3 & 3 & 1 \\
\hline $\begin{array}{l}\text { Block 1: Sequence } \\
\text { of washes }\end{array}$ & $\begin{array}{l}\text { Aqueous } \\
\text { first }\end{array}$ & $\begin{array}{l}\text { Aqueous } \\
\text { first }\end{array}$ & $\begin{array}{l}\text { Aqueous } \\
\text { first }\end{array}$ & $\begin{array}{l}\text { Organic } \\
\text { first }\end{array}$ & $\begin{array}{l}\text { Organic } \\
\text { first }\end{array}$ \\
\hline Block 2: Wash time & $30 \mathrm{~s}$ & $30 \mathrm{~min}$ & $30 \mathrm{~min}$ & $30 \mathrm{~s}$ & $30 \mathrm{~min}$ \\
\hline $\begin{array}{l}\text { Recovered Drug in } \\
\text { Wash }\end{array}$ & $77 \pm 11 \%$ & $72 \pm 14 \%$ & $74 \pm 14 \%$ & $83 \pm 9 \%$ & $78 \pm 17 \%$ \\
\hline
\end{tabular}

Consensus parameters that resulted in the highest recovery for each analyte are summarized in Table 19. These data indicate that the optimal combination of decontamination parameters was different for each individual drug. 
Table 19: Summary of the parameters resulting in the highest average recovery of analytes.

\begin{tabular}{|l|c|c|c|c|c|}
\hline \multicolumn{1}{|c|}{ Wash Parameter } & AMP & DZP & $\Delta^{\mathbf{9}}$-THC & COC & HER \\
\hline $\begin{array}{l}\text { A: Aqueous } \\
\text { decontamination solvent }\end{array}$ & $1 \%$ SDS & $1 \%$ SDS & $\begin{array}{c}\text { HPLC } \\
\text { Water }\end{array}$ & $1 \%$ SDS & $\begin{array}{c}\text { HPLC } \\
\text { Water }\end{array}$ \\
\hline $\begin{array}{l}\text { B: Organic } \\
\text { decontamination solvent }\end{array}$ & 1 & 3 & 3 & 1 & DCM \\
\hline $\begin{array}{l}\text { C: Number of aqueous } \\
\text { washes }\end{array}$ & 3 & 3 & 1 & 3 & 3 \\
\hline $\begin{array}{l}\text { D: Number of organic } \\
\text { washes }\end{array}$ & $\begin{array}{c}\text { Aqueous } \\
\text { first }\end{array}$ & $\begin{array}{c}\text { Organic } \\
\text { first }\end{array}$ & $\begin{array}{c}\text { Aqueous } \\
\text { first }\end{array}$ & $\begin{array}{c}\text { Aqueous } \\
\text { first }\end{array}$ & $\begin{array}{c}\text { Organic } \\
\text { first }\end{array}$ \\
\hline $\begin{array}{l}\text { Block 1: Sequence of } \\
\text { washes }\end{array}$ & $30 \mathrm{~s}$ & $30 \mathrm{~min}$ & $30 \mathrm{~min}$ & $30 \mathrm{~s}$ & $30 \mathrm{~s}$ \\
\hline Block 2: Wash time & & &
\end{tabular}

\subsection{Discussion}

In the present study, a DoE matrix combined with ANOVA F-test analysis was employed to systematically assess the effects of two levels of four different independent decontamination parameters on recovery of four physically and chemically diverse drugs from externally contaminated hair. Unlike previous studies, the DoE approach allowed for the determination of multiple significant factors impacting the decontamination of each analyte in a single experiment. In addition, DoE allowed the determination of the effects of combinations of decontamination factors (e.g., solvents, wash times, order of washes, etc.), a capability that facilitates the study of the impact of changing multiple variables within the experiment. The present report is the first in which multiple parameters in hair decontamination were simultaneously compared for a variety of drugs. Results of the ANOVA F-Test for COC and HER showed that all the individual sources (i.e., A, B, C, and D) were significant. In contrast, for DZP and THC, only one or two of the individual sources, respectively, were significant. Perhaps more importantly, higher-level interactions 
(e.g., $\mathrm{AB}, \mathrm{ABC}$, etc.) were significant for all four drugs, indicating that combinations of the variables had a significant effect on the level of drug detected in the wash solutions.

There are several previous reports that compared wash protocols following SoHT guidelines. ${ }^{75,76,111}$ These studies concluded that methanol was most effective organic solvent at removing surface contamination, while $1 \%$ SDS was the most effective aqueous decontamination solution for the removal of a variety of analytes..$^{75,76,111}$ Some previous observations are in contrast to findings reported herein. For example, in the present study, DCM was found to be more effective than methanol at removing $\mathrm{COC}$ from the hair surface. Other work has shown that, for removal of THC contamination, SDS was the most effective aqueous solvent/solution, while methanol, DCM, and chloroform were effective organic solvents. ${ }^{76}$ In contrast, in the present study it was found that water and methanol were the most effective solvents for removing THC from hair. Some of these inconsistencies may be attributable to differences in experimental approach, primarily regarding the contamination process; other studies have used a drug-containing solution of synthetic sweat or have burned marijuana in a chamber that contained hair to contaminate its surface with THC. ${ }^{67,105}$ Additionally, it may not be valid to directly compare results from hair decontamination studies using a "one factor at a time" approach to those generated using a multivariate technique such as DoE.

There are numerous methods that are commonly referenced for decontamination of hair samples prior to toxicological analysis. For example, Wang and Cone reported a decontamination technique that called for submersion of the hair samples in methanol for $\sim 1$ min. ${ }^{112}$ A method in which hair was vortexed in DCM three times for 5 min each was reported by Kintz. ${ }^{113}$ Another method reported initially by Cairnes et al. included first 
vigorously agitating hair in $n$-propanol for $15 \mathrm{~min}$ at $37^{\circ} \mathrm{C}$, then in $0.01 \mathrm{M} \mathrm{PBS}$ containing $0.01 \%$ bovine serum albumin, $\mathrm{pH} 6$, twice for 30 min each, and finally with the same buffer twice for 60 min each. ${ }^{72}$ A more complex method, reported by Baumgartner and Hill, included staining with methylene blue to assess the porosity of the hair sample, followed by washing with ethanol for $15 \mathrm{~min}$ at $37^{\circ} \mathrm{C}$ with shaking. More porous samples were then washed with a mixture of ethanol and water. Non-porous and slightly porous hair were washed with $0.01 \mathrm{M} \mathrm{PBS,} \mathrm{pH} \mathrm{5.6;} \mathrm{30-min} \mathrm{washes} \mathrm{were} \mathrm{conducted} \mathrm{in} \mathrm{sequence} \mathrm{with} \mathrm{the}$ aqueous solution until the concentration of drug in the washes reached a plateau. ${ }^{73}$

Stout et al. compared the methods reported by Cairnes et al. and Baumgartner and Hill to determine which was most effective at removing $\mathrm{COC}$ from the surface of hair that was intentionally contaminated and then exposed to a synthetic sweat solution. Neither method was found to be successful for complete removal of drug applied to the hair, a result consistent with a number of other studies aiming to remove COC external contamination. ${ }^{66,69,72,75,109,112}$ These authors also studied the effects of different decontamination methods on hair morphology by means of scanning electron microscopy, finding that each method could be associated with some level of damage to hair structures. ${ }^{70}$ Observed damage included lifting of the cuticle cells, changes to the cell membrane complex between cuticle cells, and substantial degradation of the cuticle, which in some cases resulted in complete loss of cuticle cells. Finally, Cuypers et al. reported that the use of certain solvents for the purpose of decontamination may in fact encourage the migration of the drug into deeper hair structures, questioning the currently held approaches for decontamination procedures. ${ }^{66}$ 
While much investigation into the decontamination of $\mathrm{COC}$ from hair has been conducted, there are fewer reports examining other common drugs of abuse. Tsanaclis et al. applied the use of ratios of drug present in wash solutions and extracts to the decontamination of THC, finding that two washes of less than 1 min each with methanol were sufficient. ${ }^{105}$ Duvivier et al. tested a series of washes for the removal of THC from the surface of hair, finding that the most effective methods were multi-step processes with methanol and SDS. ${ }^{76}$ Mantineiks et al. studied the decontamination of hair intentionally contaminated with methamphetamine, finding that, compared to heptane, DCM, acetonitrile, and 2-propanol, methanol was the most effective organic decontamination solvent for this compound. ${ }^{75}$ The results of the present work support the data reported by Baumgartner and Hill, in that a minimum of four washes is necessary to sufficiently decontaminate the surface of hair. Though the specific parameters under which the maximum amount of drug was removed from the surface of hair was analyte specific, as noted in each of the previously mentioned works.

The reported applications of DoE to optimizing conditions for forensic hair analysis are currently quite limited. Mueller et al. compared a number of pre-treatment parameters in the analysis of ethyl glucuronide in hair using a Plackett-Burman experimental design. ${ }^{110}$ However, the Plackett-Burman design does not consider combinatorial effects of the factors under study on the dependent variable. Alladio et al. used a 2-factor full factorial design to investigate and optimize the extraction of ethyl glucuronide from hair. ${ }^{50}$

An important result of the present study, as compared with previously reported decontamination results, is that only a limited number of treatments for each drug were found to be most effective at decontaminating the surface of hair. For example, effective 
decontamination of HER was associated with only a small number of design points, with much poorer results demonstrated for the other combinations. Conclusions about the most effective decontamination protocols for each analyte can be drawn from the commonalities between the treatment groups (i.e., design points), as summarized for the present study in Table 19. One might be tempted to rationalize these optimal conditions using drug solubility, polarity, ionization parameters, and the penetrability of the hair sample, all of

which have been hypothesized to influence the efficacy of wash parameters. ${ }^{75}$ However, it must be noted that the mechanisms of surface binding of drugs to hair components are poorly understood, making predictions about optimal wash conditions difficult at best. Furthermore, the major advantage of the DoE approach as applied herein is to elucidate combinations of parameters that would result in effective removal of surface contamination, a goal that would be challenging to accomplish a priori based on consideration of physicochemical characteristics alone.

\subsection{Conclusions}

The present study demonstrated proof of principle for the successful use of a DoE approach to identify effective combinations of experimental parameters for hair decontamination for a physically and chemically diverse group of drugs. The use of a $2^{4}$ fractional factorial block design for this purpose is a novel approach and provides additional insight into the effect of all considered wash parameters simultaneously, which has not been reported to date. Each of the analytes under study was found, within a limited set, to have a unique set of decontamination conditions that were most effective. Additional studies are necessary to test the applicability of the DoE approach and the optimized decontamination methods identified in the present study with authentic hair specimens. 


\section{SYSTEMATIC COMPARISON OF EXTRACTION PARAMETERS IN FORENSIC}

\section{HAIR ANALYSIS VIA $2^{3}$ FULL FACTORIAL DESIGN}

\subsection{Introduction}

In addition to the specific attention given to the decontamination protocol as described above, it is important to compare the remaining pre-treatment parameters, particularly those involved in the extraction of incorporated drug from hair. The most commonly used methods for the extraction of drug from hair include acidic, alkaline, or enzymatic degradation of the proteinaceous matrix or solvent swelling to release drug by passive diffusion, much like the technique used to incorporate/entrap drugs to generate HRM. ${ }^{39,47,51}$ Within these techniques, drug can be extracted for different amounts of time, at different temperatures, and with varying ratios of extraction agent to the mass of hair from which analytes are to be isolated.

Much of the published literature that exists comparing different techniques that have been successfully employed for the pre-treatment of hair samples and their subsequent analysis are review articles that tabulate results of these different approaches without comparing their performance. ${ }^{6,39,41,43,45,114-116}$ In contrast, Wada et al. used a different approach to reviewing existing methods by comparing established techniques by the class of drug, using the LOD/LOQ to determine the efficacy of these methods. ${ }^{44}$ Previous work aiming to more specifically study pre-treatment parameters has been primarily focused on individual factors. For example, Salomone et al. determined that pulverizing hair into a powder before determining the ethyl glucuronide $(\mathrm{EtG})$ content was more effective than cutting the hair into snippets. ${ }^{49}$ Studies conducted by Romano et al. and Stout et al. sought to compare whole decontamination protocols that were previously established for removal 
of COC, both finding that different decontamination protocols resulted in different amounts of drug removed from hair. They also determined that these protocols were insufficient in removing all of the contamination and, in the worst cases, actually encouraged incorporation of contaminating drug into the hair. ${ }^{69,74}$

Broecker et al. studied the effect of heat and ultrasonication on the efficacy of the solvent extraction technique commonly used in hair testing, finding that, while ultrasonication did not improve extraction of 17 drugs and metabolites from hair, maintaining a temperature of $37^{\circ} \mathrm{C}$ throughout the extraction did..$^{51}$ Duvivier et al. studied a variety of aqueous and organic decontamination solvents as single washes and in different combinations for the removal of $\Delta^{9}$-THC from the surface of hair, finding that there were several adequate sequences of washes that were equally effective. ${ }^{76}$ Madry et al. studied the efficacy of different extraction solvents for a wide variety of drugs and their metabolites, concluding that acidified methanol was most effective for the largest number of compounds in the study. ${ }^{117}$ However, none of these works directly compared all of the parameters that must be considered when deciding the pre-treatment parameters to apply.

A few recent studies have applied DoE techniques to hair analysis parameters on a limited basis. For example, Mueller et al. studied whole extraction protocols for isolating ethyl glucuronide (EtG), a metabolite routinely analyzed in hair as a marker for ethanol use and abuse, from hair. ${ }^{110}$ They compared several pre-treatment parameters, including extraction solvent, the use of ultrasonication, incubation time, incubation temperature, solvent amount, and hair particle size using a Plackett-Burman experimental design. ${ }^{110}$ This design assumes that the 2-factor effects (i.e., interactive effects coming from 2 factors as opposed to a single factor) are negligible. The factors identified to have significant 
influence on the detection of EtG included type of extraction solvent, extraction temperature, and particle size of the homogenized hair. ${ }^{110}$ Another study by Alladio, et al. supported these results and investigated their own optimized and validated methodology. First, a 2-factor full factorial design was used to investigate extraction temperature and solvent formulation. The optimization design was a face-centered central composite design comparing extraction time, temperature, and $\mathrm{pH}^{50}$ While useful, these Plackett-Burman experimental designs fail to address combinatorial effects. In the present study, the DoE approach was a $2^{3}$ full factorial design, as employed in the present work, allowed for the observation of both the individual and combinatorial effects of the parameters under study for a wider range of parameters than previously reported as there were three factors under study: extraction time, extraction temperature, and extraction agent to mass of hair ratio. Consequently, a $2^{3}$ design was employed (Table 20) under the hypothesis that DoE could be used to determine which extraction parameters were best for each analyte and illustrate similarities between extraction parameters that may be adequate for all analytes under study.

Table 20: $2^{3}$ Full Factorial Design for the Comparison of Extraction Parameters

\begin{tabular}{|c|c|c|c|c|c|c|c|}
\hline Design Point & A & B & C & AB & AC & BC & ABC \\
\hline$(1)$ & - & - & - & + & + & + & - \\
\hline $\mathrm{a}$ & + & - & - & - & - & + & + \\
\hline $\mathrm{b}$ & - & + & - & - & + & - & + \\
\hline $\mathrm{c}$ & - & - & + & + & - & - & + \\
\hline $\mathrm{ab}$ & + & + & - & + & - & - & - \\
\hline $\mathrm{ac}$ & + & - & + & - & + & - & - \\
\hline $\mathrm{bc}$ & - & + & + & - & - & + & - \\
\hline $\mathrm{abc}$ & + & + & + & + & + & + & + \\
\hline
\end{tabular}


5.2 Materials and Methods

\subsubsection{Chemicals and Solvents}

Solvents and chemicals used for the preparation of mobile phases and sample preparation for LC-MS analysis (water, methanol, 2-propanol, dichloromethane, formic acid, ammonium formate) were of HPLC-grade and obtained from Fisher Scientific (Hampton, NH, USA). Stock solutions of AMP, AMP-D11 DZP, HER, COC, DZP-D5, MOR-D3, and COC-D3 were obtained at $1.0 \mathrm{mg} / \mathrm{mL}$ in methanol from Cayman Chemical (Ann Arbor, MI, USA). $\Delta^{9}$-THC and $\Delta^{9}$-THC-D3 were purchased from Cayman Chemical as 10.0 and $1.0 \mathrm{mg} / \mathrm{mL}$ solutions in acetonitrile, respectively. These stock solutions were used in the preparation of the wet chemistry-based incorporated HRM and calibration curves. DTT was purchased from Sigma-Aldrich (St. Louis, MO, USA), and Proteinase-K was obtained from Invitrogen (Carlsbad, CA, USA). Three kDa molecular weight cut off PTFE spin filters were obtained from MilliporeSigma (Burlington, MA, USA). Single donor, unprocessed, natural black color human hair $(\sim 300 \mathrm{~g})$ was obtained from a commercial source.

\subsection{2 $2^{3}$ Design Matrix for Comparison of Extraction Technique}

In general, and depicted in Figure 10 the DoE samples were prepared by first weighing $30 \mathrm{mg}$ of the incorporated HRM into a $20 \mathrm{~mL}$ amber vial. The aqueous wash was performed by adding $1 \mathrm{~mL}$ aqueous decontamination solvent and agitating on an orbital shaker for 1 min. Next, the organic wash was performed with $1 \mathrm{~mL}$ of solvent and agitation on an orbital shaker for $1 \mathrm{~min}$. The decontamination conditions applied were those that were established to be most effective in the previous chapter. The hair was then dried for $24 \mathrm{~h}$ at $37^{\circ} \mathrm{C}$ in a 
temperature-controlled oven. Once dried, the samples were either cut into snippets or powdered. Samples that were to be snippets were cut with surgical scissors into $\sim 1 \mathrm{~mm}$ segments. Samples that were powdered were milled with 10 chrome-steel milling beads in chrome-steel milling tubes using a Mini-BeadBeater 24 ball mill (Biospec; Bartlesville, OK, USA) at $3200 \mathrm{rpm}$ for $30 \mathrm{~s}$. Drug was then extracted using one of the two previously described techniques, enzymatic degradation or solvent swelling of the hair matrix, with a volume of extraction agent.

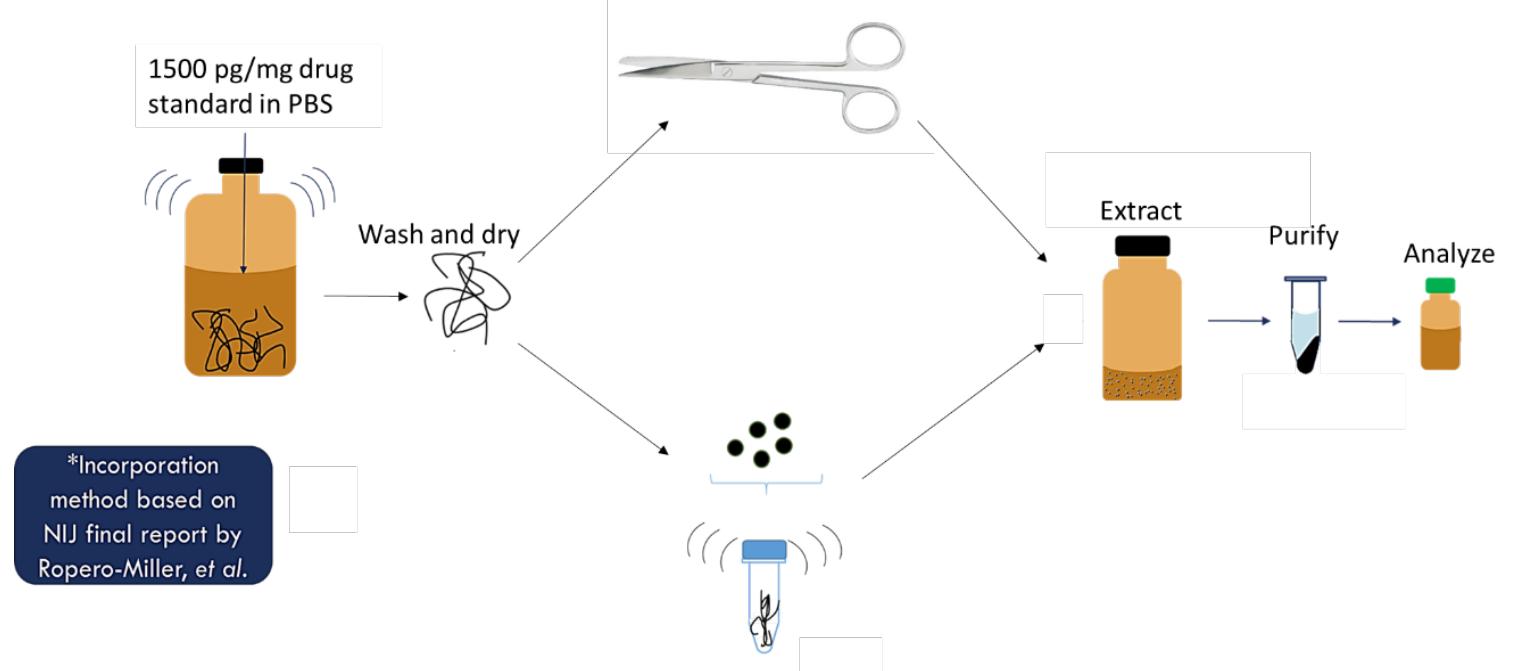

Figure 10: Schematic of sample preparation for extraction DoE studies.

Table 21 defines each factor and each level of these factors that was studied in the DoE designs, and Table 20 outlines the $2^{3}$ full fractional design used to study these parameters. Each design point in Table 20 correspond to a single sample to which different levels of these parameters were applied. Extracted samples were purified by centrifugation and SPE before LC -MS analysis, as described in the previous chapter. A randomized list of design points was used to determine the order in which samples were prepared and analyzed. Three replicate samples for each design point as listed in Table 20 were analyzed. One 
replicate study of the entire DoE protocol was conducted for each of the two extraction techniques.

Table 21: Definition of factors and factor levels for each experimental design.

\begin{tabular}{|c|c|c|}
\hline Factor & \multicolumn{2}{|c|}{ Pre-treatment Design } \\
\cline { 3 - 3 } A & Extraction solvent volume ratio to sample size & $(+) 25 \mu \mathrm{L} / \mathrm{mg}$ \\
\cline { 3 - 3 } B & Particle size of homogenized hair & $(-) 12.5 \mu \mathrm{L} / \mathrm{mg}$ \\
\cline { 3 - 3 } C & Extraction Time & $(-)$ powder \\
\cline { 3 - 3 } & & $(+) 24 \mathrm{~h}$ \\
\hline
\end{tabular}

\subsubsection{Statistical Analysis}

LC-MS analysis allowed for the quantification of drug from all of the samples analyzed. The replicate samples containing drug isolated using each extraction technique were compared by means of a Student's t-test to determine which, if either, of the techniques resulted in more efficient isolation of the analyte from the matrix.

The quantity of drug detected in the triplicate extracts, from the technique with the highest recovery, was then used to find the statistical parameters necessary to complete an ANOVA table comparing the parameters under study in the DoE matrix. If a significant difference was observed between treatment groups (i.e., design points) by means of the ANOVA F-test, Tukey's HSD post-hoc analysis was conducted to determine which parameters or combinations of parameters resulted in this difference. JMP software $\left(13^{\text {th }}\right.$ ed.) was used for all statistical analyses. 


\subsection{Results}

\subsubsection{Comparison of Extraction Technique}

A pooled Student's t-test indicated which of the two extraction techniques resulted in the highest extraction of each analyte, as seen in Figure 11. The enzymatic degradation technique was significantly $(\alpha=0.05)$ more effective at extracting AMP $(p=0.01)$ and the solvent swelling technique was most effective at extracting DZP $(\mathrm{p}=0.001)$. There was no significant difference in the extraction of THC between the two techniques $(p=0.7)$, though the mean of the samples extracted by the solvent technique was slightly higher than that of the enzymatic degradation technique; thus the solvent technique was used in the subsequent experiments for DZP and THC and the enzymatic degradation technique was used for AMP. 

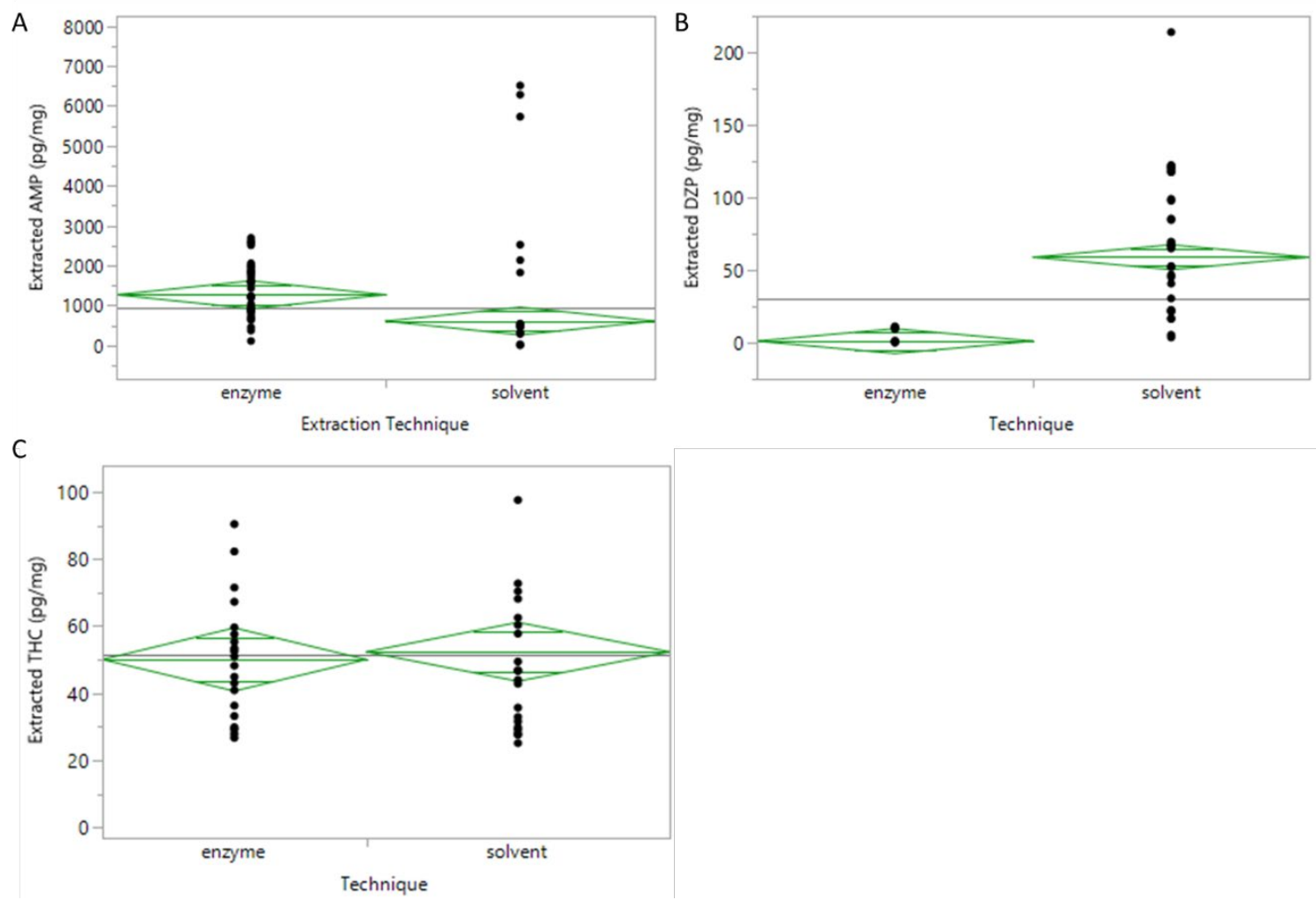

Figure 11: Comparison of extracted drug with different extraction techniques. Points represent each sample extracted using the designated technique $(n=48)$, with different pretreatment protocols as outlined in the DoE matrix. The outlines in green represent the quantiles of the data. Solid, central, horizontal line represents the average extracted quantity for each technique for AMP (A), DZP (B), and THC (C).

\subsubsection{DoE Comparison of Extraction Conditions}

ANOVA F-Test indicated that all factors and their combinations were significant, with p-values less than 0.05 (i.e., the $\alpha$-level). Each analyte under study had different conditions under which extraction was the highest. Extraction conditions associated with design point c resulted in the highest extracted quantity of AMP, at 4,316 \pm 96 pg (Figure 12A). The specific conditions applied to the samples in the treatment group associated with design point c were extracting drug from hair that was pulverized into a powder before being exposed to $12.5 \mu \mathrm{L}$ of extraction solvent/mg of hair for $24 \mathrm{~h}$. The conditions under which 
the most DZP, at $122 \pm 1 \mathrm{pg}$ (Figure 12B), was isolated from the incorporated HRM were associated with design point $b$; this means that DZP was extracted from samples in this treatment group by cutting the hair into $1 \mathrm{~mm}$ snippets and applying $12.5 \mu \mathrm{L}$ of extraction solvent/mg of hair for $2 \mathrm{~h}$. The greatest amount of THC extracted, at $115 \pm 1 \mathrm{pg}$, was observed for samples in the treatment group corresponding to design point ab (Figure 12C). THC was extracted from samples in this group by cutting the hair into $1 \mathrm{~mm}$ snippets and exposing them to $25 \mu \mathrm{L}$ of extraction solvent/mg of hair for $2 \mathrm{~h}$. 
A

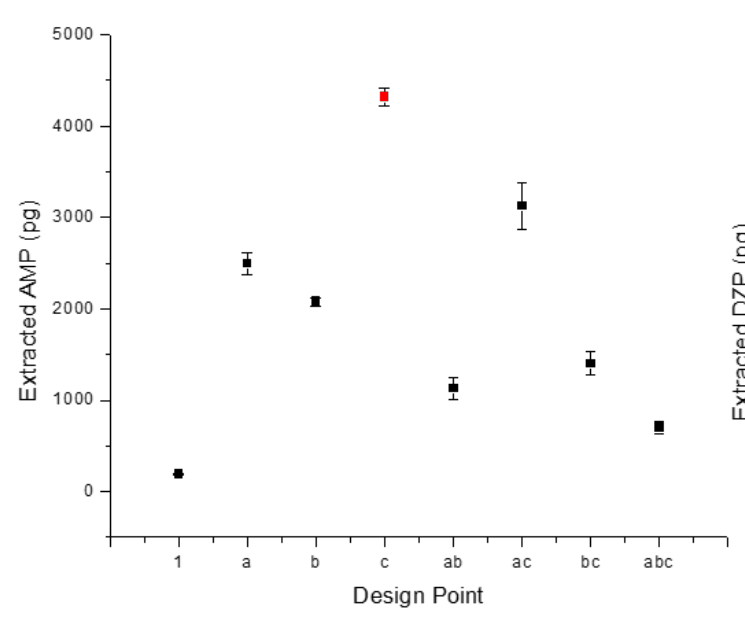

C
B

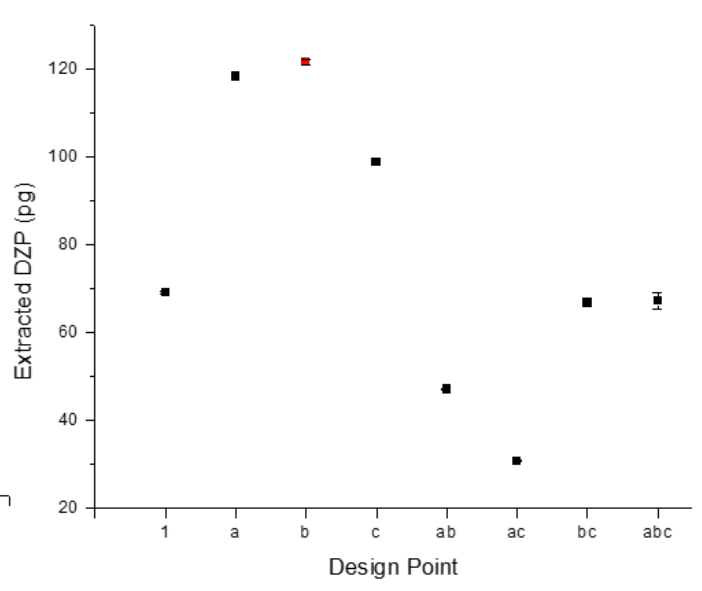

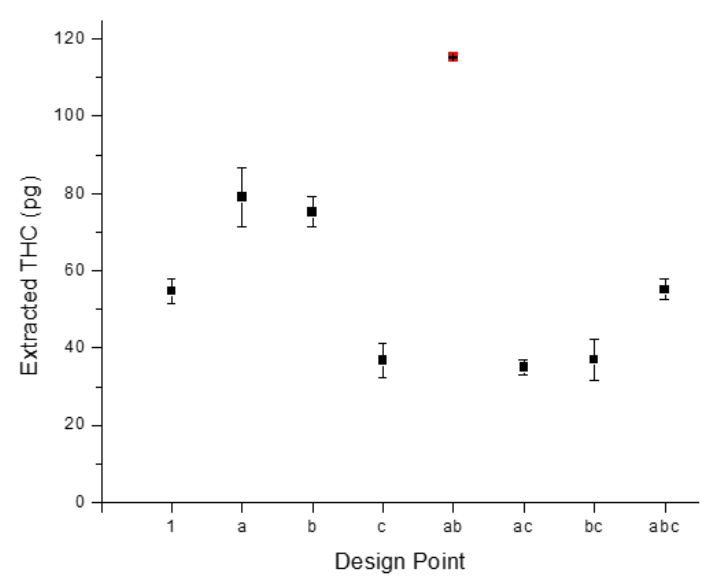

Figure 12: Comparing the effect of the variation in extraction parameters on the amount of extracted drug for AMP (A), DZP (B), and THC (C).

\subsection{Discussion}

All factors and combinations of factors in the DoE studies of hair pre-treatment conditions were found to be significant, suggesting that the factors alone did have a significant effect on the level of drug detected in each sample, highlighting the importance of employing a factorial design to investigate all combinations of parameters, as was noted by Alladio et al. ${ }^{50}$ Moreover, the higher-level effects, representing combinations of the 
main effects, also had significant impact on extracted drug quantities, which cannot be observed using other designs like the Plackett-Burmann design used by Mueller et al. ${ }^{110}$ This means that it is essential to study pre-treatment conditions as whole protocols, as the interaction between all of the factors under consideration have a significant effect on the efficacy of the extraction of drug from the matrix.

AMP was most effectively extracted from the matrix by minimizing the hair particle size by powdering the hair. This is consistent with previous literature, ${ }^{49}$ and is likely because drugs that are incorporated into hair are hypothesized to be localized in the cortex of the hair. ${ }^{47,96}$ When the hair strand is pulverized, more of the cortex is exposed to the extraction solution than when the hair is cut into $1 \mathrm{~mm}$ segments. Previous studies have also shown the significance of particle size on recovery of ethyl glucuronide from hair, indicating that a smaller sample size results in more efficient extraction of drug. ${ }^{49,76}$ However, extraction of DZP and THC from hair was maximized by cutting hair into snippets rather than pulverization; this may be explained by the fact that at a $\mathrm{pH}$ of 7.4, as was the case in the present work, both DZP and THC are neutral while AMP is cationic. The charged AMP may be held in the matrix by different mechanisms that the DZP and THC at this $\mathrm{pH}$, which could impact its ability to be released from the matrix, resulting in different recovery of drug. This technique has also been used successfully for a variety of analytes. ${ }^{39}$ The maximization of AMP and DZP extraction was achieved with $12.5 \mu \mathrm{L}$ of extraction solvent/mg of hair, where THC required $25 \mu \mathrm{L}$ of extraction solvent $/ \mathrm{mg}$ of hair. Previous studies on ethyl glucuronide indicated that the optimal conditions for extraction were at $25 \mu \mathrm{L}$ of extraction solvent/mg of hair. ${ }^{50,110} \mathrm{EtG}$ has a TPSA of $116 \AA^{2}$ and a pKa of 3.5; thus at $\mathrm{pH} 7.4$ this very polar molecule is neutral. The analytes in the present work 
were much less polar than EtG, which may help to explain the differences in these results. These same studies indicated that extraction for $24 \mathrm{~h}$ was optimal, which is consistent with the results for AMP. ${ }^{50,110}$ However, DZP and THC were most effectively extracted after 2 $\mathrm{h}$, which may again be related to the difference in the charge state of these molecules at physiological $\mathrm{pH}$.

\subsection{Conclusions}

The highest extraction of AMP, DZP, and THC involved the use of the solvent swelling technique to isolate drug from hair. This is likely because it is a gentle technique, to minimize the probability of chemically modifying the analytes, that relies on simple diffusion for the release of drug from hair. Although one of the aims of this work was to find similarities in pre-treatment conditions between different analytes, results indicate that each analyte must be considered individually, as the conditions under which the most drug will be extracted from hair varies. Neutral compounds were more effectively isolated from the matrix with shorter extraction times and larger particle sized of the homogenized hair, while the cationic AMP required smaller particle size and longer extraction for effective extraction. 


\section{PROBING INTERACTIONS BETWEEN DRUGS OF ABUSE AND EUMELANIN}

\subsection{Introduction}

It is clear that drugs of abuse can become trapped within human head hair $^{4,11,98,99,13,15,20,45,54,55,64,96}$, which necessitates the question: how do these substances remain held within the hair? It has been demonstrated that drugs can associate with

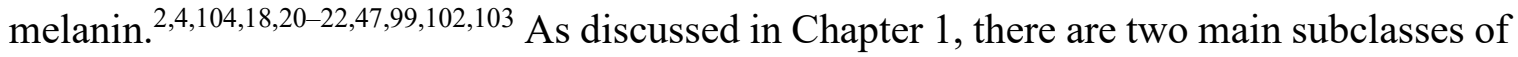
melanins: eumelanin and pheomelanin. Eumelanin is the pigment primarily associated with brown/black hair coloration; much of the research regarding the association of drugs of abuse with matrix components have referenced the ability of drugs to interact with eumelanin. ${ }^{4,15,16,20,118-121}$ Different amounts of the subclasses of melanin are present within the hair of people from different ethnic and racial backgrounds; these inter-individual differences in pigmentation may lead to complications in the interpretation of toxicological analyses using hair. ${ }^{5}$

While it has been demonstrated that eumelanin and drugs can interact with one another as a mechanism by which drugs may be retained in hair, the nature of these associations is not well understood. It has been suggested that basic, lipophilic drugs may have a greater affinity for binding/interacting with melanin. ${ }^{47}$ The preferential binding of basic drugs would be favored as a result of anionic carboxylic acids on the structure of melanin, indicated in Figure 13, which at the natural $\mathrm{pH}$ of hair (i.e., 4.5 - 5.5), up to half of the carboxylic acids in melanin would be charged. ${ }^{13}$ This preferential binding is supported by other data that show the binding preference between compounds with an ionizable primary amine, like amphetamine, and eumelanin. ${ }^{4,15,20,122}$ It has also been suggested that peptide 
bonds can form between amine groups and these carboxylic acid functions, especially those associated with eumelanin. ${ }^{15,20}$

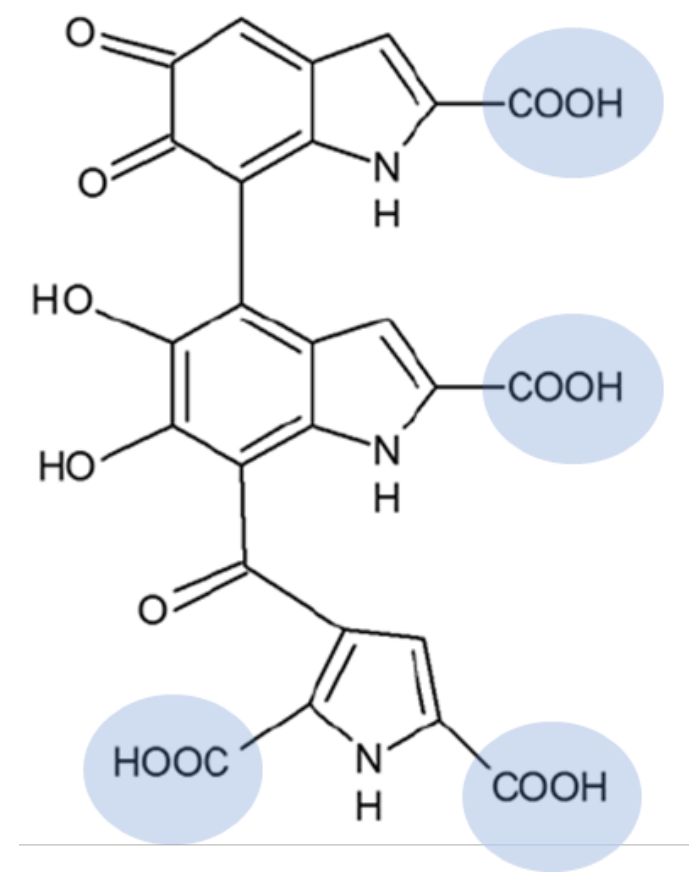

Figure 13: Monomeric unit of eumelanin. ${ }^{21}$ The carboxylic acid moieties (highlighted in blue) have a $\mathrm{pK}_{\mathrm{a}} \sim 5.0$, and have been hypothesized to interact with cationic drug functionalities under neutral and alkaline conditions (i.e., $\mathrm{pH} \geq 7$ ), in which these carboxylic acids would be deprotonated, and therefore anionic.

A study by Cappelle et al. compared the detection of drugs of abuse in hair and nails, both keratinous matrices formed from type I and type II IFs. ${ }^{123}$ The notable differences in the matrices were the mechanisms by which drugs could become incorporated into the matrix and the presence or absence of melanin. ${ }^{123}$ Drugs can become incorporated into hair by means of drug-containing blood flow to the follicle, while drugs can become incorporated into nails by means of blood flow to the nail matrix and the nail bed, resulting in vertical and horizontal incorporation of drug into the nail. Hair contains melanin, whereas nails do not; some substances in hair have shown a positive relationship with 
melanin content. ${ }^{3-5,7,20,25,47,123}$ The substances most noted to associate with eumelanin have been basic compounds. However, it is still possible to detect drugs of abuse in melaninfree nail matrices. Therefore, it is clear that drugs of abuse can associate with the keratinous matrix and/or melanin, when it is present.

Several analytical techniques have been employed to investigate the associations between xenobiotics and melanin including NMR, liquid scintillation counting, UV/Vis, and MS. Salazar-Bookaman et al. studied the interaction of drugs with a synthetic, water soluble eumelanin-related compound, (-)-dopa melanin, via line width measurements acquired using a pulse NMR instrument. The spectra of dilute melanin mixed with ophthalmologic pharmaceuticals (i.e., atropine, acetylcholine isoprenaline bitatrate, ephedrine, and timolol meleate) demonstrated line broadening of the peaks associated with the $\mathrm{N}$-alkyl protons on the respective structures as compared to the spectra of these compounds in the absence of melanin. ${ }^{124}$ A specific example from this work, is given for ephedrine (Figure 14).

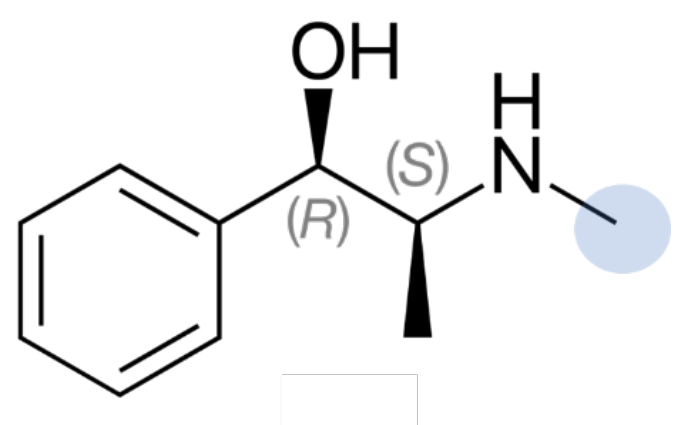

Figure 14:Structure of ephedrine. The protons of interest for the study of the dissociation constant with melanin were the $N$-methyl protons implicit in the blue highlighted area. 
The line broadening of the N-methyl protons at $2.57 \mathrm{ppm}$, as noted in Figure 15, were recorded as drug was titrated into a dilute solution of the water-soluble melanin to then calculate the dissociation constant.

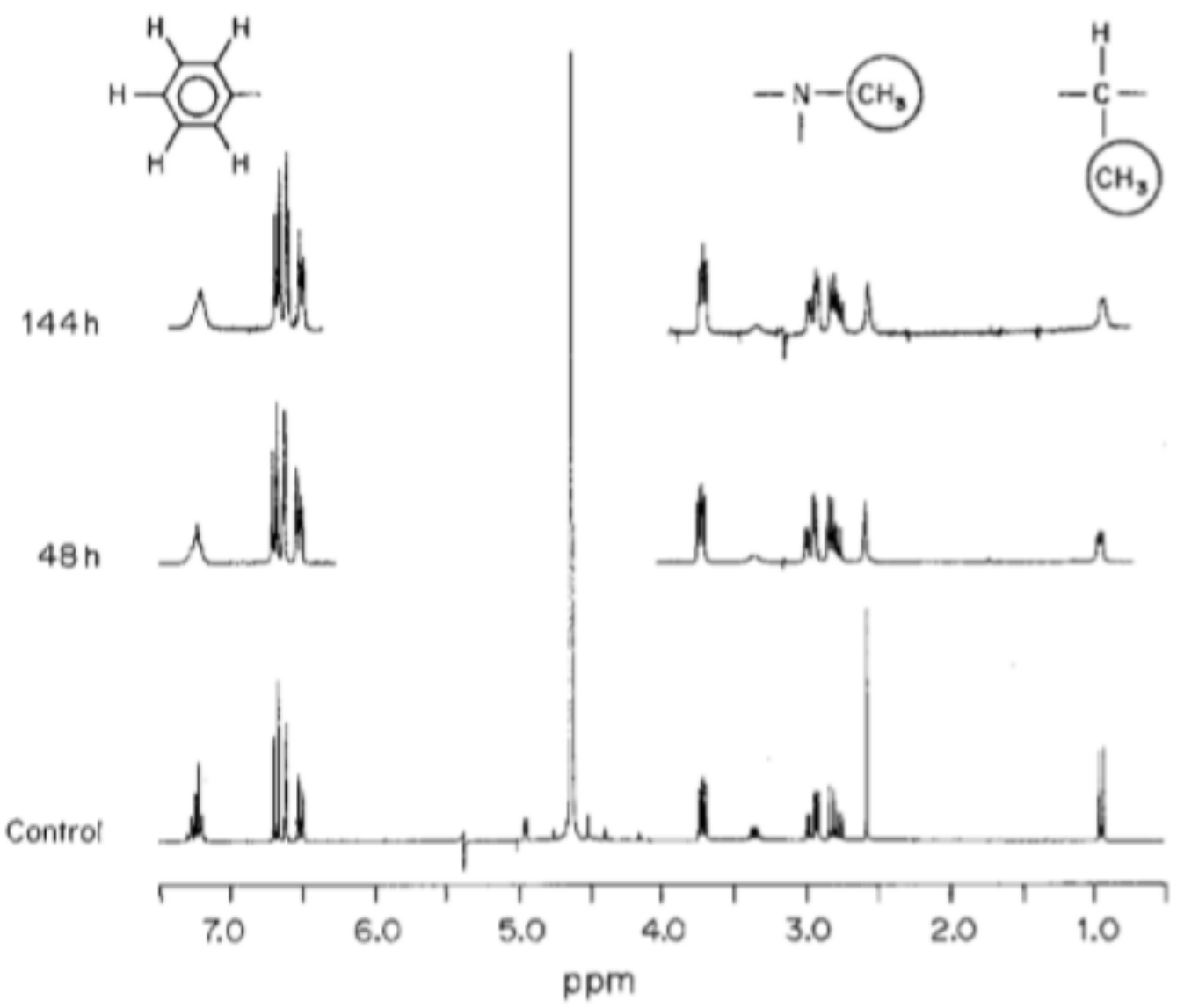

Figure 15: Spectra of ephedrine in the absence of melanin (bottom spectrum) and in the presence of melanin (top two spectra). The assignments of some spectral peaks are noted at the top of the figure. The $N$-methyl peaks, at $2.57 \mathrm{ppm}$, were noted to broaden in the presence of melanin and remain broad after $48 \mathrm{~h}$ and $144 \mathrm{~h} .{ }^{124}$

The upward curve of the fitted trendline, as seen in Figure 16, indicated that there was more than one binding site on melanin that ephedrine interacted with, with dissociation constants for these sites reported as $\mathrm{K}_{\mathrm{d} 1}=2.08 \mathrm{mM}$ and $\mathrm{K}_{\mathrm{d} 2}>20 \mathrm{mM}{ }^{124}$ 


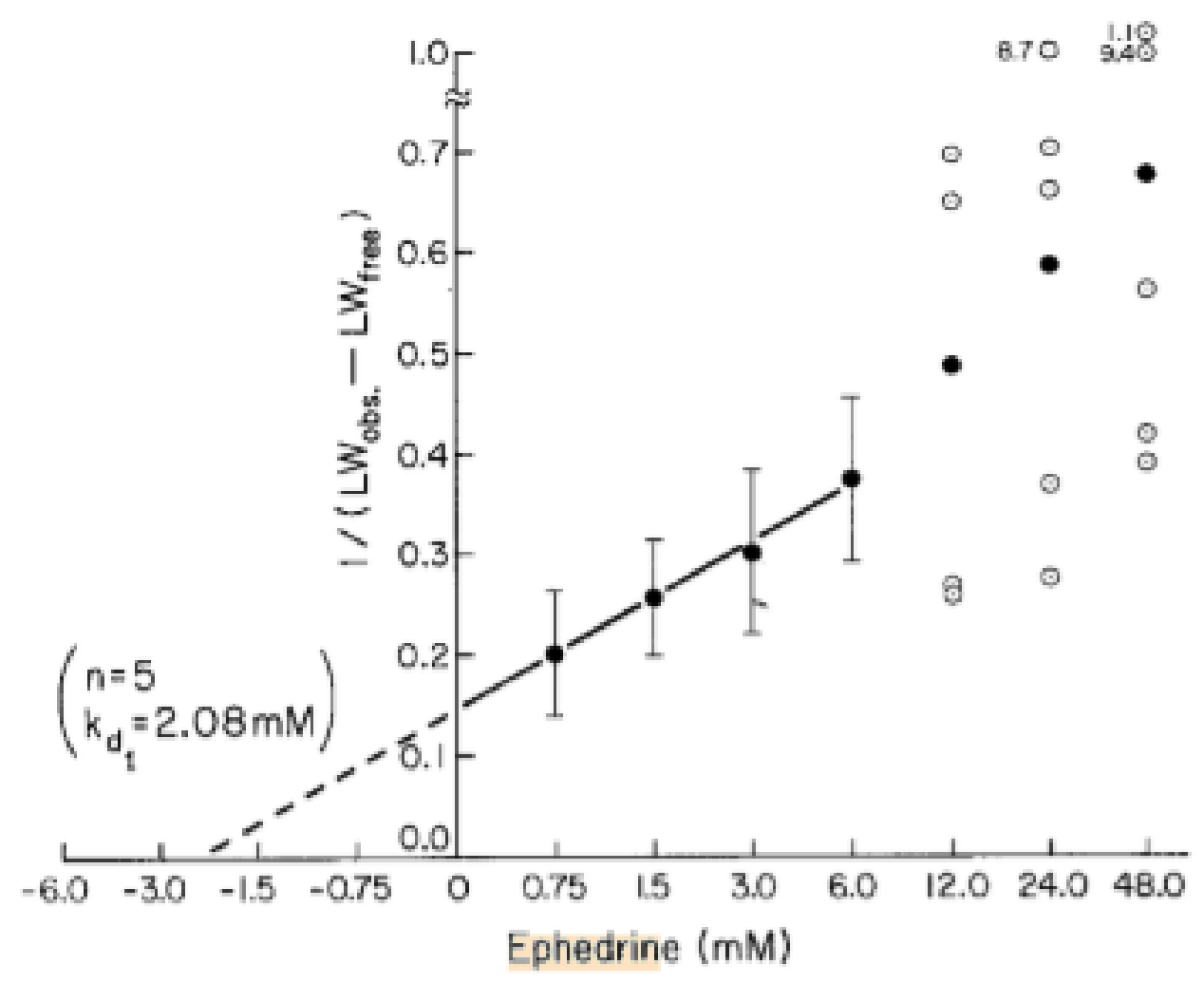

Figure 16: Fitted trendline for the change in the line width of 2.57 ppm peak of ephedrine titrated into melanin. ${ }^{124}$

Another approach to studying these interactions is through the use of radiolabeled drugs and liquid scintillation counting. Shimada et al. studied the association between radiolabeled drugs in a PBS buffer at $\mathrm{pH} 7.4$ and eumelanin by titrating the melanin into the solutions of drug and quantitating the unbound drug. The free drug concentration was plotted against that of the bound drug and fit to a linearized Langmuir isotherm model, as shown in Figure 17. ${ }^{16}$ 

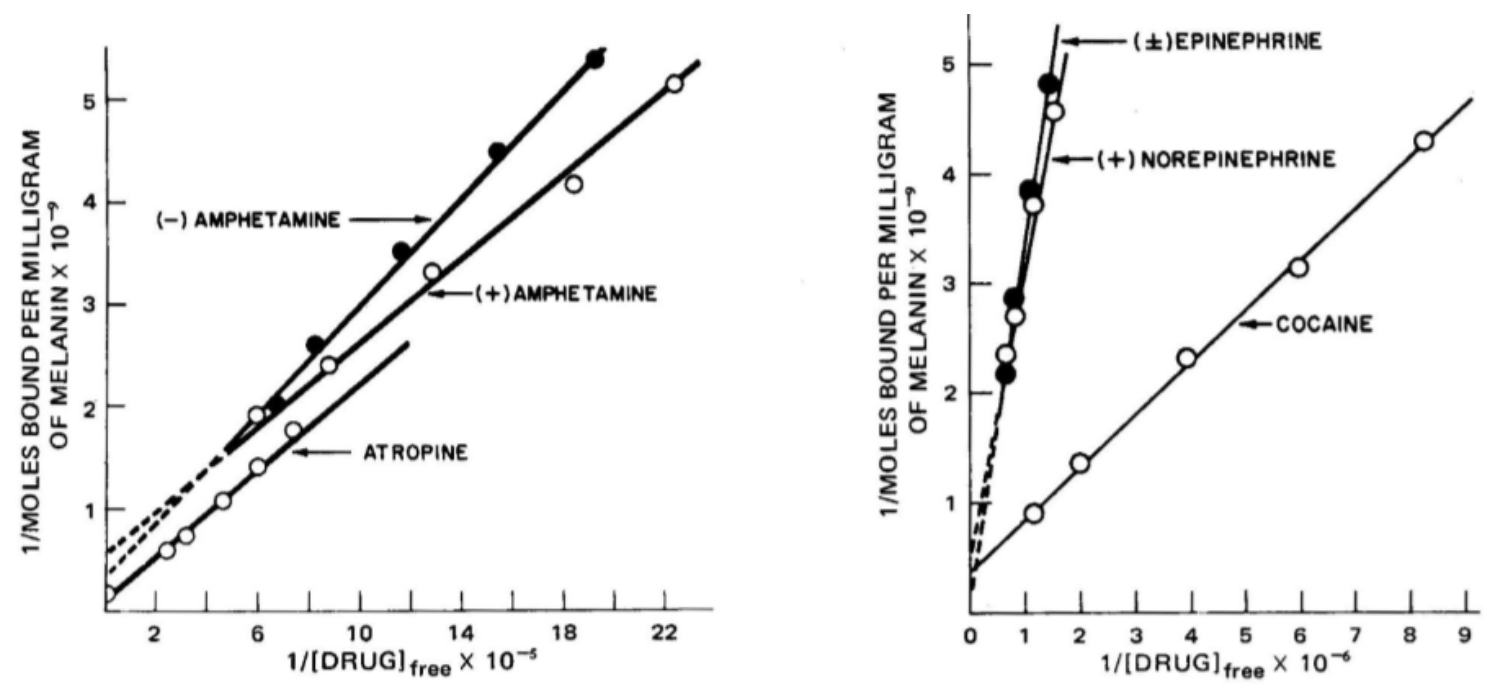

Figure 17: Linearized Langmuir isotherms of AMP, ephedrine, octopamine, COC, and norepinephrine. ${ }^{16}$

The binding constants for the drugs of abuse were calculated from the Langmuir fitted curves, as reported in Table 22. The highest melanin affinity was observed for COC (+) AMP, epinephrine and (-) AMP, at $8.9 \times 10^{5} M^{-1}, 2.8 \times 10^{5} M^{-1}, 2.6 \times 10^{5} M^{-1}$, and $1.1 \times 10^{5} M^{-1}$ respectively. $^{16}$ 
Table 22: Linearized Langmuir fit parameters and calculated melanin affinities for AMP ephedrine, octopamine, norepinephrine, atropine, and COC. ${ }^{16}$

\begin{tabular}{|c|c|c|c|c|}
\hline Drug & $\begin{array}{l}\text { Slope } \times 10^{-3} \\
(\text { Lower/Upper } \\
\text { Limit) }^{\mathrm{a}}\end{array}$ & $\begin{array}{c}\text { Intercept } \times 10^{-3} \\
(\text { Lower/Upper } \\
\text { Limit) }{ }^{\mathrm{a}}\end{array}$ & $\begin{array}{c}\text { Maximum } \\
\text { Moles Bound } \\
\text { per Milligram } \\
\text { of Melanin } \\
\end{array}$ & Affinity $^{\mathrm{c}}, \mathrm{M}^{-1}$ \\
\hline (-)-Amphetamine & $\begin{array}{c}2.7 \\
(2.5 / 2.9)\end{array}$ & $\begin{array}{c}3.1 \\
(-0.4 / 6.6)\end{array}$ & $3.2 \times 10^{-9}$ & $1.1 \times 10^{5}$ \\
\hline$(+)$-Amphetamine & $\begin{array}{c}2.1 \\
(1.7 / 2.4)\end{array}$ & $\begin{array}{c}5.7 \\
(1.0 / 10)\end{array}$ & $1.8 \times 10^{-9}$ & $2.8 \times 10^{5}$ \\
\hline ( \pm )-Epinephrine & $\begin{array}{c}3.5 \\
(3.1 / 3.9)\end{array}$ & $\begin{array}{c}0.25 \\
(-4.1 / 4.6)\end{array}$ & $39.7 \times 10^{-9}$ & $0.072 \times 10^{5}$ \\
\hline (-)-Ephedrine & $\begin{array}{c}4.0 \\
(3.3 / 4.8 \\
\end{array}$ & $\begin{array}{c}5.6 \\
(-0.6 / 11.7)\end{array}$ & $1.8 \times 10^{-9}$ & $1.4 \times 10^{5}$ \\
\hline (士)-Octopamine & $\begin{array}{c}2.8 \\
(2.2 / 3.3)\end{array}$ & $\begin{array}{c}7.2 \\
(0.8 / 14)\end{array}$ & $1.4 \times 10^{-9}$ & $2.6 \times 10^{5}$ \\
\hline$(+)$-Norepinephrine & $\begin{array}{c}2.7 \\
(2.4 / 3.1) \\
\end{array}$ & $\begin{array}{c}5.8 \\
(1.4 / 10) \\
\end{array}$ & $1.7 \times 10^{-9}$ & $2.1 \times 10^{5}$ \\
\hline Atropine & $\begin{array}{c}2.3 \\
(2.1 / 2.4) \\
\end{array}$ & $\begin{array}{c}0.57 \\
(0.07 / 1.1) \\
\end{array}$ & $17.6 \times 10^{-9}$ & $0.25 \times 10^{5}$ \\
\hline$( \pm)$-Cocaine & $\begin{array}{c}0.48 \\
(0.45 / 0.51)\end{array}$ & $\begin{array}{c}4.2 \\
(2.8 / 5.7)\end{array}$ & $2.4 \times 10^{-9}$ & $8.9 \times 10^{5}$ \\
\hline
\end{tabular}

${ }^{\mathrm{a}} 95 \%$ confidence intervals calculated at $\mathrm{p}=0.05 .{ }^{\mathrm{b}}$ Calculated as the reciprocal of the $y$ intercept. ${ }^{\mathrm{c}}$ Calculated from the intercept and slope.

Gautam et al. attempted to study the thermodynamics of the interaction of AMP and melanin by means of UV/Visible spectroscopy and Scatchard analysis. The effect of increasing concentrations of melanin on the spectrum of AMP resulted in a bathochromic shift, peak broadening, and an increase in absorbance at $208 \mathrm{~nm}$, as seen in Figure 18. These results were interpreted as representing the potential formation of peptide bonds between the primary amine of amphetamine and the carboxylic acid of melanin, as peptides exhibit $\lambda_{\max }$ values between 200 and $240 \mathrm{~nm}$ resulting from the $\mathrm{n} \rightarrow \pi^{*}$ transition of the carbonyl. Observing the changes of the spectrum in the region the authors attribute specifically to AMP, i.e., between 250 and $265 \mathrm{~nm}$, revealed a decrease in the absorbance with increasing amounts of melanin. The changes observed at $258.7 \mathrm{~nm}$ were used to perform Scatchard analysis. ${ }^{15}$ 


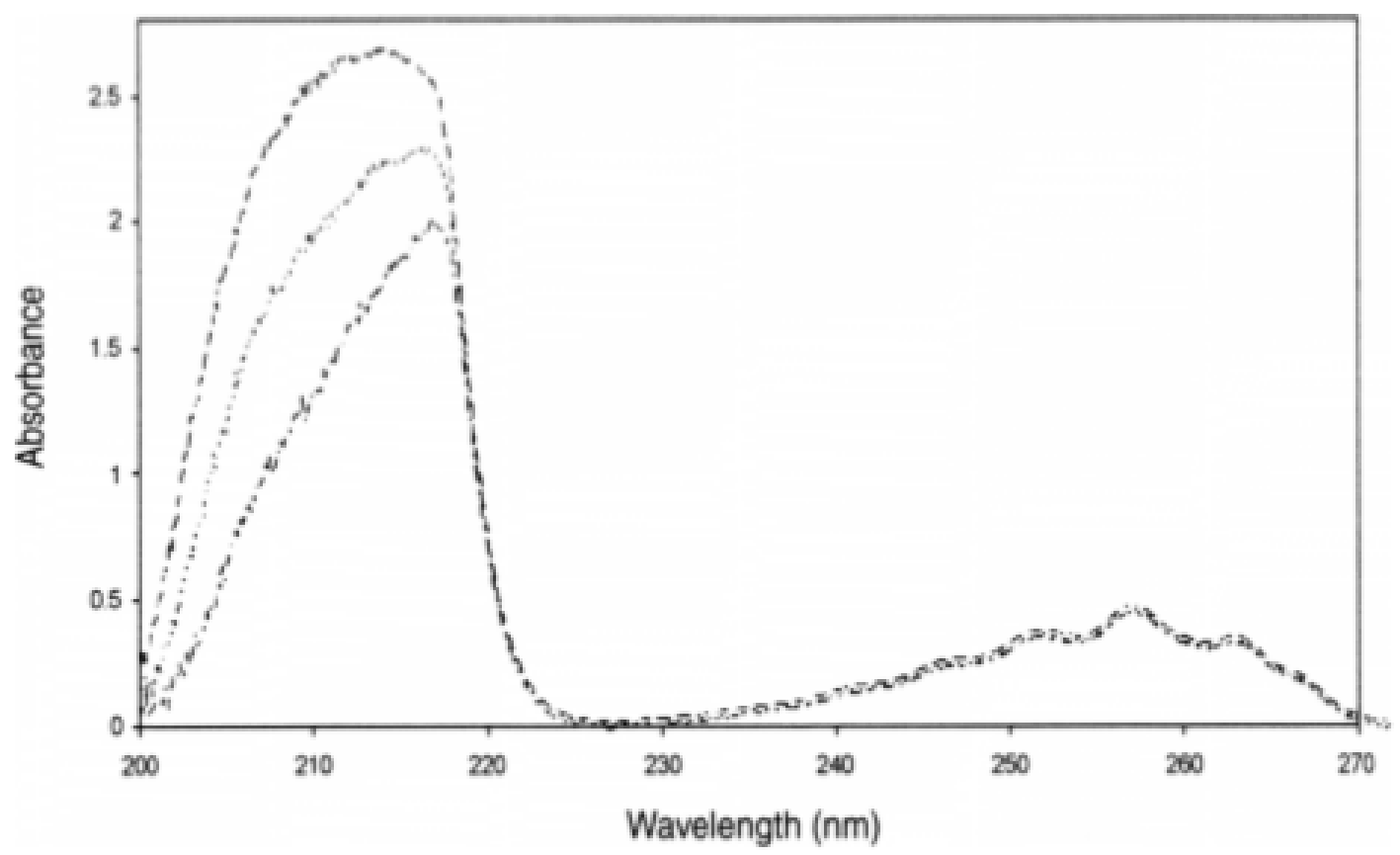

Figure 18: UV/Vis spectra of AMP with increasing concentrations of melanin. ${ }^{15}$

It was noted that the association between AMP and synthetic melanin reached equilibrium when $32 \%$ of the AMP in solution was bound to melanin. However, it was not possible to calculate the association constant via Scatchard analysis, which is a method to determine binding constants by conducting experiments in which increasing amount of a ligand are added to a solution of a substrate with which the ligand is known to interact such that saturation of the interaction site is eventually achieved. Since the exact structures of and thus the number of interaction/binding sites on melanin polymers are not known, it was impossible for the authors to determine the saturation point of melanin for the successful application of Scatchard analysis in this context. However, the curvilinear fit of the Scatchard plot indicated that there is more than one site at which AMP associates with melanin, supporting the Scatchard analysis results. ${ }^{15}$ 
Borges et al. used a similar radiolabeling method as Shimada et al. to study the binding of COC and AMP with eumelanin. Then, the experiments were repeated using LC-QqQ to quantify the unbound drug after purification by centrifugation. The bound fraction of drug was determined by mass balance. The data was then fit to a Langmuir isotherm for estimation of the $K_{a}$ and Scatchard analysis was performed to determine the number of binding sites. The Scatchard analysis reportedly indicated two types of binding sites on melanin: high affinity/low capacity sites and low affinity/ high capacity sites. The $\mathrm{K}_{\mathrm{a}}$ was calculated for each of these sites as reported in Table $23 .{ }^{4}$ The results obtained differed from those obtained by Shimada et al., which the authors attributed to the different buffers used in the two studies. Shimada et al. used a buffer with higher ionic strength, resulting in a lower $\mathrm{K}_{\mathrm{a}}$ value observed for the binding of AMP and $\mathrm{COC}$ to melanin as compared to that of Borges et al. ${ }^{4,16}$ Since the concentration of the ions in the buffer seemed to have an effect on the binding of drug to melanin, it is hypothesized that the present research will find that the physicochemical nature of the binding between drugs of abuse and eumelanin will be primarily ionic.

Table 23: Association constants between eumelanin and drugs as reported by Borges et $a l^{4}$

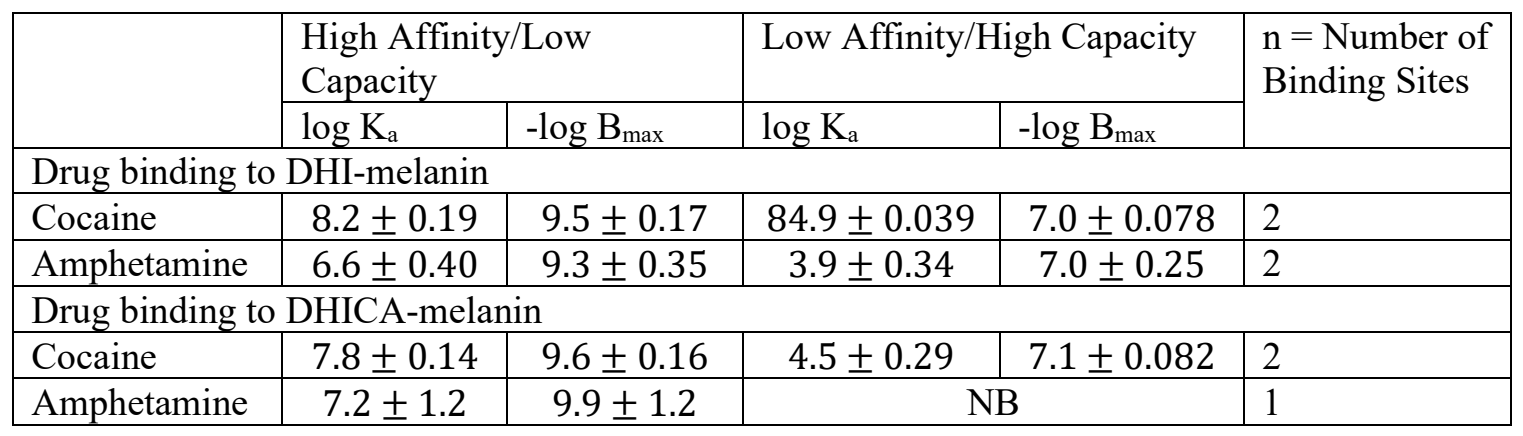


In addition to the binding studies, Borges et al. performed qualitative LC-MS/MS to investigate the potential for covalent melanin-drug adducts. Oxidized catechol, reduced catechol, deprotonated phthalic acid, and 3-methylindole were used as surrogates to represent functional groups of melanin with the potential for adduct formation with AMP and COC. The resultant spectra, seen in Figure 19, indicated that there may be non-covalent adducts formed between AMP and oxidized catechol through hydrogen bonding, as depicted int the upper-left corner of Figure 19C, suggesting that these adducts may also form with eumelanin. ${ }^{4}$ 


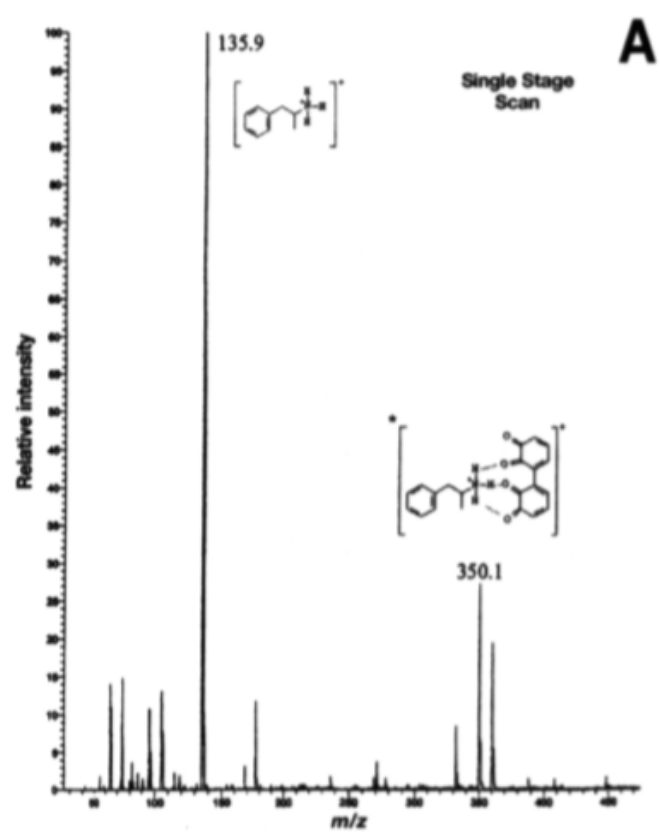

A B
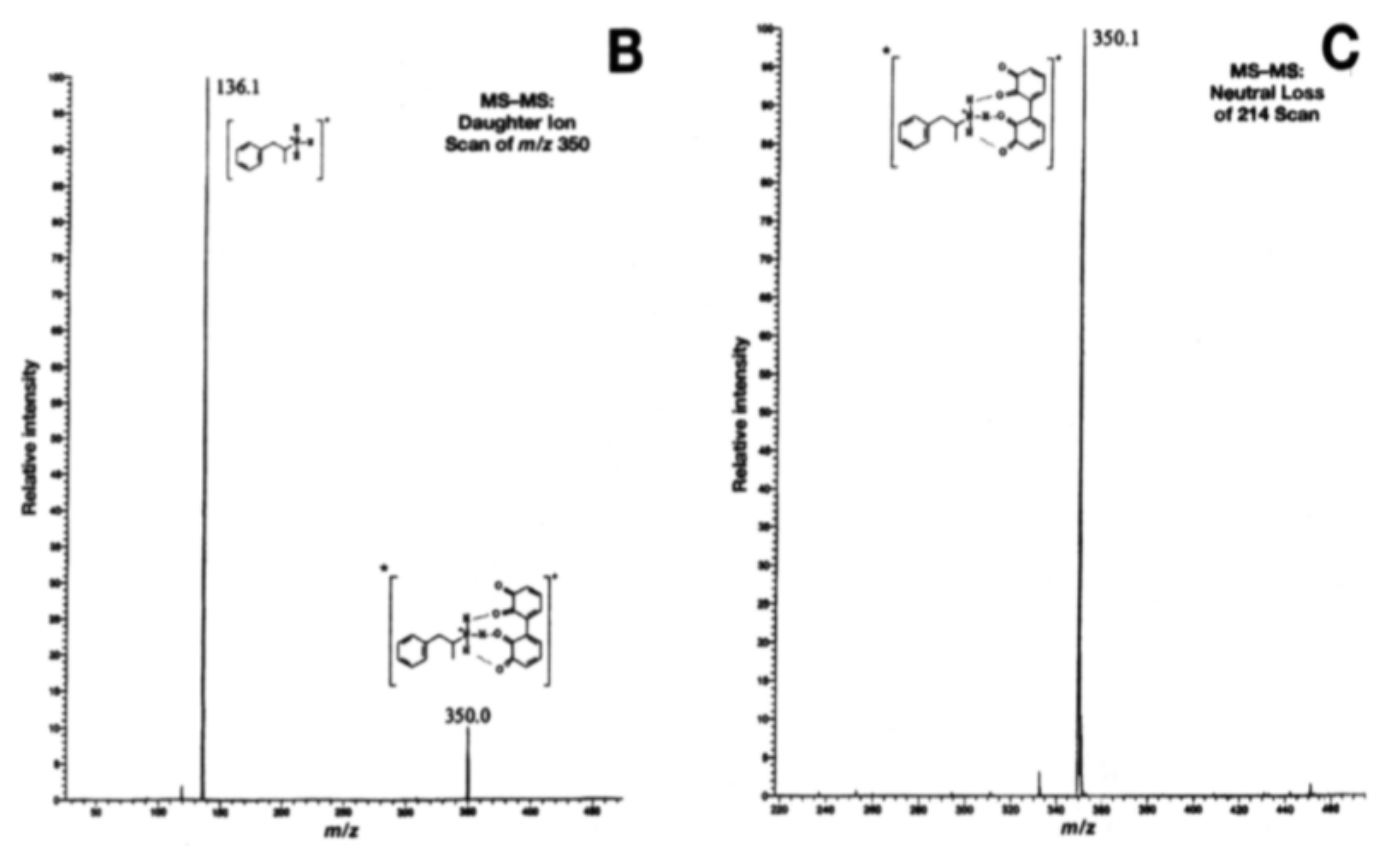

Figure 19: MS spectra of AMP with oxidized catechol. A: Single stage scan of mixture showing the characteristic peak of AMP at $135.9 \mathrm{~m} / \mathrm{z}$ and a peak corresponding to the mass of AMP + catechol at $350.1 \mathrm{~m} / \mathrm{z}$. B: Product ion scan with Q1 fixed on the $\mathrm{m} / \mathrm{z}$ of the potential adduct $(350 \mathrm{~m} / \mathrm{z})$. C: Neutral loss scan for a loss of a $214 \mathrm{~m} / \mathrm{z}$ neutral fragment to account for the mass differential between AMP and the potential adduct. ${ }^{4}$ 
In the present study, drug-melanin associations were studied by UV-Visible spectroscopy, which is an analytical technique that monitors electronic transitions of electrons in a system from the highest occupied molecular orbital (HOMO) to the lowest unoccupied molecular orbital (LUMO) when excited by ultraviolet and/or visible light. When the light energy applied to the system matches the energy required to promote an electron from ground to an excited state, energy is absorbed. The spectrophotometer records the transmittance of light at each wavelength, which is then translated into an absorbance spectrum. The relationship between absorbance and transmittance is described in Equation $1 .{ }^{125}$

$$
A=-\log _{10} T
$$

Equation 2

The electronic transitions (i.e., $\mathrm{n}$ to $\pi^{*}$ and $\pi$ to $\pi^{*}$ ) that are typically recorded are those achievable through irradiation with light ranging from 200-800 nm (ultraviolet and visible light), as seen in Figure 20. It was hypothesized that changes in the UV/Vis spectra of drugs of abuse attributed to the addition of eumelanin would provide insight into the mechanism by which drugs and melanin associate. 


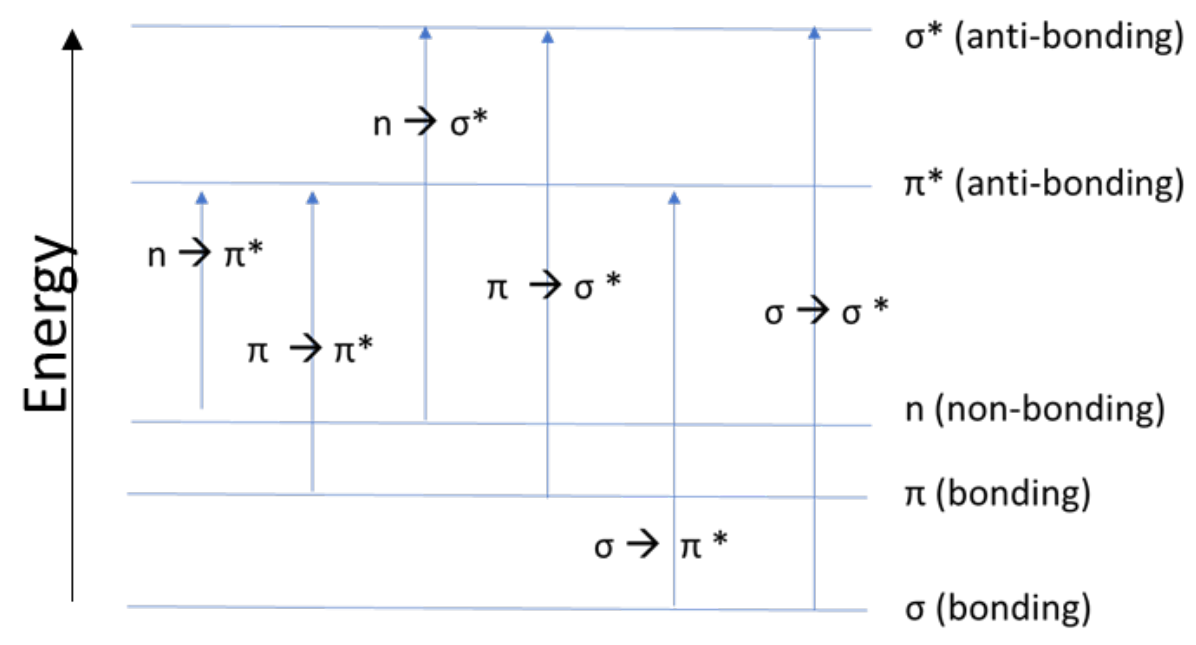

Figure 20: Energy diagram of electronic transitions that may occur in organic molecules. The two lowest energy transitions (i.e., $\mathrm{n} \rightarrow \pi^{*}$ and $\pi \rightarrow \pi^{*}$ ) are the most probable. These transitions are achievable through irradiation with light ranging from $200-800 \mathrm{~nm}$ (ultraviolet and visible light).

6.2 Materials and Methods

Stock solutions of AMP, DZP, MOR, COC were obtained at $1.0 \mathrm{mg} / \mathrm{mL}$ in methanol from Cayman Chemical (Ann Arbor, MI, USA). $\Delta^{9}$-THC was purchased from Cayman Chemical as $10.0 \mathrm{mg} / \mathrm{mL}$ solutions in acetonitrile. These solutions were diluted in methanol (Fisher Scientific; Hampton, NH, USA) to a final concentration of $100 \mathrm{ng} / \mathrm{mL}$ to produce the working solutions for the titrations. A stock solution of $2 \mathrm{mg} / \mathrm{mL}$ of eumelanin (Sigma; St. Louis, MO, USA) was prepared by first dissolving $2 \mathrm{mg}$ of eumelanin in $500 \mu \mathrm{L}$ of DMSO (Fisher Scientific; Hampton NH, USA) and bringing the final volume to $10 \mathrm{~mL}$ with PBS in a volumetric flask.

UV/Visible spectra were recorded on an Agilent Cary 300 UV/Visible spectrophotometer at $37^{\circ} \mathrm{C}$ from $350 \mathrm{~nm}$ to $190 \mathrm{~nm}$ in single beam mode with baseline correction in quartz cuvettes. Solutions of drug at $1 \times 10^{-4} \mathrm{M}$ were prepared in $1 \mathrm{X}$ PBS, $\mathrm{pH} 7.4$, by adding a volume of the $100 \mathrm{ng} / \mathrm{mL}$ stock solution of each analyte. Blank 
solutions used to generate a baseline were prepared by adding a volume of methanol, consistent with the volume of drug solution added to the analytical samples, to 1X PBS, $\mathrm{pH}$ 7.4. The samples were prepared and analyzed in triplicate, and the $\lambda_{\max }$ was recorded with its corresponding absorbance value for each analyte. Next, solutions of drug at $1 \times 10^{-4} \mathrm{M}$ were prepared in $1 \mathrm{X}$ PBS, $\mathrm{pH} 7.4$, with $0,0.3,0.6,0.9,1.2,1.5,1.8,2.1,2.4$, 2.7 , or $3.0 \mu \mathrm{g} / \mathrm{mL}$ eumelanin. Blank solutions used to generate a baseline were prepared by adding a volume of methanol consistent with the volume of drug solution added to the analytical samples and DMSO to achieve a final solution with 5\% DMSO. Nine replicate samples at each concentration of eumelanin were analyzed, and the $\lambda_{\max }$ was recorded with its corresponding absorbance value for each analyte. These values were plotted against the concentration of eumelanin as changes in absorbance at the $\lambda_{\max }$. Data analysis was performed using OriginLab software (Version 2020).

Additional titration curves were generated using this method while changing the $\mathrm{pH}$ and ionic strength of the buffer to begin to probe the mode of association. The $\mathrm{pH}$ of the buffer was adjusted to 5.5 , as to reflect the $\mathrm{pH}$ inside hair matrix cells, ${ }^{13}$ to probe whether the charge state of ionizable functional groups of the drugs played a role in their association with eumelanin. The ionic strength of the buffer was increased 10-fold to disrupt ionic interactions between the drugs and eumelanin to investigate the contribution of ionic interactions in the association between eumelanin and drugs.

\subsection{Results}

The $\lambda_{\max }$ of each analyte was recorded (Table 24), and changes at these wavelengths were observed with the addition of melanin for AMP, COC, and THC. Since the 
absorbance band of the melanin was broadly absorbing with high intensity across the majority of the visible spectrum and exhibited no change with the addition of drugs, the changes in the spectra of the drugs were observed with the addition of melanin. The changes in the presence of melanin were recorded at the $\lambda_{\max }$ of the drug with the addition of the highest concentration of melanin $(3.0 \mu \mathrm{g} / \mathrm{mL})$. For drugs exhibiting more than one transition, changes at the highest energy $\lambda_{\max }\left(\lambda_{\max 1}\right)$ were considered. Addition of eumelanin to solutions of AMP, COC, and THC produced an increase in the absorbace and a red shift at $\lambda_{\max }$, whereas there was no significant change in the absorbance at $\lambda_{\max } 1$ with DZP and MOR, as seen in Figure 21.

Table 24: Recorded $\lambda_{\max }$ values for Drugs of Abuse

\begin{tabular}{|l|r|r|r|}
\hline Drug & $\boldsymbol{\lambda}_{\max \mathbf{1}}$ & $\boldsymbol{\lambda}_{\max \mathbf{2}}$ & $\boldsymbol{\lambda}_{\max \mathbf{3}}$ \\
\hline AMP & 208 & --- & --- \\
\hline COC & 232 & 203 & 274 \\
\hline THC & 209 & --- & --- \\
\hline DZP & 240 & 315 & --- \\
\hline MOR & 215 & 286 & --- \\
\hline
\end{tabular}


A

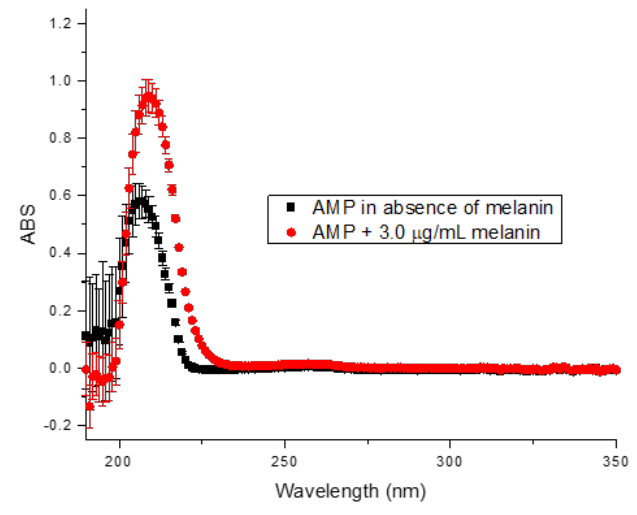

C

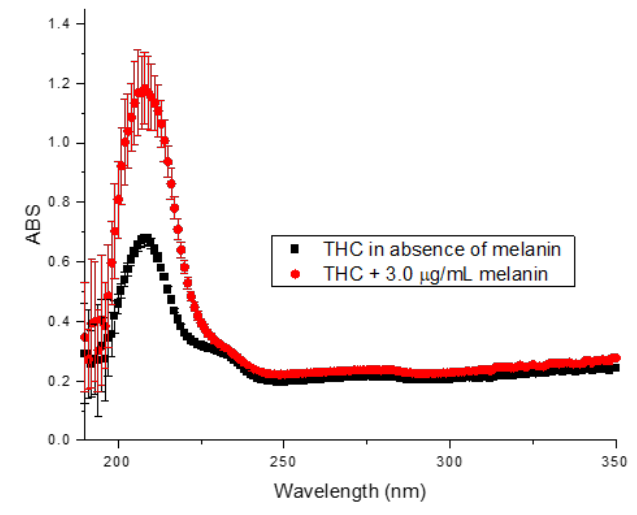

B

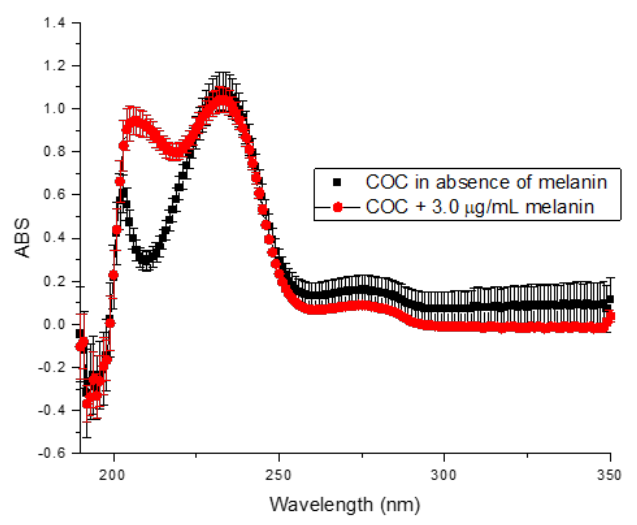

D

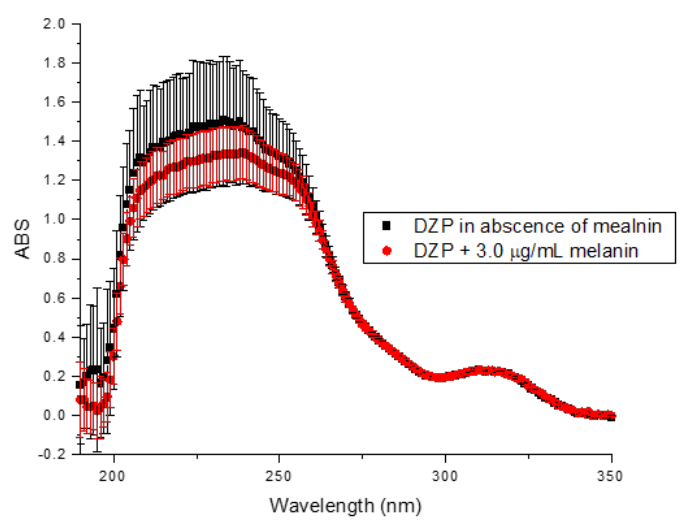

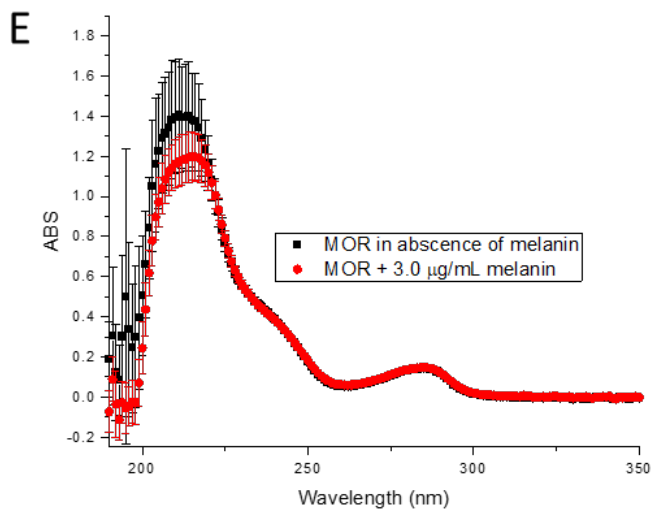

Figure 21: Comparison of spectra for solutions containing $1 \times 10^{-4} \mathrm{M} \mathrm{A}$ : AMP, B: COC, C: THC, D: DZP, E: MOR in the absence of melanin (black) and in the presence of 3.0 $\mu \mathrm{g} / \mathrm{mL}$ melanin (red). Significant increases in the absorbance at the $\lambda_{\max }$ were observed for AMP, COC, and THC in the presence of melanin, while no significant changes were seen in the spectra of MOR and DZP in the presence of melanin when compared with spectra of these drugs in the absence of melanin. 
The titration curves for AMP, COC, and THC were fitted to a linear regression model (Figure 22) to determine if there were a direct correlation between the changes in the spectra of the drugs and the addition of melanin. The linear correlation between the change in absorbance at the $\lambda_{\max }$ and the concentration of melanin was high for AMP and COC, with $\mathrm{R}^{2}$ values of 0.995 and 0.958 , respectively. The correlation between the increase in absorbance at the $\lambda_{\max }$ of THC and the amount of melanin in solution was lower, with an $\mathrm{R}^{2}$ value of 0.827

A

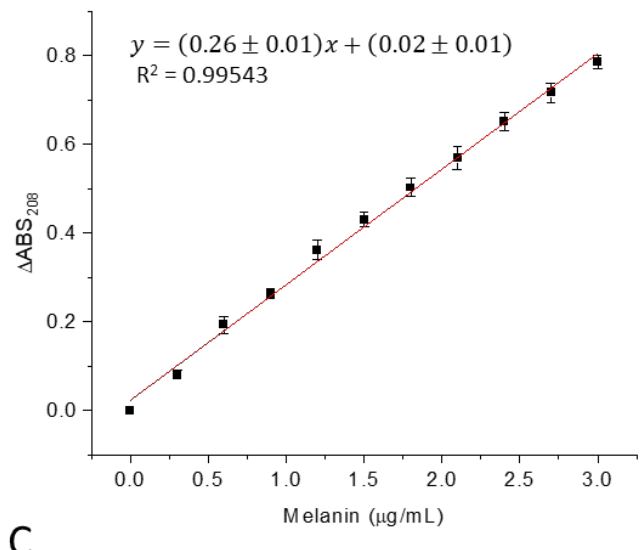

C

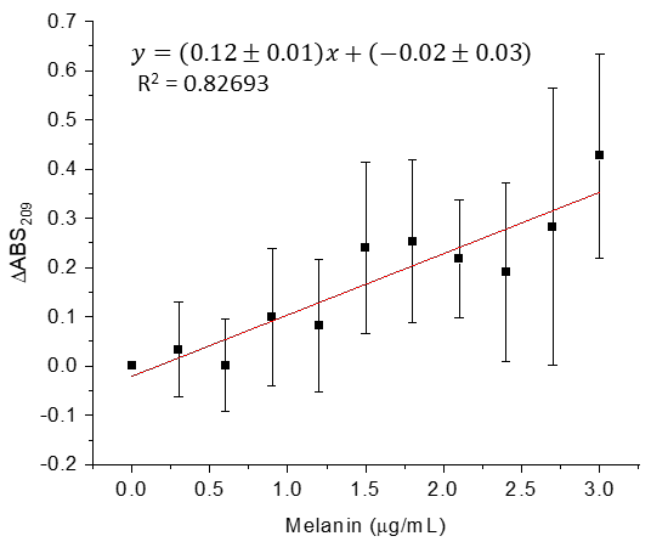

B

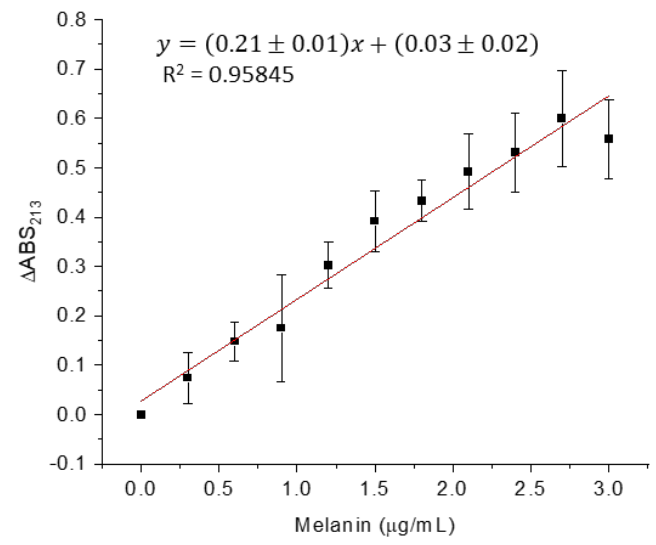

Figure 22: Linear regressions of the change in $\lambda_{\max }$ values for A: AMP, B: COC, C: THC with the addition of eumelanin. 
Titration of melanin into drug solutions under different $\mathrm{pH}$ and ionic strength conditions resulted in differences in the change in intensity of the absorbance at the $\lambda_{\max }$ for both AMP and COC. A Langmuir adsorption model in the form of Equation 3 available in OriginLab (v. 2020) was used to fit the data to determine the point at which the association sites of the melanin were saturated with drug, represented by the inflection point of the Langmuir curve, and the overall change in the absorption at the $\lambda_{\max }$ at the inflection point to understand the intensity of the influence of the electronic structures in melanin on those in the drug molecules.

The formula used in the LangmuirEXT1 in OriginLab (v. 2020) fit function is outlined in Equation 3

$$
y=\frac{a b x^{1-c}}{1+b x^{1-c}}
$$

Equation 3

where $y$ represents $q_{e}, a$ represents $q_{m}, b$ represents $K_{L}$, and $x$ represents $C_{e}$ in the Langmuir equation. The extended model in OriginLab 2020 includes an additional term, $c$, in the exponent, which is to account for the potential of multiple binding sites. ${ }^{126}$ If there is only one binding site, then the equation reduces to the standard Langmuir equation (Equation 4), ehich is expressed such that $C_{e}\left(\mathrm{mg} \cdot \mathrm{g}^{-1}\right)$ is the concentration of the adsorbate at equilibrium, $q_{e}$ is the adsorption capacity at equilibrium, $q_{m}$ is the maximum adsorption capacity, and $K_{L}$ is the Langmuir constant. ${ }^{127}$

$$
q_{e}=\frac{q_{m} K_{L} C_{e}}{1+K_{L} C_{e}}
$$

Equation 4 
A Langmuir model was applied based on the assumption that melanin forms stacks that have a limited number of interaction sites, namely carboxylic acid moieties and $\pi$ systems, on the outer regions of the structures that are exposed in solution for interaction with drugs in solution. Other assumptions of the Langmuir model are that the surface on which the adsorption sites exist is homogeneous, the adsorption sites are equivalent, adsorbed molecules do not interact with one another, all adsorption occurs through the same mechanism, and at maximum adsorption, a monolayer is formed. ${ }^{128}$ Since eumelanin stacks are not a surface in the traditional sense, a monolayer in this system is considered to be when saturation of the binding sites by drug molecules has been achieved since a limited number of drug molecules may interact with the melanin stacks depending on the number of exposed carboxylic acid functions and $\pi$ systems. The Langmuir model can be used to simulate a situation in which the melanin surface has reached a monolayer saturation of drug interacting with the available functions on the exposed regions of the melanin stacks.

Figure 23 shows the increase in the overall intensity of the absorbance at the $\lambda_{\max }$ of AMP at the inflection point of the Langmuir curve at $\mathrm{pH} 5.5(\triangle \mathrm{ABS}=0.45)$ when compared to that at $\mathrm{pH} 7.4(\triangle \mathrm{ABS}=0.23) . \mathrm{At} \mathrm{pH} 5.5$, after a 10 -fold increase in the ionic strength of the PBS buffer, the overall change in intensity of absorbance at the $\lambda_{\max }$ at the inflection point of the Langmuir curve decreased from $\triangle \mathrm{ABS}=0.45$ to $\triangle \mathrm{ABS}=0.39$ (Figure 24). The inflection point at the higher $\mathrm{pH}$ corresponded with a higher concentration of melanin at $10 \mu \mathrm{g} / \mathrm{mL}$ when compared to the lower $\mathrm{pH}$, where the inflection point corresponded with $3 \mu \mathrm{g} / \mathrm{mL}$ of melanin. 


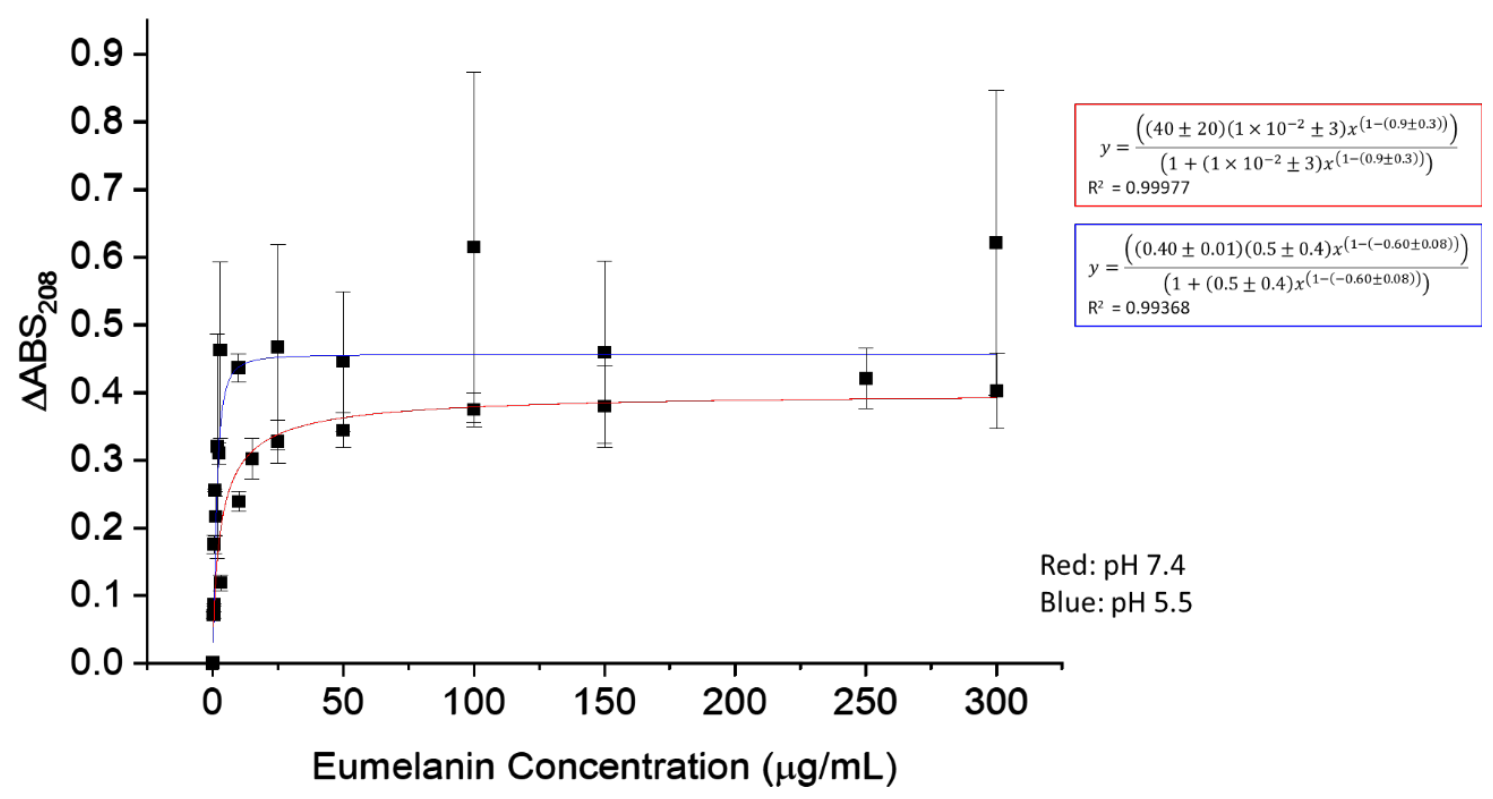

Figure 23: Langmuir fit of change in the intensity of absorbance at $208 \mathrm{~nm}$ with the addition of melanin to solutions with AMP at pH 7.4 (red) and 5.5 (blue).

At $\mathrm{pH} 5.5$, after a 10-fold increase in the ionic strength of the PBS buffer, the overall change in intensity of absorbance at the $\lambda_{\max }$ at the inflection point of the Langmuir curve decreased from $\triangle \mathrm{ABS}=0.45$ to $\triangle \mathrm{ABS}=0.39$ (Figure 24). Also, the inflection point in the $10 \mathrm{X}$ buffer occurred at about $25 \mu \mathrm{g} / \mathrm{mL}$ of melanin, where the inflection point at the lower ionic strength was at $10 \mu \mathrm{g} / \mathrm{mL}$ of melanin. 


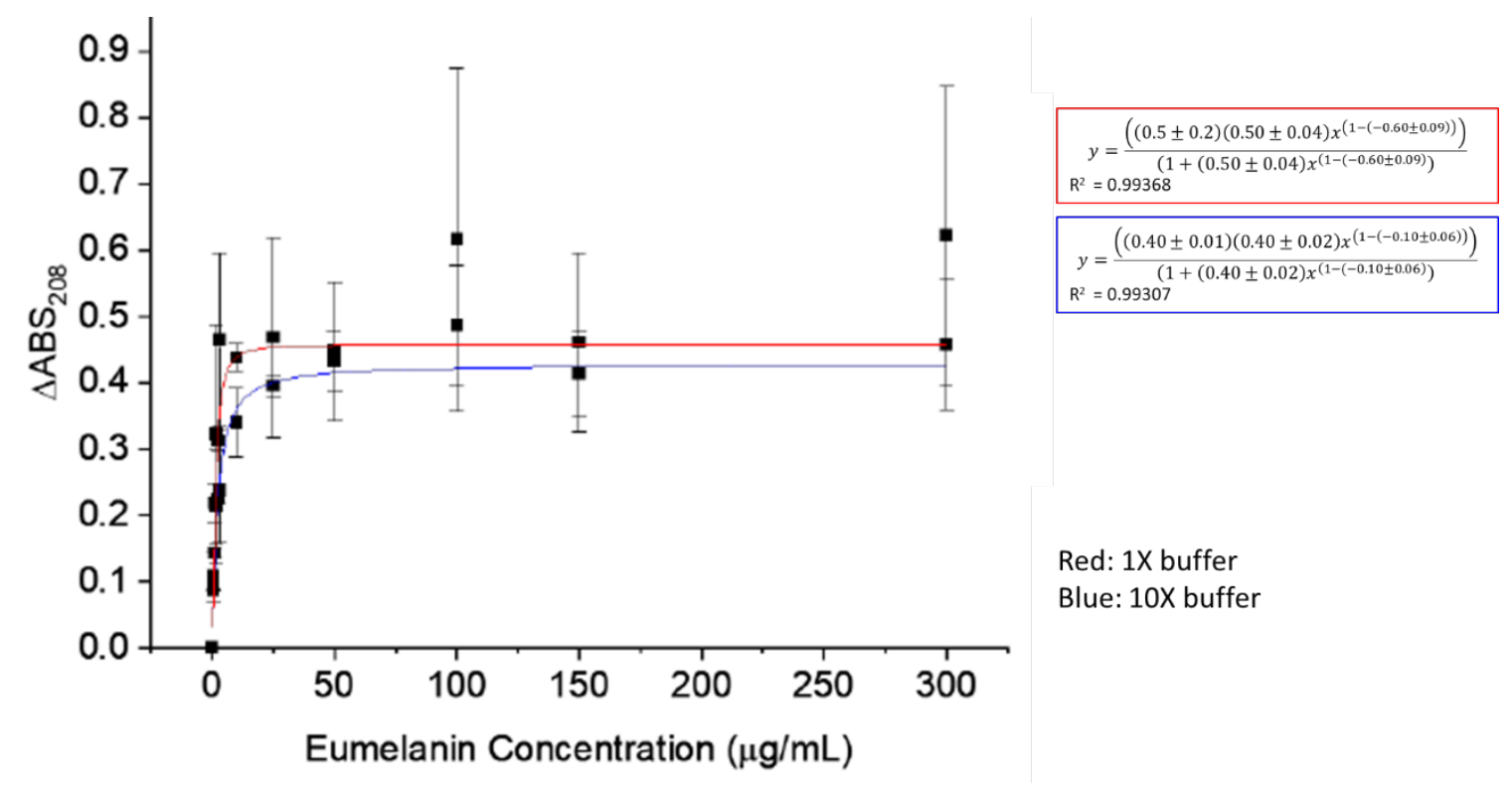

Figure 24: Langmuir fit of change in the intensity of absorbance at $208 \mathrm{~nm}$ with the addition of melanin to solutions with AMP at pH 5.5 in 1X PBS buffer (red) and 10X PBS buffer (blue).

The difference in the change in the absorbance at the inflection point of the Langmuir curve at different $\mathrm{pH}$ values was more dramatic for $\mathrm{COC}$ in the presence of melanin, such that at $\mathrm{pH} 7.4$ the absorbance was 0.78 and at $\mathrm{pH} 5.5$ it was 0.14 (Figure 25). The inflection point of the Langmuir curve occurs at approximately the same concentration of melanin, $10 \mu \mathrm{g} / \mathrm{mL}$, added under both $\mathrm{pH}$ conditions. 


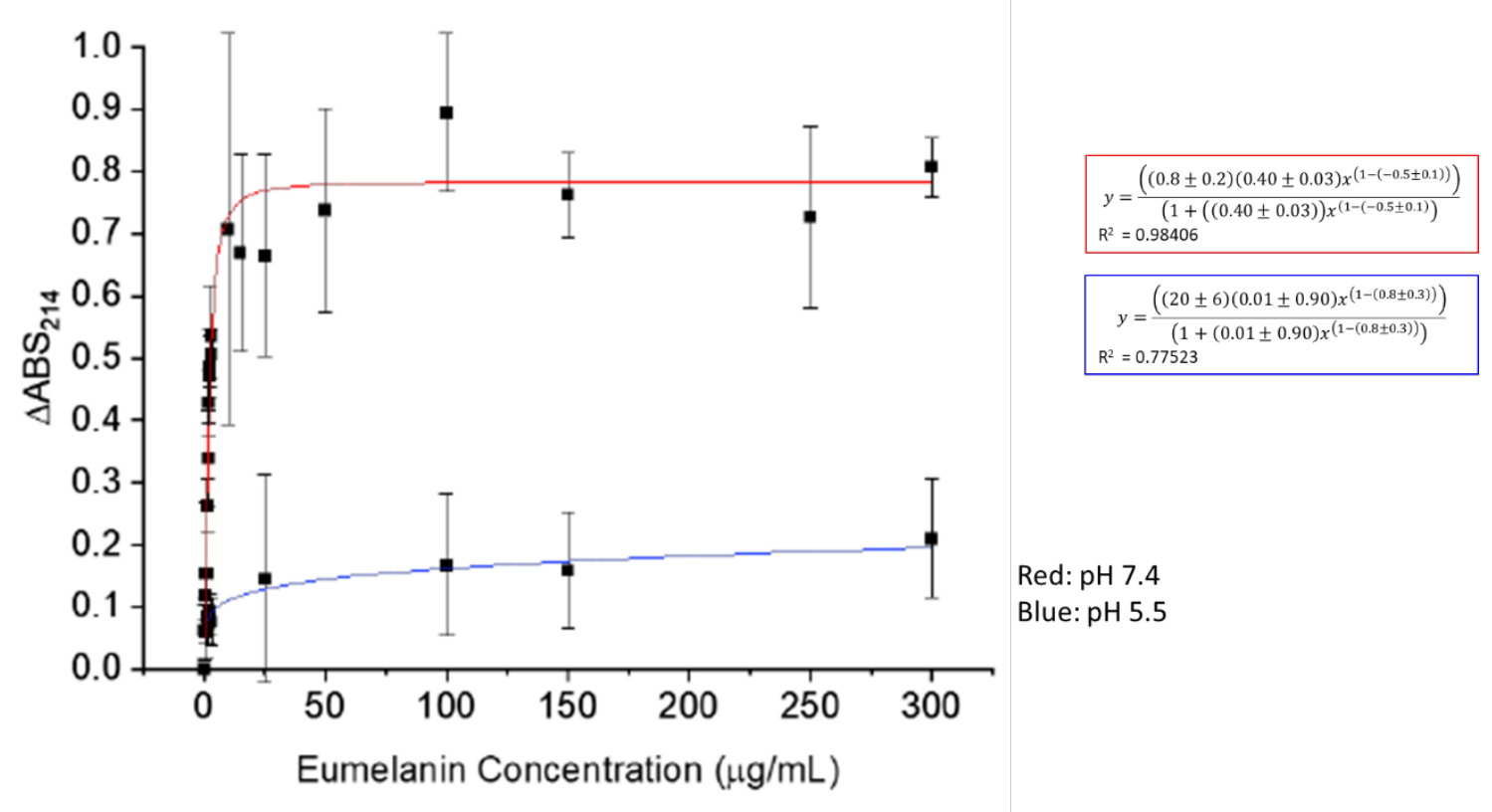

Figure 25: Langmuir fit of change in the intensity of absorbance at the $\lambda_{\max }$ of cocaine with the addition of melanin to solutions with $\mathrm{COC}$ at $\mathrm{pH} 7.4$ (red) and 5.5 (blue).

At $\mathrm{pH} 7.4$, the change in the absorbance at the inflection point of the Langmuir curve was 0.03 at high ionic strength of the buffer, whereas at lower ionic strength the change was 0.78 (Figure 26). The inflection point of the curve occurred at a lower concentration of melanin, $10 \mu \mathrm{g} / \mathrm{mL}$, at the lower ionic strength compared to the $50 \mu \mathrm{g} / \mathrm{mL}$ of melanin at the higher ionic strength. 


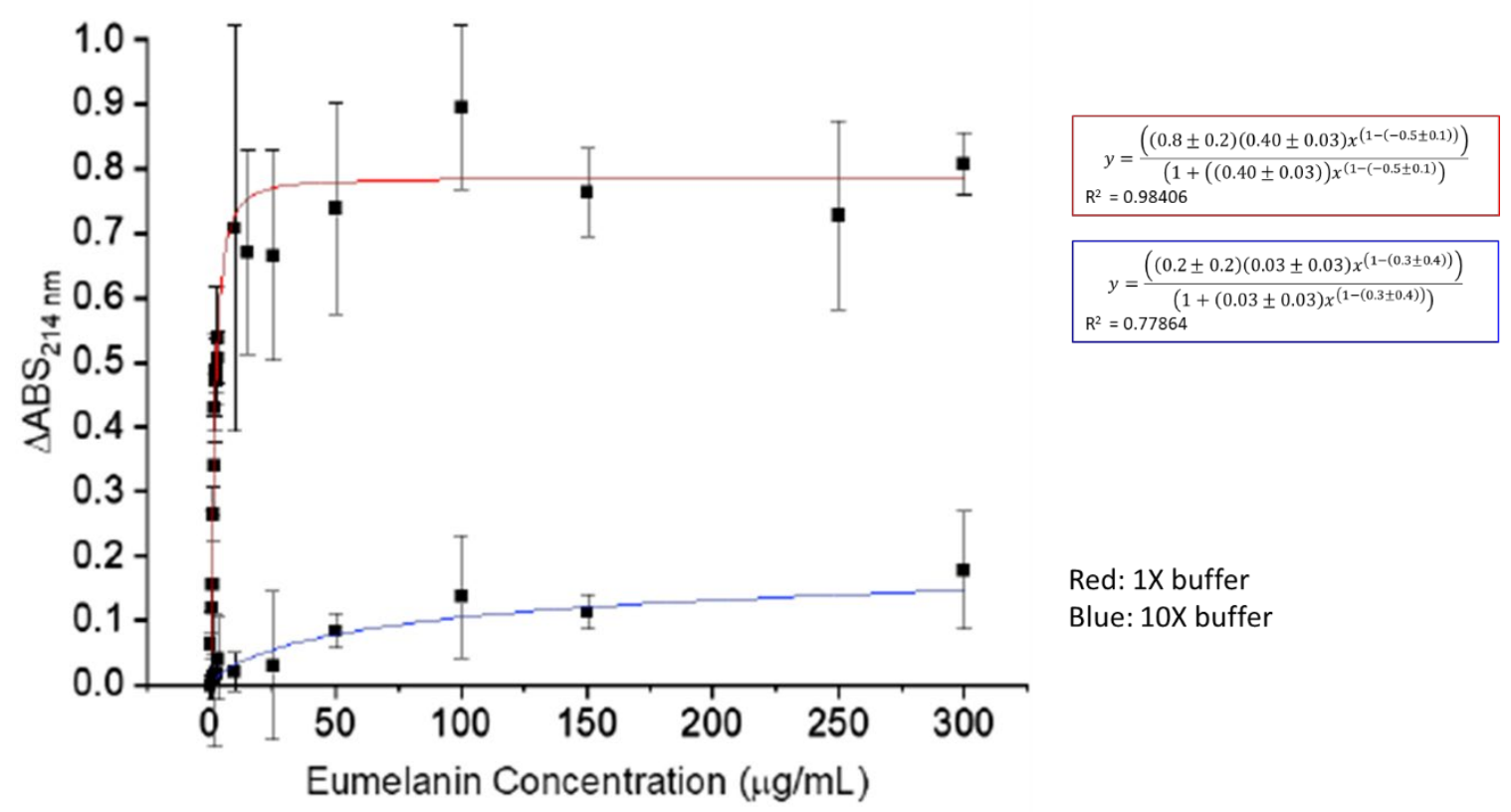

Figure 26: Langmuir fit of change in the intensity of absorbance at $214 \mathrm{~nm}$ with the addition of melanin to solutions with COC at pH 7.4 in $1 X$ PBS buffer (red) and 10X PBS buffer (blue).

The association constant was obtained in Origin 2020 from the Langmuir curves using the value reported for the $b$ parameter of the fit as outlined in Equation 3, and are reported in Table 25. The association constant for AMP was reported for the Langmuir curve generated from AMP and melanin together in a $1 \mathrm{X}$ PBS buffer at $\mathrm{pH} 5.5$, as these conditions were associated with the greatest change in the AMP spectra. The greatest increase in absorbance at the $\lambda_{\max }$ of COC occurred in 1X PBS buffer at $\mathrm{pH} 7.4$; therefore the association constant reported in Table 25 for $\mathrm{COC}$ is from the Langmuir curve resulting from these conditions. The association constant determined for COC with melanin, $1.23 \pm$ $0.2 \times 10^{6}$, was consistent with Shimada et al. who observed an association constant of $8.9 \times 10^{5} \mathrm{M}^{-1}$. The association constant of AMP with melanin, $5.4 \pm 0.4 \times 10^{5} \mathrm{M}^{-1}$, was between the values reported by Simiada et al. and Borges et al. The trend in the three datasets remains the same in that $\mathrm{COC}$ has a higher affinity for melanin than AMP. 
Table 25: Comparison of association constants for eumelanin and drugs.

\begin{tabular}{|r|c|c|c|}
\hline Compound & $\begin{array}{c}\mathbf{K}_{\mathbf{L}}\left(\mathbf{M}^{-\mathbf{1}}\right) \\
\text { from the present } \\
\text { work }\end{array}$ & $\begin{array}{c}\mathbf{K}_{\mathbf{a}} \text { previously reported } \\
\left.\mathbf{( M}^{-1}\right)\end{array}$ & $\begin{array}{c}\mathbf{K}_{\mathbf{a}} \text { previously } \\
\text { reported }\left(\mathbf{M}^{-1}\right)^{4}\end{array}$ \\
\hline AMP & $5.4 \pm 0.4 \times 10^{5}$ & $\begin{array}{c}\text { (+) AMP: } 2.8 \times 10^{5} \\
\text { (-) AMP: } 2.6 \times 10^{5}\end{array}$ & $1.6 \times 10^{6}$ \\
\hline COC & $1.23 \pm 0.2 \times 10^{6}$ & $8.9 \times 10^{5}$ & $4.0 \times 10^{6}$ \\
\hline
\end{tabular}

\subsection{Discussion}

The $\lambda_{\max }$ of benzene is $203.5 \mathrm{~nm}$, and is associated with the conjugated $\pi$ electrons of the system. ${ }^{125}$ When electron-donating functions, such as alkyl chains and more extensive $\pi$-conjugated systems, are covalently bound to benzene a bathochromic shift is observed in the $203.5 \mathrm{~nm}$ absorbance maximum. ${ }^{125}$ Therefore, for each of the analytes in this study, the $\lambda_{\max } 1$ values are likely associated with the $\pi$ electrons in the aromatic rings, as they are all similar to the $\lambda_{\max }$ of benzene. The bathochromic shifts observed in the $\lambda_{\max }{ }_{1}$, as compared to benzene, of each of the analytes is consistent with the more complex structures of the drugs as compared to benzene. The increase in the absorbance of the $\lambda_{\max } 1$ peak in the UV spectra of the tested analytes in the presence of melanin suggests that the interaction between the analytes and melanin is related to van der Waals forces between the $\pi$ systems of the aromatic rings present in each of the analytes and in the structure of eumelanin as has been reported in literature. ${ }^{125}$

It has been theorized that analytes that are cationic at physiological $\mathrm{pH}$ interact with the anionic carboxylic acids of the melanin polymer; the interaction between drugs and melanin can be strengthened by van der Waals forces between aromatic rings in drug molecules and the aromatic indole rings of melanin. ${ }^{23}$ The $\mathrm{pK}_{\mathrm{a}}$ values for AMP and COC 
are 9.9 and 8.6 respectively. While virtually all AMP is cationic under both $\mathrm{pH}$ conditions, $\mathrm{COC}$ is $100 \%$ cationic at $\mathrm{pH} 5.5$ and $>50 \%$ cationic at $\mathrm{pH} 7.4$. It is reasonable to hypothesize an interaction of these cations with the carboxylic acid moieties of eumelanin. The $\mathrm{pK}_{\mathrm{a}}$ of the carboxylic acid moieties is around 5.0; therefore, at $\mathrm{pH} 5$ only a small fraction of the carboxylic acids was anionic and at $\mathrm{pH} 7.4100 \%$ of the carboxylic acids were anionic. As previously suggested, these electrostatic interactions can be envisioned to be stabilized by van der Waals forces between the aromatic $\pi$ systems of the drug molecules and the eumelanin. This explains the bathochromic shift in the $\lambda_{\max }$ of the drug molecules in the presence of melanin. ${ }^{125}$ Evidence of ionic interactions between eumelanin and $\mathrm{COC}$ and AMP is further supported by the fact that the inflection point of the Langmuir curve was associated with greater concentrations of melanin under high ionic strength conditions. This finding suggests that the increased concentration of ions in solution necessitated higher concentrations of melanin for the drugs to saturate the sites for interaction on melanin; thus the buffer salts may have disrupted the ionic interactions between the drugs and melanin.

Since THC lacks an amine function, but was seen to interact with melanin, electrostatic interactions cannot be the only means by which drugs interact with eumelanin. These interactions are likely stabilized by van der Waals forces between the aromatic $\pi$ systems of the drug molecules and the eumelanin, as previously suggested, which is evident from the bathochromic shift in the $\lambda_{\max }$ THC, COC, and AMP in the presence of melanin. ${ }^{125}$

Though correlation was observed between the change in the spectra of THC, AMP, and COC with the addition of melanin, it is important to note that there are factors, other than melanin concentration, that may affect the spectra. Solvent effects may be partially 
responsible for the changes in the spectra. Specifically, when the $\mathrm{pH}$ conditions were changed, the identity of the species in solution were not confirmed, thus the changes in spectra could be a result of the hydrolysis of the species in solution (i.e., ester groups of drug molecules and/or quinones of eumelanin). However, there were changes in the absorbance at the $\lambda_{\max }$ of the drug with increasing concentrations of eumelanin, supporting the conclusion that the species in solution may be interacting.

The differences in the exact values of the association constants is attributed to the two different buffers used in these two studies by Borges et al., who used a formic acid and ammonium formate buffer system in the LC-MS experiments with a $\mathrm{pH}$ of 3.8 and Shimada et al. used a PBS buffer at a $\mathrm{pH}$ of 7.4 with a greater ionic strength that that used by Borges et al. ${ }^{4,16}$ The results reported herein used different $\mathrm{pH}$ and ionic strength conditions to accommodate for the conditions under which each analyte and melanin interacted most significantly. The conditions under which COC were studied were the same in this work as in Shimada et al., which is likely why there is good agreeance between the results. The $\mathrm{pH}$ of the buffer in the present work was adjusted to 5.5 for the study of the interactions between AMP and melanin, which may explain why the affinity was higher in the present study. Additionally, Shimada et al. studied the enantiomers of AMP separately, where a racemic mixture was used in the present work.

\subsection{Conclusions}

The changes in the UV/Vis spectra of THC, COC, and AMP indicated that van der Waals forces and electrostatic interactions likely work in tandem to influence a drug molecule's affinity for melanin. A direct correlation between the addition of melanin to 
solutions of COC and AMP was observed, whereas the correlation was less significant with THC. The structural differences between these drug molecules supports the hypothesis that the van der Waals forces between $\pi$ systems in melanin and drug molecules can allow for associations. These relatively weak forces can be supported by much stronger electrostatic forces, as between charged amines and carboxylic acids, to further strengthen the interaction. Since some drugs, like COC and AMP, interact with melanin more directly than others, like THC, these compounds are more likely to accumulate in hair that has darker brown/black pigment, which results in a bias in hair testing. It is important to follow the present work with additional spectroscopic techniques that allow for a more direct observation of the hypothesized interaction between drug molecules and eumelanin, as UV/Visible spectroscopy is a non-specific technique that is susceptible to solvent effects. Additionally, computational approximations of the interactions are necessary to understand the energy changes in the HOMO and LUMO when the analytes are solvated and mixed with eumelanin. Understanding these associations may allow for changes in the routine analysis of hair to include standardizing the concentrations of drugs known to more strongly associate with eumelanin to the hair's eumelanin content. 


\section{SUMMARY AND PROSPECTUS}

The lack of consensus regarding optimal techniques for the toxicological analysis of hair has resulted in a large literature base from which it is impossible to discern which techniques are the most effective for routine analysis. As toxicological hair analysis can be permissible evidence in court, it is imperative that specific guidelines are formed regarding the workflow necessary for the results and the interpretation of those results to be reliable evidence. Thus, the goals of this work were to investigate the efficacy of a variety of pretreatment parameters for select drugs of abuse to work toward the standardization of forensic toxicological analysis of hair and to investigate the interactions between these analytes and eumelanin.

It has been concluded that decontamination parameters require special attention for analytes with different physical and chemical properties, such that it is impossible to determine a priori which combinations of solvents/solutions, number of consecutive washes, and length of wash steps are necessary to effectively remove surface contamination. Solvent extraction was the most effective technique for extracting a variety of analytes from the hair matrix. Insight into the efficacy of various methodologies may also be achieved by further understanding of the mechanisms by which drugs are held in hair. Spectroscopic studies suggested that interactions between eumelanin and drug molecules are strongest when electrostatic and van der Waals forces are both possible simultaneously, supporting literature suggesting the presence of an ionizable amine in the drug molecule increases its eumelanin affinity because it is capable of interacting with anionic carboxylic acids on eumelanin structures. 
Future work should include similar studies involving the effect of pre-treatment parameters and the association mechanisms regarding metabolites of drugs in hair. Additional work applying DoE methods to authentic HRM and, ultimately, to authentic specimens would help to elucidate if the conclusions made regarding pre-treatment parameters using incorporated/entrapped HRM are applicable to real samples. Additional DoE studies including other parameters known to effect the results of hair analysis, like the use of ultrasonication during extraction, that were not addressed in this work should be studied. Also, additional levels of parameters should be investigated by DoE, for example other decontamination solvents/solutions, such as isopropanol and buffers. Spectroscopic techniques that provide more structural information, such as ${ }^{1} \mathrm{H}$ NMR and mass spectrometry, should be explored to further probe the interactions between drugs and eumelanin. 


\section{REFERENCES}

(1) Barbosa, J.; Faria, J.; Carvalho, F.; Pedro, M.; Queiros, O.; Mroeira, M.; DinisOliveira, R. J. Hair as an alternative matrix in bioanalysis . Bioanalysis. 2016, 8 , 895-914.

(2) Schneider, M. R.; Schmidt-Ullrich, R.; Paus, R. The hair follicle as a dynamic miniorgan. Curr.Biol. 2005, 3, R-132-R-142.

(3) Drug Testing in Hair. CRC Press: Boca Raton, 1996.

(4) Borges, C. R.; Roberts, J. C.; Wilins, D. G.; Rollins, D. E. Cocaine, benzoylecgonine, amphetamine, and $\mathrm{N}$-acetylamphetamine binding to melanin subtypes. J.Anal.Toxicol. 2003, 125, 125-134.

(5) Borges, C. R.; Roberts, J. C.; Wilins, D. G.; Rollins, D. E. Relationship of melanin degradation products to actual melanin content: application to human hair. Anal.Biochem. 2001, 290, 116-125.

(6) Vogliardi, S.; Tucci, M.; Stocchero, G.; Ferrara, S. D.; Favretto, D. Sample preparation methods for determination of drugs of abuse in hair samples: a review. Anal.Chim.Acta. 2015, 1-27.

(7) Kintz, P. Hair Analysis in Forensic Toxicology: An updated review with special focus on pitfalls. Curr.Pharm.Des. 2017, 857, 5480-5486.

(8) Barroso, M.; Gallardo, E.; Vieira, D. N.; Lopez-Rivadulla, M.; Queiroz, J. A. Hair: a complementary source of bioanalytical information in forensic toxicology. Bioanalysis. 2011, 1, 67-79. 
(9) Favretto, D.; Vogliardi, S.; Stocchero, G.; Nalesso, A.; Tucci, M.; Ferrara, S. D. High performance liquid chromatography-high resolution mass spectrometry and micropulverized extraction for the quantification of amphetamines, cocaine, opioids, benzodiazepines, antidepressants and hallucinogens in $2.5 \mathrm{mg}$ hair samples. J.Chromatogr.A. 2011, 38, 6583-6595.

(10) Gryczynski, J.; Schwartz, R. P.; Mitchell, S. G.; O’GRady, K. E.; Ondersma, S. J. Hair drug testing results and self-reported drug use among primary care patients with moderate-risk illicit drug use. DrugAlcoholDepend. 2016, 141, 44-50.

(11) Henderson, G. L. Mechanisms of drug incorporation into hair. ForensicSci.Int. 1993, 63, 19-29.

(12) Aguis, R.; Baumgartner, M. R.; Kintz, P.; Salomone, A.; Tsanaclis, L. European guidelines for workplace drug and alcohol testing in hair. 2015.

(13) Kronstrand, R.; Scott, K. Drug Incorporation into Hair. In Analytical and Practical Aspects of Drug Testing in Hair; Kintz, P., Ed.; CRC: Boca Raton, 2007.

(14) Cuypers, E.; Flanagan, R. J. The interpretation of hair analysis for drugs and drug metabolites. Clin.Toxicol. 2017, 56, 90-100.

(15) Gautman, L.; Scott, K. S.; Cole, M. D. Amphetamine binding to synthetic melanin and scatchard analysis of binding data. J.Anal.Toxicol. 2005, 29, 339-344.

(16) Shimada, K.; Baweja, R.; Sokoloski, T.; Ptail, P. N. Binding characteristics of drugs to synthetic levodopa melanin. J.Pharm.Sci. 1976, 65, 1057-1060. 
(17) Boumba, V. A.; Ziavrou, K. S.; Vougioukalakis, T. Hair as a biological indicator of drug use, drug abuse or chronic exposure to environmental toxicants. Int.J.Toxicol. 2006, 25, 143-163.

(18) Popescu, C.; Hocker, H. Hair - the most sohpisticated biological composite material. Chem.Soc.Rev. 2006, 36, 1282-1291.

(19) Van Scott, E. J. Keratinization and hair growth. Annu.Rev.Med. 1968, 337-350.

(20) Borges, C. R. Roles of Drug Basisity, Melanin Binding, and Cellular Transport in Drug Incorporation into Hair. The University of Utah 2001.

(21) Ito, S.; Wakamatsu, K. Chemistry of mixed melanogenesis - pivotal roles of dopaquinone. Photochem.Photobiol. 2008, 84, 582-592.

(22) Jin, Z.; Fan, H. The modulation of melanin-like materials: methods, characterization and applications. Polym.Int. 2016, 18, 1258-1266.

(23) Kronstrand, R.; Scott, K. S. Drug Incorporation into Hair. Taylor and Francis: Boca Raton 2007, pp 1-23.

(24) Favretto, D.; Vogliardi, S.; Tucci, M.; Simoncello, I.; El Mazloum, R.; Snenghi, R. Occupational exposure to ketamine detected by hair analysis: a retrospective and prospective toxicological study. Forensic Sci.Int. 2016, 265, 193-199.

(25) Kintz, P. Bioanalytical procedures for detection of chemical agents in hair by case of drug-facilitated crimes. Anal.Bioanal.Chem. 2007, 388, 1467-1474. 
(26) Houck, M. Textiles. In Forensic Chemistry Fundamentals and Applications; Siegel, J., Ed.; Wiley: Oxford, 2016; pp 76-115.

(27) Buffoli, B.; Rinaldi, F.; Labanca, M.; Sorbellini, E.; Trink, A.; Guanziroli, E.; Rezzani, R.; Rodella, L. F. The human hair: from anatomy to physiology. Int.J. Dermatol. 2014, 53, 331-341.

(28) Mistry, S.; Chatterjee, M.; Ghosh, J. R.; Chakrabarti, N. K.; Bandyopadhyay, A. R. Variations of scalp, pubic, and auxillary hair. Anthr. Anz. 2011, 69, 117-125.

(29) Grisedale, K. S.; Murphy, G. M.; Brown, H.; Wilson, M. R.; Sinha, S. K. Successful nuclear DNA profiling of rootless hair shafts: a novel approach. Int.J. Leg.Med. 2018, 132, 107-115.

(30) Sachs, H. History of Hair Analysis. ForensicSci.Int. 1997, 84, 7-16.

(31) Baumgartner, A. M.; Jones, P. F.; Baumgartner, W. A.; Black, C. T. Radioimmunoassay of hair for determining opiate abuse histories. J.Nucl.Med. 1979, 20, 748-752.

(32) Valente, D.; Cassini, M.; Pigliapochi, M.; Vansetti, G. Hair as the sample in assessing morphine and cocaine addiction. Clin.Chem. 1981, 27, 1952-1953.

(33) Haley, N. J.; Hoffman, D. Analysis of nicotine and cotinine in hair to determine cigarette smoker status. Clin.Chem. 1985, 31, 1598-1600.

(34) Offidani, C.; Carnevale, A.; Chiarotti, M. Drugs in hair: a new extraction procedure. ForensicSci.Int. 1989, 41, 35-39. 
(35) Harkey, M. R.; Henderson, G. L.; Zhou, C. Simultaneous quantitation of cocaine and its major metabolites in human hair by gas chromatography/mass spectrometry. Anal.Toxicol. 1991, 15, 260-266.

(36) Sachs, H.; Uhl, M. Opiatnachweis in haarextrakten mit hilfe von GC/MS/MS und supercritical fluid extraction (SFE). Toxichem.Krimtech. 1992, 59, 144-148.

(37) Staub, C.; Edder, P.; Veuther, J. L. Importance of supercritical fluid extraction (SFE) in hair analysis. In Drug Testing in Hair; Kintz, P., Ed.; CRC Press: Boca Raton, 1996.

(38) Cooper, G. A. A.; Kronstrand, R.; Kintz, P. Society of Hair Testing guidelines for drug testing in hair. ForensicSci.Int. 2012, 218, 20-24.

(39) Baciu, T.; Borrull, F.; Aguilar, C.; Calull, M. Recent trends in analytical methods and separation techniques for drugs of abuse in hair. Anal.Chim.Acta. 2015, 856, $1-26$.

(40) Breidi, S. E.; Barker, J.; Petroczi, A.; Naughton, D. P. Enzymatic digestion and selective quantification of underivatised delta-9-tetrahydrocannabinol and cocaine in human hair using gas chromatography-mass spectrometry. J.Anal.Methods Chem. 2012, 2012, 1-7.

(41) Sachs, H.; Kintz, P. Testing for drugs in hair critical review of chromatographic procedures since 1992. J.Chromatogr.B. 1998, 21, 147-161. 
(42) Lendoiro, E.; Quintela, O.; de Castro, A.; Cruz, A.; Lopez-Rivadulla, M.;

Concheiro, M. Target screening and confirmation of 35 licit and illicit drugs and metabolites in hair by LC-MSMS. ForensicSci.Int. 2012, 217, 207-215.

(43) Xiang, P.; Shen, M.; Drummer, O. Review: drug concentrations in hair and their relevance in drug facilitated crimes. J.Forensic Legal Med. 2015, 36, 126-135.

(44) Wada, M.; Ikeda, R.; Kuroda, N. Analytical methods for abused drugs in hair and their applications. Anal.Bioanal.Chem. 2010, 397, 1039-1067.

(45) Nakahara, Y. Hair analysis for abused and therepeutic drugs. J.Chromatogr.B. 1999, 733, 161-180.

(46) Ferreira, C.; Paulino, C.; Quintas, A. Extraction procedures for hair forensic toxicological analysis: a mini-review. Chem.Res.Toxicol. 2019, 32, 2367-2381.

(47) Hair Analysis in Clinical and Forensic Toxicology. Academic Press: London, 2015.

(48) Cooper, G. A.; Kronstrand, R.; Kintz, P. Recommendations for hair testing in forensic cases. ForensicSci.Int. 2012, 218, 20-24.

(49) Salomone, A.; Baumgartner, M. R.; Lombardo, T.; Alladio, E.; Di Corcia, D.; Vincenti, M. Effects of various pretreatment procedures on ethyl glucuronide quantification in hair samples: comparion of positivity rates and appraisal of cutoff values. ForensicSci.Int. 2016, 267, 60-65. 
(50) Alladio, E.; Biosa, G.; Seganti, F.; Di Corcia, D.; Salomone, A.; Vincenti, M.; Baumgartner, M. R. Systematic optimization of ethyl glucoronide extraction conditions from scalp hair by design of experiments and its potential effect on cutoff values appraisal. DrugTest.Anal. 2018, 10, 1394-1403.

(51) Broecker, S.; Herre, S.; Pragst, F. General unknown screening in hair by liquid chromatography hybrid quadrupole time-of-flight mass spectrometry (LC-QTOFMS). ForensicSci.Int. 2012, 218, 68-81.

(52) Salomone, A.; Tsanaclis, L.; Aguis, R.; Kintz, P.; Baumgartner, M. R. European guidelines for workplace drug and alcohol testing in hair. DrugTest.Anal. 2016, 8 , 996-1004.

(53) Administration, S. A. and M. H. S. Clinical Drug Testing in Primary Care; Rockville, MD, 2012.

(54) Miki, A.; Katagi, M.; Kamata, T.; Kei, Z.; Tatsuno, M.; ahanishi, T. MALDI-TOF and MALDI-FTICR imaging mass spectrometry of methamphetamine incorporated into hair. J.Mass Spec. 2011, 46, 411-416.

(55) Miki, A.; Katagi, M.; Shima, N.; Kamata, T.; Tatsuno, M.; Nakanishi, T.; Tsuchihashi, H.; Takubo, T.; Suzuki, K. Imaging of methamphetamine incorporated into hair by MALDI-TOF mass spectrometry. ForensicToxicol. 2011, $29,111-116$. 
(56) Porta, T.; Grivet, C.; Kraemer, T.; Varesio, E.; Hopfgartner, G. Single hair cocaine consumption monitoring by mass spectrometric imaging. Anal.Chem. 2011, 83, $4266-4272$.

(57) Shima, N.; Sasaki, K.; Kamata, T.; Matsuta, S.; Katagi, M.; Miki, A.; Zaitsu, K.; Sato, T.; Nakanishi, T.; Tsuchihashi, H.; et al. Single-hair analysis of zolpidem on the supposition of its single administration in drug-facilitated crimes.

ForensicToxicol. 2015, 33, 122-130.

(58) Musshoff, F.; Arrey, T.; Strupat, K. Determination of cocaine, cocaine metabolites and cannabinoids in single hairs by MALDI Fourier transform mass spectrometrypreliminary results. Drug Test.Anal. 2013, 5, 361-365.

(59) Nakanishi, T.; Nirasawa, T.; Takubo, T. Quantitative mass barcode-like image of nicotine in single longitudinally sliced hair sections from long-term smokers by matrix-assisted laser desorption time-of-flight mass spectrometry imaging. J.Anal.Toxicol. 2014, 38, 349-353.

(60) Poetzsch, M.; Steuer, A. E.; Roemmelt, A. T.; Baumgartner, M. R.; Kraemer, T. Single hair analysis of small molecules using MALDI-triple quadrupole MS imaging and LC-MS/MS: investigations on opportunities and pitfalls. Anal.Chem. 2014, 86, 11758-11765.

(61) Shen, M.; Xiang, P.; Shi, Y.; Pu, H.; Yan, H.; Shen, B. Mass imaging of ketamine in a single scalp hair by MALDI-FTMS. Anal.Bioanal.Chem. 2014, 406, 46114616. 
(62) Cuypers, E.; Flinders, B.; Bosman, I. J.; Lusthof, K. J.; VanAsten, A. C.; Tytgat, J.; Heeren, R. M. A. Hydrogen peroxide reactionson cocaine in hair using imaging mass spectrometry. ForensicSci.Int. 2014, 242, 103-110.

(63) Flinders, B.; Cuypers, E.; Zeijlemaker, H.; Tytgat, J.; Heeren, R. M. A. Preparation of longitudinal sections of hair samples for the analysis of cocaine by MALDIMS/MS and TOF-SIMS imaging. DrugTest.Anal. 2015, 7, 859-865.

(64) Kamata, T.; Shima, N.; Sasaki, K.; Matsuta, S.; Takei, S.; Katagi, M.; Miki, A.; Zaitsu, K.; Nakanishi, T.; Sato, T.; et al. Time-course mass spectrometry imaging for depicting drug incorporation into hair. Anal.Chem. 2015, 87, 5476-5481.

(65) Poetzsch, M.; Baumgartner, M. R.; Steuer, A. E.; Kraemer, T. Segmental hair analysis for differentiation of tilidine intake from external contamination using LC-ESI-MS/MS and MALDI-MS/MS imaging. DrugTest.Anal. 2015, 33, $122-$ 130.

(66) Cuypers, E.; Flinders, B.; Boone, C. M.; Bosman, I. J.; Lusthof, K. J.; VanAsten, A. C.; Tytgat, J.; Heeren, R. M. A. Consequences of decontamination procedures in forensic hair analysis using metal-assisted decondary ion mass dpectrometry analysis. Anal.Chem. 2016, 88, 3091-3097.

(67) Tsanaclis, L.; Andraus, M.; Wicks, J. Hair analysis when external contamination is in question: a review of practical approach for the interpretation of results. ForensicSci.Int. 2018, 285, 105-110. 
(68) White, R. M. Drugs in hair. Part I. Metabolism of major drug classes. ForensicSci. Rev. 2017, 29, 23-55.

(69) Stout, P. R.; Ropero-Miller, J. D.; Baylor, R. B.; Mitchell, J. M. External contamination of hair with cocaine: evaluation of external cocaine contamination with development of performance-testing materials. J.Anal.Toxicol. 2006, 30, 490500.

(70) Stout, P. R.; Ropero-Miller, J. D.; Baylor, M. R.; Mitchell, J. M. Morphological changes in human head hair subjected to vairous drug testing decontamination strategies. ForensicSci.Int. 2007, 172, 164-170.

(71) Patzelt, A.; Lademann, J. Drug delivery to hair follicles. ExpertOpin.Drug Delivery. 2013, 10, 787-797.

(72) Cairnes, T.; Hill, V.; Schaffer, M.; Thistle, W. Removing and identifying drug contamination in the analysis of human hair. ForensicSci.Int. 2004, 145, 97-108.

(73) Baumgartner, M. R.; Hill, V. A. Sample preparation techniques. ForensicSci.Int. 1993, 63, 121-135.

(74) Romano, G.; Barbera, N.; Lombardo, I. Hair testing for drugs of abuse: evaluation of external cocaine contamination and risk of false positives. Forensi Sci.Int. 2001, $123,119-129$.

(75) Mantinieks, D.; Wright, P. A systematic investigation of forensic hair decontamination procedures and their limitations. Drug Test.Anal. 2019, 11, 15421555. 
(76) Duvivier, W. F.; Peeters, R. J.; van Beek, T. A.; Nielsen, M. W. F. Evidence based decontamination protocols for the removal of external $\Delta^{9}$-tetrahydrocannabinol (THC) from contaminated hair. Forensic Sci.Int. 2016, 158, 110-118.

(77) Montgomery, M.; LeBeau, M.; Morris-Kukoski, C. New hair testing conclusions. J.Anal.Toxicol. 2017, 41, 161-162.

(78) Davies, C.; Gautam, L.; Grela, A.; Morrissey, J. Variability associated with interpreting drugs within forensic hair analysis: a three-stage interpretation. J.Appl.Toxicol. 2020. https://doi.org/10.1002/jat.3959.

(79) Montgomery, D. C. Design and Analysis of Experiments. Wiley: Danvers 2013.

(80) Oehlert, G. W. A First Course in Design and Analysis of Experiments. Library of Congress 2010.

(81) Mandenius, C.; A, B. Bioprocess optimization using design-of-experiments methodology. Biotechnol.Prog. 2008, 24, 1191-1203.

(82) Salazar, J.; Heinzerling, O.; Müller, R. H.; Möschwitzer, J. P. Process optimization of a novel production method for nanosuspensions using design of experiments (DoE). Int. J. Pharm. 2011, 420, 395-403.

(83) Karakucuk, A.; Celebi, N.; Teksin, Z. S. Preparation of ritonavir nanosuspensions by microfluidization using polymeric stabilizers: I. A design of experiment approach. Eur.J.Pharm. Sci. 2016, 95, 111-121. 
(84) Debrus, B.; Lebrun, P.; Ceccato, A.; Caliaro, G.; Rozet, E.; Nistor, I.; Oprean, R.; Ruperez, F. J.; Barbas, C.; Boulanger, B.; et al. Application of new methodologies based on design of experiments, independent component analysis and design space for robust optimization in liquid chromatography. Anal.Chim.Acta 2011, 391, 3342.

(85) De Backer, B.; Debrus, B.; Lebrun, P.; Theunis, L.; Dubois, N.; Decock, L.; Verstraete, A.; Hubert, P.; Charlier, C. Innovative development and validation of an HPLC/DAD method for the qualitative and quantitative determination of major cannabinoids in cannabis plant material. J.Chromatogr.BAnal.Technol.Biomed. LifeSci. 2009, 877, 4115-4124.

(86) Awotwe-Otoo, D.; Agarabi, C.; Faustino, P. J.; Habib, M. J.; Lee, S.; Khan, M. A.; Shah, R. B. Application of quality by design elements for the development and optimization of an analytical method for protamine sulfate. J.Pharm.Biomed.Anal. 2012, 62, 61-67.

(87) Onjia, A. Chemometric Approach to the Experiment Optimization and Data Evaluation in Analytical Chemistry; Belgrade University: Beograd, 2016.

(88) Mikomangwa, W. P.; Madende, N. A.; Kilonzi, M.; Mlyuka, H. J.; Ndayishimyie, P.; Marealle, A. I.; Mutagonda, R. Unlawful dispensing practice of diazepam: a simulated client approach in community pharmacies in the North-West of Dar-EsSalaam region, Tanzania. BMCHeathServ.Res. 2019, 19, 571-576. 
(89) Johanson, C. E. Assessment of the dependence potential of cocaine in animals. In Cocaine: Pharmacology, Effects, and Treatment of Abuse; Grabowski, J., Ed.; National Institute on Drug Abuse Research Monograph, 1984; pp 54-71.

(90) Stoops, W. W.; Hatton, K. W.; Lofwall, M. R.; Nuzzo, P. A.; Walsh, S. L. Intravenous oxycodone, hydrocodone, and morphine in recreational opiod users: abuse potential and relative potencies. Psychophamacology. 2010, 212, 193-203.

(91) Gardner, E. L. Cannabinoid interaction with brain reward systems - the neurobiological basis of cannabinoid abuse. In Marijuana/Cannabinoids: Neurophysiology and Neurobiology; Murphy, L. L., Bartke, A., Eds.; CRC Press: Boca Raton, 1992; pp 276-335.

(92) Kramer, J. C.; Fischman, V. S.; Littlefield, D. C. Amphetamine abuse: pattern and effects of high doses taken intravenously. J.A.M.A. 1967, 201, 89, 306-309.

(93) Bechtel, L. K.; Holstege, C. P. Criminal poisoning: drug-facilitated sexual assault. Emerg.Med.Clin.N.Am. 2007, 25, 499-525.

(94) Principles of Forensic Toxicology. American Association for Clinical Chemistry: Washington, D.C. 2013.

(95) Karch, S. B.; Drummer, O. Karch’s Pathology of Drugs. CRC Press: Boca Raton 2016.

(96) Ropero-Miller, J. D.; Stout, P. R. Research and Development in Forensic Toxicology Development and Production of Reference Materials for Control and Calibration of Hair Drug Testing, Final Report; Vol. 224523. 
(97) Auerbach, K. Drug Testing Methods. Springer: New York 2007, pp 215-233.

(98) Polettini, A.; Cone, E. J.; Gorelick, D. A.; Huestis, M. A. Incorporation of methamphetamine and amphetamine in human hair following controlled oral methamphetamine administration. Anal.Chim.Acta. 2012, 762,35-43.

(99) Cone, E. J. Mechanisms of drug incorporation into hair. Ther.Drug Monit. 1996, $18,438-443$.

(100) Miguez-Framil, M.; Moreda-Pineiro, A.; Bermejo-Barrera, P.; Lopez, P.; Tabernoero, M. J.; Bermejo, A. M. Improvements on enzymatic hydrolysis of human hair for illicit drug determination by gas chromatography/mass spectrometry. Anal.Chem. 2007, 24, 8564-8570.

(101) Miller, E. I.; Wylie, F. M.; Oliver, J. S. Simultaneous detection and quantification of amphetamines, diazepam and its metabolites, cocaine and its metabolites, and opiates in hair by LC-ESI-MS-MS using a single extraction method. J.Anal.Toxicol. 2018, 32, 457-469.

(102) Netter, F. H. Atals of Human Anatomy. Elsevier: Philedelphia 2006.

(103) Schneider, K. J. Covalent protein adduction by drugs of abuse. Florida International University 2013.

(104) Bragulla, H. H.; Homberger, D. G. Structure and functions of keratin proteins in simple, stratefied, keratinized and cornified epithelia. J.Anat. 2009, 214, 516-559. 
(105) Tsanaclis, L.; Nutt, J.; Bagley, K.; Bevan, S.; Wicks, J. Differentiation between consumption and external contamination when testing for cocaine and cannabis in hair. Drug Test.Anal. 2014, Suppl 1, 37-41.

(106) Morris-Kukoski, C. L.; Montgomery, M. A.; Hammer, R. L. Analysis of extensively washed hair from cocaine users and drug chemists to establish new reporting criteria. J.Anal.Toxicol. 2014, 38, 628-636.

(107) Miyaguchi, H.; Kakuta, M.; Iwata, Y. T.; Matsuda, H.; Tazawa, H.; Kimura, H.; Inoue, $\mathrm{H}$. Development of a micropulverized extraction method for rapid toxicological analysis of methamphetamine in hair. J.Chromatogr.A. 2007, 1163, $43-48$.

(108) Koster, R. A.; Alffenaar, J. W. C.; Greijdanus, B.; VanDerNagel, J. E. L.; Uges, D. R. A. Fast and highly selective LC-MS/MS screening for THC and 16 other abused drugs and metabolites in human hair to monitor patients for drug abuse. Ther.Drug.Monit. 2014, 36, 234-243.

(109) Ropero-Miller, J. D.; Huestis, M. A.; Stout, P. R. Cocaine analytes in human hair: evaluation of concentration ratios in different cocaine sources, drug-user populations and surface-contaminated specimens. J.Anal.Toxicol. 2012, 36, 390398. 
(110) Mueller, A.; Jungen, H.; Iwersen-Bergmann, S.; Raduenz, L.; Lezius, S.; Andersen-Streichert, H. Determination of ethyl glucuronide in human hair samples: a multivariate analysis of the impact of extraction conditions in quantitative results. Forensic Sci.Int. 2016, 271, 43-48.

(111) Duca, R.-C.; Hardy, E.; Salquebre, G.; Appenzeller, B. M. R. Hair decontamination procedure prior to multi-class pesticide analysis. Drug Test.Anal. 2014, Suppl 1, 55-66.

(112) Wang, W. L.; Cone, E. J. Testing human hair for drugs of abuse. IV. Environmental cocaine contamination and washing effects. ForensicSci.Int. 1995, 70, 39-51.

(113) Kintz, P. Interlaboratory comparison of quantitative determinations of drug in hair samples. ForensicSci.Int. 1994, 70, 105-109.

(114) Sroji, K. Testing for drugs in hair - a review of chromatographic procedures. Microchim.Acta. 2006, 713, 191-212.

(115) Chiarotti, M. Overview on extraction procedures. ForensicSci.Int. 1993, 26, 161170.

(116) Vincenti, M.; Salomone, A.; Gerase, E.; Pirro, V. Application of mass spectrometry to hair analyis for forensic toxicologicaliInvestigations. MassSpectrom.Rev. 2013, 32, 312-332. 
(117) Mandry, M. M.; Kraemer, T.; Baumgartner, M. R. Systematic assessment of different solvents for the extraction of drugs of abuse and pharmaceuticals from an authentic hair pool. ForensicSci.Int.2 2018, 282, 137-143.

(118) Potsch, L.; Skopp, G.; Rippin, G. A comparison of ${ }^{3} \mathrm{H}$-cocaine binding on melanin granules and human hair in vitro. Int.J.Legal Med. 1997, 110, 55-62.

(119) Ings, R. M. J. Melanin binding of drugs and its implications. DrugMetab.Rev. 1984, $15,1183-1212$.

(120) Banning, T. P.; Heard, C. M. Binding of doxycycline to keratin, melanin and human epidermal tissue. Int.J.Pharm. 2002, 235, 219-227.

(121) Kronstrand, R.; Forstberg-Peterson, S.; Kagedal, B.; Ahlner, J.; Larson, G. Codeine concentration in hair after oral administration is dependent on melanin content. ClinicalChemistry. 1999, 45, 1485-1494.

(122) Rothe, M.; Pragst, F.; Spiegel, K.; Harrach, T.; Fischer, K.; Kunkel, J. Hair concentrations and self-reported abuse history of 20 amphetamine and ecstasy users. Forensic Sci.Int. 1997, 89, 111-128.

(123) Cappelle, D.; De Keukeleire, S.; Neels, H.; Been, F.; De Doncker, M.; Dom, G.; Crunelle, C. L.; Covaci, A.; van Nuijs, A. L. N. Keratinous matrices for the assessment of drugs of abuse comsumption: a correlation study between hair and nails. DrugTest.Anal. 2018, doi: 10.1002/dta.2356. 
(124) Salazar-Bookaman, M. M.; Fowble, J.; Weber, P.; Patil, P. N. Investigation by NMR spectroscopy of the interaction between synthetic soluble (-)-dopa melanin and drugs. Arch.Pharmacol. 1989, 340, 576-582.

(125) Pavia, D. L.; Lampman, G. M.; Kriz, G. S.; Vyvyan, J. R. Introduction to Spectroscopy. Cengage: Belmont, 2015.

(126) Meroufel, B.; Benali, O.; Benyahia, M.; Benmoussa, Y.; Zenasni, M. A. Adsorptive removal of anionic dye from aqueous solutions by algerian kaolin: characteristics, isotherm, kinetic and thermodynamic studies. J.Mater.Environ. Sci. 2013, 4, 482-491.

(127) Liu, Y. Is the free energy change of adsorption correctly calculated? J.Chem.Eng.Data. 2009, 54, 1981-1985.

(128) Masel, R. I. Principles of Adsorption and Reaction on Solid Surfaces; Wiley: New York, 1996.

(129) Peters, F. T.; Drummer, O.; Musshoff, F. Validation of new methods. ForensicSci.Int. 2007, 165, 216-224.

(130) SWGTOX, X. Scientific Working Group for Forensic Toxicology (SWGTOX) standard practices for method validation in forensic toxicology. J.Anal.Toxicol. 2013, 37, 452-474. 


\section{APPENDICES}

Appendix 1. Biosynthetic pathways for eumelanin and pheomelanin. ${ }^{21}$

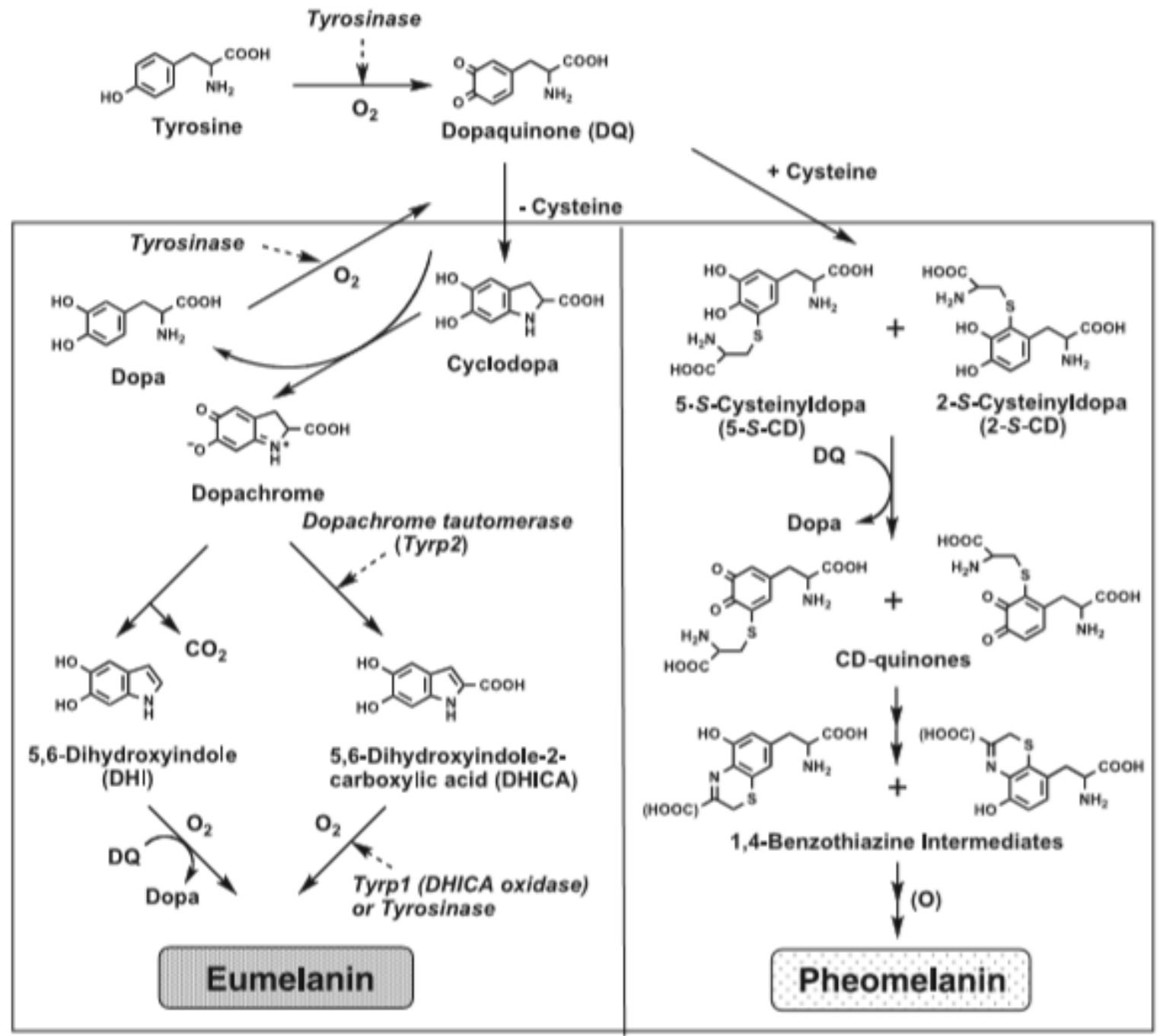


Appendix 2. Analytical method development and validation.

\section{Solid Phase Extraction}

For cleanup of hair extracts, Agilent Bond Elut Certify mixed mode cartridges are used following a method characterized for hair samples by Miller et al. ${ }^{101}$ Determination of the recovery and matrix effects using this SPE method required the preparation of three sets of samples. Set 1 consisted of neat drug, at $100 \mathrm{ng} / \mathrm{mL}$, in methanol. Sets 2 and 3 required the presence of sample matrix. ${ }^{129}$ Thus, sets of $30 \mathrm{mg}$ of blank hair were degraded with each of the extraction techniques previously described. Set 2 was prepared by submitting the degraded matrix to SPE and subsequently spiking the reconstituted solution with drug at $100 \mathrm{ng} / \mathrm{mL}$. Set 3 were spiked with $100 \mathrm{ng} / \mathrm{mL}$ of drug before submitting them to SPE. LCQqQ analysis allowed for the determination of the peak area of the drug. Relationships of the peak areas from the different sample sets were used to characterize the performance of the SPE protocol. The recovery was determined by Equation $5 .{ }^{129}$ Matrix effects were calculated for each extraction technique using Equation $6 .{ }^{129}$

$$
\text { recovery }=\frac{\operatorname{set} 3}{\operatorname{set} 1} \times 100
$$

Equation 5

$$
\text { matrix effects }=\frac{\text { set } 1-\text { set } 2}{\text { set } 2} \times 100
$$

Equation 6 


\section{Accuracy and Precision}

The accuracy and precision measures for amphetamine and diazepam met SWGTOX requirements, as seen in Table 26. ${ }^{130}$ The relative standard deviation was below $20 \%$ for all concentrations of each analyte. The accuracy fell within $\pm 20 \%$ of the spiked concentration for all concentrations of both drugs. 
Table 26: Accuracy and Precision Measures

\begin{tabular}{|c|c|c|c|c|c|c|c|c|}
\hline Compound & & & AMP & DZP & THC & $\mathrm{COC}$ & MOR & HER \\
\hline $\begin{array}{l}\text { Concentration } \\
(\mathrm{ng} / \mathrm{mL})\end{array}$ & $\begin{array}{l}5 \\
(n=5)\end{array}$ & $\begin{array}{l}\text { Standard } \\
\text { Deviation } \\
(\mathrm{ng} / \mathrm{mL})\end{array}$ & 0.3 & 0.9 & 0.1 & 0.4 & 0.03 & 0.3 \\
\hline & & RSD (\%) & 6.5 & 16.6 & 2.1 & 8.0 & 0.63 & 6.0 \\
\hline & & $\begin{array}{l}\text { Accuracy } \\
(\%)\end{array}$ & 98 & 108 & 91 & 97 & 110 & 92 \\
\hline & $\begin{array}{l}75 \\
(n=5)\end{array}$ & $\begin{array}{l}\text { Standard } \\
\text { Deviation } \\
(\mathrm{ng} / \mathrm{mL})\end{array}$ & 1 & 2 & 0.5 & 1 & 0.9 & 0.9 \\
\hline & & RSD (\%) & 2 & 3 & 0.6 & 1 & 1.2 & 1.1 \\
\hline & & $\begin{array}{l}\text { Accuracy } \\
(\%)\end{array}$ & 101 & 98 & 100 & 100 & 99 & 100 \\
\hline & $\begin{array}{l}250 \\
(\mathrm{n}=5)\end{array}$ & $\begin{array}{l}\text { Standard } \\
\text { Deviation } \\
(\mathrm{ng} / \mathrm{mL})\end{array}$ & 5 & 4 & 4 & 3 & 3 & 0.7 \\
\hline & & RSD (\%) & 2 & 2 & 2 & 1 & 1 & 0.3 \\
\hline & & $\begin{array}{l}\text { Accuracy } \\
(\%)\end{array}$ & 100 & 102 & 102 & 99 & 100 & 101 \\
\hline
\end{tabular}




\section{Recovery}

The SPE protocol used was previously applied to a variety of compounds in standard solutions, including amphetamine, diazepam, cocaine, morphine, and the major metabolites of these drugs in hair. Miller et al. observed $99.6 \pm 6.0 \%$ recovery of amphetamine, $98.3 \pm 2.1 \%$ recovery of diazepam, $97.3 \pm 3.1 \%$ recovery of cocaine, and $92.3 \pm 1.1 \%$ recovery of morphine using this method. Table 27 shows the recoveries obtained for these compounds ( $\mathrm{n}=3$ ) and HER and THC using the Bond Elut Certify method used by Miller et al. Recoveries observed were comparable to those previously reported and complied with SWGTOX guidelines of $100 \pm 20 \%$.

Table 27: Recovery of Analytes Using the Bond Elut Certify Method

\begin{tabular}{|c|c|c|}
\hline Compound & $\begin{array}{c}\text { Initial } \\
\text { Concentration } \\
(\mathrm{ng} / \mathrm{mL})\end{array}$ & \% Recovery \\
\hline AMP & \multirow{6}{*}{100} & $98 \pm 2$ \\
\hline DZP & & $94 \pm 2$ \\
\hline THC & & $111 \pm 19$ \\
\hline $\mathrm{COC}$ & & $98 \pm 12$ \\
\hline MOR & & $94 \pm 15$ \\
\hline HER & & $93 \pm 13$ \\
\hline
\end{tabular}




\section{Matrix Effects}

Matrix effects obtained, Table 28, were comparable to those reported by Miller et al. and complied with SWGTOX guidelines. The matrix effects were reported as $96.4 \pm 13.7 \%$ for COC, $85.4 \pm 11.1 \%$ for MOR, $95.5 \pm 13.9 \%$ for DZP, $97.9 \pm 3.4 \%$ for AMP.

Table 28: Matrix effects observed for each analyte under enzymatic degradation and solvent extraction conditions

\begin{tabular}{|l|c|r|r|r|r|r|}
\hline Extraction Technique & AMP & DZP & THC & COC & MOR & HER \\
\hline Enzymatic Degradation & $105 \%$ & $97 \%$ & $111 \%$ & $97 \%$ & $88 \%$ & $89 \%$ \\
\hline Solvent Extraction & $111 \%$ & $94 \%$ & $119 \%$ & $92 \%$ & $82 \%$ & $83 \%$ \\
\hline
\end{tabular}

\section{HPLC -QqQ Analysis}

For drug analysis, $1 \mu \mathrm{L}$ of each isolate was injected onto a reversed-phase liquid chromatography column and subsequently analyzed by QqQ-MS/MS. The compounds were separated using a $1.8 \mu \mathrm{m}$ Zorbax Eclipse Plus $\mathrm{C}_{18}$ rapid resolution HD column $(2.1 \mathrm{x}$ $150 \mathrm{~mm}$ ) from Agilent. The aqueous mobile phase used was water with $0.1 \%$ formic acid and $5 \mathrm{mM}$ ammonium formate. The organic phase was $\mathrm{MeOH}$ with $0.1 \%$ formic acid. The separation was conducted using a gradient solvent system such that the relative percentages of the aqueous and organic phases varied over the time of analysis. The gradient was as follows: at 0 min the mobile phase was a $95 \%$ aqueous and $5 \%$ organic mixture, from 0 to 5 min the gradient adjusted to $100 \%$ organic mobile phase, from 5 to 8 min the gradient was held at $100 \%$ organic to flush the column. The mobile phase flow rate was $0.3 \mathrm{~mL} / \mathrm{min}$. Gradient elution resulted in a LC-QqQ method that allowed for baseline resolution of the compounds of interest. 
An LC-QqQ-MS multiple reaction monitoring (MRM) method was used for the identification of analytes. The peaks were confirmed by MRM transitions corresponding to the integrated peak. MRM peaks were specified in the LC-MS method based on the two most abundant transitions, one as a quantifier and the other as a qualifier. These transitions were identified by Agilent's MassHunter Optimizer program and are listed in Table 29. The data was acquired in Agilent MassHunter Qualitative software. The spectral peak areas were integrated, and the data files were then uploaded in to the MassHunter Quantitative software. 
Table 29: MRM Transitions Selected for $L C-Q q Q$ Method

\begin{tabular}{|c|c|c|}
\hline \multirow[t]{2}{*}{ Compound Name } & \multicolumn{2}{|c|}{ MRM Transition(s) } \\
\hline & Precursor Ion (m/z) & Product Ion $(\mathrm{m} / \mathrm{z})$ \\
\hline \multirow[t]{2}{*}{ AMP } & 136 & 119 \\
\hline & & 91 \\
\hline AMP-D11 & 147 & 98 \\
\hline \multirow[t]{2}{*}{$\mathrm{COC}$} & 304 & 182 \\
\hline & & 82 \\
\hline COC-D3 & 307 & 185 \\
\hline \multirow[t]{2}{*}{ DZP } & 285 & 222 \\
\hline & & 193 \\
\hline DZP-D5 & 290 & 193 \\
\hline \multirow[t]{2}{*}{ MOR } & 286 & 201 \\
\hline & & 155 \\
\hline MOR-D3 & 289 & 126 \\
\hline \multirow[t]{2}{*}{ HER } & 370 & 211 \\
\hline & & 165 \\
\hline \multirow[t]{2}{*}{ THC } & 315 & 193 \\
\hline & & 123 \\
\hline THC-D3 & 318 & 93 \\
\hline
\end{tabular}


A calibration curve was generated using drug standards purchased from Cerilliant with a $1 \mathrm{mg} / \mathrm{mL}$ concentration and the corresponding deuterated compound. The drug standards were diluted in $\mathrm{MeOH}$ to a final volume of $500 \mu \mathrm{L}$ to generate calibration solutions with $250,200,150,75,35,15,5$, and $1 \mathrm{ng} / \mathrm{mL}$ concentrations. The calibrators also had a concentration of $100 \mathrm{ng} / \mathrm{mL}$ of deuterated internal standard.

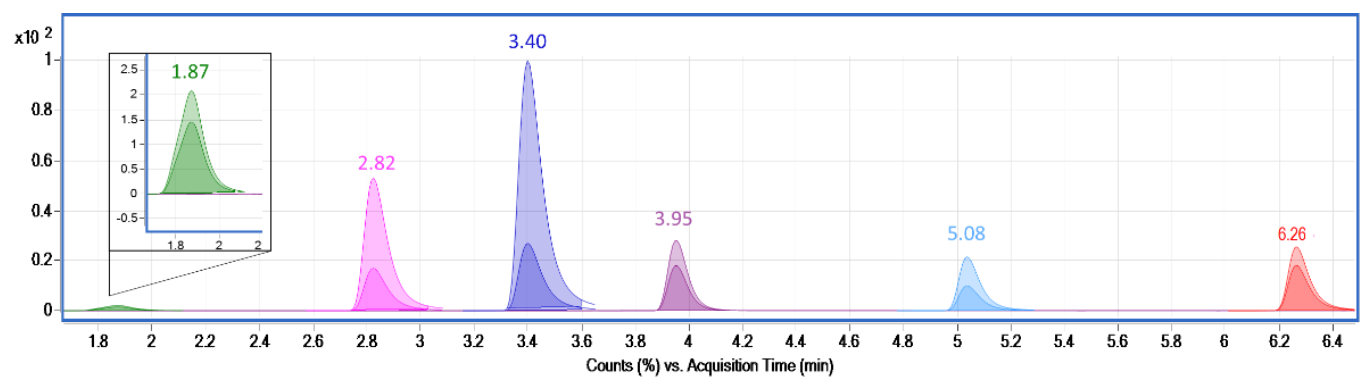

Figure 27: LC separation (from left to right) of morphine, amphetamine, cocaine, heroin, diazepam, and 49-tetrahydrocannabinol at $75 \mathrm{ng} / \mathrm{mL}$. The MRM spectrum of the six separated substances, showing two unique transitions for each and labeled with their respective retention times. 
VITA

JENNETT CHENEVERT AIJALA

\begin{tabular}{|c|c|}
\hline \multirow[t]{3}{*}{$2011-2015$} & B.S., Chemistry \\
\hline & Simmons University \\
\hline & Boston, MA \\
\hline \multirow[t]{3}{*}{$2011-2015$} & AmeriCorps Corps Member \\
\hline & Jumpstart \\
\hline & Boston, MA \\
\hline \multirow[t]{3}{*}{2012} & W.M. Keck Surf Summer Intern \\
\hline & Simmons University \\
\hline & Boston, MA \\
\hline \multirow[t]{3}{*}{$2013-2015$} & Summer Research Intern \\
\hline & Abbvie Bioresearch Center \\
\hline & Worcester, MA \\
\hline \multirow[t]{3}{*}{$2015-2017$} & Teaching Assistant \\
\hline & Florida International University \\
\hline & Miami, Florida \\
\hline \multirow[t]{3}{*}{ 2015-2018 } & M.S., Chemistry \\
\hline & Florida International University \\
\hline & Miami, Florida \\
\hline \multirow[t]{3}{*}{$2017-2020$} & National Institute of Justice Graduate Research Fellow \\
\hline & Florida International University \\
\hline & Miami, Florida \\
\hline \multirow[t]{3}{*}{$2018-2020$} & Doctoral Candidate \\
\hline & Florida International University \\
\hline & Miami, Florida \\
\hline
\end{tabular}




\section{PUBLICATIONS AND PRESENTATIONS}

Chenevert, J.M.; DeCaprio, A.P. (2018). Systematic Comparison of Analytical Parameters in Forensic Hair Analysis using Statistical Design of Experiments (DoE). Pittsburgh Conference on Analytical Chemistry and Applied Spectroscopy, Orlando, FL; February 26-March 1.

Chenevert, J.M; Kimble, A.N.; DeCaprio, A.P. (2018). Assessment of Incorporation of Amphetamine and Diazepam into Human Head Hair for Preparation of Hair Reference Material. American Academy of Forensic Sciences, Seattle, WA; February 19-24.

Aijala, J.C.; DeCaprio, A.P. (2019). Application of Statistical Design of Experiments (DoE) to Assess External Decontamination Methods in Forensic Hair Analysis. American Academy of Forensic Sciences, Baltimore, MD; February 18-22.

Aijala, J.C.; DeCaprio, A.P. (2019). Assessing External Decontamination and Extraction Parameters by Statistical Design of Experiments for Forensic Hair Analysis. Pittsburgh Conference on Analytical Chemistry and Applied Spectroscopy, Philadelphia, PA; March $16-21$.

Aijala, J.C.; DeCaprio, A.P. (2019) Systematic Comparison of Decontamination Parameters Associated with the Toxicological Analysis of Human Head Hair. Society of Forensic Toxicologists, San Anotnio, TX; October 13-18.

Aijala, J.C.; Wu, W.; DeCaprio, A.P. Application of Statistical Design of Experiments to Assess Pre-treatment Parameters in Forensic Toxicological Hair Analysis. Forensic Chemistry, 2020, In Review.

Aijala, J.C.; Wu, W.; DeCaprio, A.P. Assessing Hair Decontamination Protocols for

Diazepam, Heroin, Cocaine, and $\Delta^{9}$-Tetrahydrocannabinol by Statistical Design of Experiments. Journal of Analytical Toxicology, 2020. In Review. 